\title{
Poster Presentations Abstracts, 23rd Annual Meeting of the International Association of Medical Science Educators, Roanoke, VA, USA, June 8-11, 2019
}

Published online: 12 December 2019

(C) International Association of Medical Science Educators 2019

\section{ASSESSMENT}

Poster: 100

\section{Quantifiable Predictors of Step 2 CS Success}

Vincent J. Sinatra, Milana Sapozhnikov, Jennifer Grossman, Bonnie Granat

${ }^{1}$ Downstate College of Medicine, State University of New York, New York, USA

PURPOSE Previous literature has focused on predictors of success on USMLE Step 1 and Step 2 Clinical Knowledge (CK). While Step 2 Clinical Skills (CS) remains a mainstay for standardized evaluation of clinical skills, determinants of success on Step 2 CS have not yet been elucidated. We set out to (1) identify quantifiable factors that can predict future performance on Step 2 CS and (2) determine if a medical school curriculum that is competency-based with multi-modal clinical skills assessments provides predictors of students who are at risk of failing Step 2 CS

METHODS Three hundred forty-two de-identified SUNY Downstate medical students' scores from pre-clinical, clerkship, and standardized examinations were collected: CS Multiple Choice exams, OSCEs, NBME Clerkship Shelf exams, undergraduate GPA, MCAT, Step 1, Step 2 CK and Step 2 CS results. Students were divided into two groups based on Step 2 CS performance: Pass $(n=326)$ and Fail $(n=16)$. An independent two-tailed $t$ test was performed to compare the two groups. Significant relationships were those determined to have a $p$ value of $<$ 0.05 .

RESULTS There was a significant difference in performance between the 2 groups on all shelf exams as well as Step 1 and Step 2 CK: medicine $(p=0.001)$, surgery $(p=0.007)$, ob $/$ gyn $(p=$ $0.027)$, pediatrics $(p=0.003)$, psychiatry $(p=0.014)$, neurology $(p=0.033)$, Step $1(p=0.006)$, and Step 2 CK $(p=0.001)$. The Pass group performed better on all OSCE exams, preclinical CSMC exams, and undergraduate GPA; however, these results did not reach statistical significance.

CONCLUSION Those who passed Step 2 CS received significantly higher scores throughout all core clerkships with an association found between better performance on OSCE exams and receiving a passing score on Step 2 CS. Focusing on early intervention for consistently lower shelf performers may improve clinical skills deficits in those at risk of failing Step 2 CS.

\section{ASSESSMENT}

Poster: 101

The Utilization of Peer Feedback During Collaborative Learning in Medical Education: a Systematic Review

Sarah Lerchenfeldt ${ }^{1},{\text { Misa } \mathrm{Mi}^{1} \text {, Marty Eng }}^{2}$

${ }^{1}$ Department of Foundational Medical Studies, Oakland University William Beaumont School of Medicine, Rochester, MI, 48309 USA ${ }^{2}$ Department of Pharmacy Practice, Cedarville University, Cedarville, $\mathrm{OH}$ 45314 USA

PURPOSE Peer evaluation can provide valuable feedback to medical students, and increase student confidence as well as the quality of work. The goal of this systematic review is to examine the utilization, effectiveness, and quality of peer feedback in a collaborative learning environment, specifically in medical education.

METHODS The PRISMA statement for reporting in systematic reviews and meta-analysis was used to guide the process of conducting the systematic review. Evaluation of level of evidence (Colbert) and types of outcomes (Kirkpatrick) were used. Two main authors reviewed articles with a third deciding on conflicting results.

RESULTS The final review included 31 studies. The majority of studies $(n=27)$ examined student peer feedback evaluation in a classroom setting; other settings included clinical and OSCE environments. Peer feedback was evaluated through collaborative learning activities integrated into preclinical courses $(n=7)$ and clerkships $(n=2)$, although many studies lacked clear information on where peer feedback was evaluated in the curriculum. Problem-based learning and team-based learning were the most common collaborative learning settings. Eleven studies reported that students received instruction on how to provide appropriate peer feedback. Seventeen studies evaluated the effect of peer feedback on professionalism; 12 of those studies evaluated peer feedback effectiveness for assessing professionalism and seven evaluated the peer feedback use for professional behavior development. Ten studies examined the effect of peer feedback on student learning. In addition, there were six studies that examined the role of peer feedback on team dynamics.

CONCLUSIONS This systematic review indicates that peer feedback in a collaborative learning environment may be a reliable assessment for professionalism and may aid in the development of professional behavior. The review suggests implications for further research on the impact of 
peer feedback, including the effectiveness of providing instruction on how to provide appropriate peer feedback.

\section{ASSESSMENT}

Poster: 102

\section{An Innovative Self-Directed Video-Based Course to Improve Medical Students Note Writing}

Sarah Scott, Valeriy Kozmenko, Valerie Hearns, Brian Wallenburg

${ }^{1}$ Sanford School of Medicine, University of South Dakota, Vermillion, USA

PURPOSE The high-stakes Objective Structured Clinical Examination (OSCE) is a required South Dakota Sanford School of Medicine (USD SSOM) test that medical students need to pass at the end of Pillar 2. It closely represents the United States Medical License Examination STEP 2 Clinical Skills (USMLE STEP 2 CS) national board exam. The OSCE consists of a series of standardized patient (SP) encounters, followed by writing a patient note. In the past, approximately $75 \%$ of Pillar 2 students needed to remediate the note-writing component. Nationwide, there is a trend in increasing failure rates in the note-writing part of the USMLE STEP 2 CS exam. USD SSOM developed an Enhanced Patient Note Writer (EPNW) program for students to practice writing notes, but it has not improved students note writing skills tested at the OSCE. We hypothesize supplementing the EPNW with individualized feedback to the students regarding their note writing skills will improve their performance during the high-stakes OSCE.

METHODS A collection of video cases was created showing an interaction between a student doctor and SP. Medical students will watch a video, get the history and physical exam information, and then use EPNW to write a patient note within $10 \mathrm{~min}$, the time constraint as per USMLE STEP 2 CS requirements. After completing a note, a note-grading checklist will become available. Students will self-grade their notes, and the scores will be saved on the server. Researchers will monitor students' progress during three months of preparation for the OSCE.

RESULTS We expect to see students note-writing scores improve due to the individualized feedback provided by the software.

CONCLUSION Creation of an automated system that provides individualized feedback to medical students regarding their note writing skills may improve their performance in handling patient documentation and offset the high cost if SPs and faculty were used instead.

\section{ASSESSMENT}

Poster: 103

Even Flow: Determining Characteristics of Effective Teamwork in the Operating Room with Focus Group Interviews

Deborah D. Garbee ${ }^{1}$, Laura S. Bonanno ${ }^{1}$, Kathryn E. Kerdolff ${ }^{2}$, John T. Paige $^{2}$

${ }^{1}$ School of Nursing in New Orleans, Louisiana State University Health Sciences Center, New Orleans, USA

${ }^{2}$ School of Medicine in New Orleans, Louisiana State University Health Sciences Center, New Orleans, USA

PURPOSE Effective teamwork is essential for safe, quality care in the operating room (OR). In practice, however, OR team function is often hampered by differing perceptions of what constitutes effective teamwork among the various professions. We investigated whether common ground exists among OR personnel regarding the importance of certain teambased competencies.

METHODS Semi-structured focus group interviews were conducted involving the various members of an interprofessional OR team (surgeons, anesthesiologists, nurse anesthetists, surgical technicians, and circulating nurses). For each focus group, participants were asked questions related to teamwork and team function in the OR. Responses were digitally recorded and transcribed. Qualitative analysis was undertaken by two reviewers who identified major themes related to effective teamwork. Intercoder agreement was employed to confirm findings and themes. Data collection continued until data saturation was obtained.

RESULTS Three focus groups involving 14 individuals (2 surgeons, 1 anesthesiologist, 8 nurse anesthetists, 2 circulating nurses and 1 surgical technician) were conducted over a 1-month period in 2017. Four major themes related to effective teamwork emerged from analysis: (1) Smooth flow (2) United effort (3) Communication and (4) Positive attitude.

CONCLUSION Among the various professions in the OR, agreement regarding effective teamwork centers around the concepts of procedural flow unified effort, clear communication, and positive attitude of the team. These findings will be used to help design a quick assessment tool for formative debriefing in the OR.

\section{ASSESSMENT}

Poster: 104

One Step ForWard and NO Steps Back! NBME Subject Exam Scores and Transition to an Integrated Clinical Curriculum

Kirstin Nackers, Raquel Tatar, Eileen Cowan, Laura Zakowski, Katharina Stewart, Sarah Ahrens, David Tillman, Laura Jacques, Shobhina Chheda

${ }^{1}$ SMPHUW-Madison, Madison, USA

Award Nominee

PURPOSE We used NBME subject exam scores and pass rates to study how moving from a traditional clerkship model to integrated clinical blocks affected student acquisition of medical knowledge. Recent AAMC data shows that two-thirds of medical schools are currently undergoing/planning substantial curriculum changes. (1) During periods of curricular change, it is desirable to track assessment outcomes to inform leadership of possible negative impacts to students during the transition.

METHODS The Legacy Curriculum at University of Wisconsin, School of Medicine and Public Health followed the standard medical school educational model, including traditional, departmentbased, clinical clerkships. The ForWard Curriculum began in 2016 and these students entered new integrated clinical experiences in January 2018. Our neurology and psychiatry clinical experiences are integrated with Internal Medicine in Acute Care. Family medicine and ambulatory internal medicine are integrated in Chronic and Preventative Care. Obgyn and pediatric experiences are integrated in Care Across the Life Cycle. Finally, surgery, anesthesia, and other procedural specialties are integrated in Surgical and Procedural Care. We compared NBME scores and passing rates between the final cohort of Legacy M3 students and first cohort of ForWard students for Adult Ambulatory Medicine, Medicine, Neurology, OBGYN, Pediatrics, Psychiatry, and Surgery. Institutional passing thresholds were not changed.

RESULTS NBME scores and passing rates to date for the ForWard Curriculum cohort demonstrate no statistically significant differences from those of the Legacy cohort $(p>0.05)$. Further monitoring of these data is necessary; however, these results indicate no substantive negative effects to student acquisition of 
medical knowledge during the transition period to the integrated curriculum.

CONCLUSIONS Limitations include relatively small sample size due to examining only two cohorts of students. Strengths include examining the same standardized assessment measures.

REFERENCES Curriculum Inventory and Reports (CIR) Initiatives - AAMC. Association of American Medical Colleges, AAMC, aamc.org/initiatives/cir/427196/27.html. Accessed 2018 October 15

\section{ASSESSMENT}

Poster: 105

Voluntary Audience Response System Self-Assessment Quiz Performance Correlates with Exam Performance in Biochemistry and Genetics

Chantal Richardson, Emmanual Segui

${ }^{1}$ Alabama College of Osteopathic Medicine, Dothan, AL 36301 USA

PURPOSE Many medical school use audience response system to engage student in active learning during didactic sessions. Our study examines medical student voluntary use of the audienceresponse system self-assessment quizzes as a checkpoint for comprehension and learning of lecture material presented during a large biochemistry/genetics class. We hypothesize that students will actively participate in the self-assessment quizzes and that there will be a performance correlation between on the selfassessment quizzes and exams.

METHODS First-year osteopathic medical school students $(n=$ 160) were given voluntary audience response self-assessment inclass quizzes during each biochemistry/genetics lecture. The class is broken down into five educational blocks with an average of four to seven biochemistry/genetics lectures in each block. At the end of the block, students were assessed with a culminating exam. Participation and performance scores on the self-assessment quizzes during each educational block was reported via TOP HAT audience response system. The average performance score was plotted against the average biochemistry/genetic exam performance for each of the five educational blocks.

RESULTS The average voluntary participation on the selfassessment quizzes was $81.2 \%$. The average scores on the selfassessment quizzes were statistically similar to the average exam score for each educational block. Using the Pearson correlation, we found that the average audience response self-assessment quiz performance was significantly $(p=0.41)$ correlated with average exam performance $(r=0.89)$.

CONCLUSIONS Audience responses system self-assessment quizzes allow students to actively participate in the biochemistry/genetics didactics. This study shows that students performance on voluntary selfassessment in-class quizzes and on the corresponding exams are closely correlated. With additional studies, we hope to show that the in-class quizzes can be used to accurately predict how students will perform on exams.

\section{ASSESSMENT}

Poster: 106

Do MCQ Quiz Scores Predict Step 1 Scores to the Same Degree If the Quizzes Are No Longer Part of the Course Grade?

Kathryn Moore, Karly Pippitt, Candace Chow, Jorie Colbert-Getz

${ }^{1}$ School of Medicine, University of UtahSalt Lake City, USA
Award Nominee

PURPOSE Scores from multiple-choice question (MCQ) assessments share a moderate-to-strong relationship with Step 1 scores. It is unknown if this relationship varies by the stakes of the assessment (i.e., whether the assessment is graded). If scores from graded assessments predict Step 1 scores, but scores from identical but ungraded assessments do not predict Step 1 scores, this may suggest students are not motivated to perform their best for ungraded/formative assessments. If, however, there is no difference in predictive ability, this would provide evidence that assessments offer valuable learning experiences even if ungraded and formative in nature.

METHODS Participants were University of Utah SOM students matriculating in $2014(N=100)$ and $2016(N=131)$. Students in the graded cohort completed 17 MCQ quizzes across 6 courses in years 1-2 while students in the ungraded cohort completed 19 MCQ quizzes. Both cohorts completed Step 1 at the end of year 2. Quiz performance contributed to $36-50 \%$ of course grades for 2014 matriculates (graded cohort). The testing environment and majority of quiz questions were the same for the 2016 matriculates, but performance did not contribute to course grades (nongraded cohort). Correlations between quiz and Step 1 scores were computed; linear regression was used to determine if quiz scores significantly predicted Step 1 scores.

RESULTS Average quiz scores were $81 \%(\mathrm{SD}=6 \%)$ for the grade cohort and $80 \%(\mathrm{SD}=6 \%)$ for the non-grade cohort. There were strong correlations between quiz and Step 1 scores for the grade cohort $(R=0.79)$ and non-grade cohort $(R=$ $0.63)$. Quiz scores significantly predicted Step 1 scores for the grade cohort, $\mathrm{F}(1,98)=165.81, p<0.001$, and non-grade cohort, $\mathrm{F}(1,129)=84.59, p<0.001$.

CONCLUSION Regardless if used for grading or formative purposes, quiz performance shared a strong relationship and predictive ability with Step 1 performance. This may suggest that assessments need not count towards course grades in order to motivate students.

\section{ASSESSMENT}

Poster: 107

The Use of Reflective Eportfolios as an Assessment Tool of Learner Development from Undergraduate Through to Postgraduate Study

Paula Smith, Uzma Tufail-Hanif

${ }^{1}$ University of Edinburgh, Edinburgh, Scotland

PURPOSE The ePortfolio is being increasingly utilised in medical school curricula and postgraduate training programmes to monitor the development and improvement of learners. Reflective ePortfolios have been used since 2013/14 in a two-year online part-time $\mathrm{ChM}$ in Urology programme at our institution, requiring postgraduate students to learn from professional experiences out with formal teaching. This year, we introduced a similar assessed ePortfolio to the new BMedSci Surgical Sciences course for undergraduate medical students. Here, we evaluate its perceived effectiveness in developing student autonomy and self-reflection skills.

METHODS In 2017/18, students enrolled on the two courses $(n=$ 12 undergraduate and $n=12$ postgraduate) were asked to critically reflect on their experiences, actions and learning under the headings: Quality Improvement and Patient Care; Literature Evaluation Skills; Research and Experimental Design; SelfLearning Abilities and Habits; and, for ChM only, Teaching Skills. Anonymised, interim student feedback on the process has been gathered using online questionnaires. 
RESULTS Eleven students completed the questionnaire (46\% response rate); undergraduate and postgraduate responses were essentially comparable. None of the respondents had any previous experience of keeping a portfolio; 11/11 indicated it is a new learning experience for them. Encouragingly, no-one declared an anxiety about revealing their weaknesses. The majority of respondents find the ePortfolio helps them reflect on their approach to study (82\%) and identifies where they need to improve $(55 \%)$. Two-thirds (7/11) rate the ePortfolio as beneficial and challenging. Whilst $27 \%$ indicated that they found it difficult and confusing, most described it as valuable $(73 \%)$.

CONCLUSION Reflective ePortfolios provide opportunities to give students autonomy, and develop their learning across and between different courses, which they can continue into their postgraduate training and beyond. To be effective, teaching staff need to provide detailed guidance on the assessment requirements, such as exemplars, given that ePortfolios are likely to be a novel endeavour for many students.

\section{ASSESSMENT}

Poster: 108

\section{A Benchmark-Anchored Path for Success on Step 1 and Level 1 Licensure Exams}

Maria Cole ${ }^{1}$, Kerin Fresa ${ }^{2}$, Marcus Bell ${ }^{2}$, Dawn Shell, Linda Adkison ${ }^{1}$ School of Medicine, University of Missouri, Kansas City MO, USA

${ }^{2}$ Philadelphia College of Osteopathic Medicine, Philadelphia PA, USA

${ }^{3}$ School of Medicine, Trinity Medical Sciences University, Ribishi, St. Vincent and the Grenadines, W.I.

\section{Award Nominee}

Abstract

PURPOSE Residency program directors increasingly use a USMLE Step 1 or COMLEX Level 1 score as an important screening point for applicants. Low scores and repeated failures can result in poor outcomes for students. Thus, the development of benchmarks supported by data that identify student progress and provide evidence of preparedness lead to better prepared students with better individual and institutional outcomes. This study is a collaboration between three different medical schools to compare data and develop shared best practices.

METHODS Data were collected for analyses from a US 6-year allopathic medical school, an osteopathic medical school, and a Caribbean allopathic medical school. All schools collect data for students taking USMLE Step 1 and one school collects data for students taking COMLEX Level 1. These data include: course performance, NBME discipline exam results, commercial 8-hour simulation exam results, an internal comprehensive exam, and the NBME Comprehensive Basic Science Exam results. Data for multiple cohorts are combined and association studies were performed to determine Pearson's coefficient and significance.

RESULTS Outcomes demonstrate the following for COMLEX Level 1 exams: Specific course performance can be associated with Level 1 scores COMSAE D, an internal comprehensive exam, and an 8-h COMBANK exam are correlated with Level 1 scores. Outcomes demonstrate the following for USMLE Step 1 exams: NBME basic science exams and a commercial 8-h exam are correlated with Step 1 scores The NBME Comprehensive Basic Science Exam is correlated with Step 1 scores. These results led to institutional-determined benchmarks for each student and students to achieve prior to registering for USMLE Step 1 or COMLEX Level 1. The benchmarks led to improved students performances for first time pass rate and mean score.

CONCLUSION These studies demonstrate the power of shared information and collaboration between schools and support schools efforts for students' success.

\section{ASSESSMENT}

Poster: 109

Preliminary Analysis of the New MCAT, Student Progression, and USMLE Step 1 Performance

\section{Linda R Adkison}

${ }^{1}$ School of Medicine, Trinity Medical Sciences University, Ribishi, St. Vincent and the Grenadines, W.I.

PURPOSE In 2015, a new Medical College Aptitude Test was introduced in the USA. Many schools include more non-cognitive measures of applicants, but the MCAT remains a prominent tool. This study assesses student progression and Step 1 performance with new MCAT scores.

METHODS Data from students matriculating in 2016 were collected: matriculation date, undergraduate grade point average, new MCAT score, clinical clerkship entry date, and first attempt Step 1 performance. Forty-four of 106 students met the inclusion criteria for the study. MCAT scores were sorted into the upper, middle, and lower range and compared with data presented at the AAMC Annual Meeting in November 2018. Start dates for each of these students was then reviewed for each of score range.

RESULTS Data show that the majority of Trinity students who matriculate had MCAT scores in the Middle (495-504) and Lower third of the score range (472-495). US schools admit a majority of students from the Upper third (505-528) while Trinity matriculated about $10 \%$ of students in this group. Among the 2016 cohorts, $55.8 \%$ of students had MCAT scores in the Lower third of scores. There is greater attrition and delay among this cohort in starting clerkships but students completing Step I in these three cohorts have a $100 \%$ pass rate; AAMC data demonstrate $80-98 \%$ pass rate. Data show that $100 \%$ of students who take Step 1 in the Top cohort start clerkships on-time. Ninety percent and $67 \%$ of students with Middle and Lower scores start on-time, respectively. AAMC reports that in larger US cohorts, 95\%, 93\%, and $70 \%$ of students start clerkships on-time each cohort, respectively. CONCLUSION Data show that students with low MCATs and acceptable grade point averages can be successful in a medical curriculum and pass Step 1 on the first attempt.

\section{ASSESSMENT}

Poster: 110

Leveraging Student Perception of Assessment Performance to Support Learning

Brock Mutcheson, Andrew Binks, Renee Leclair, Emily Holt

${ }^{1}$ VTCSOM, Roanoke, USA

\section{Award Nominee}

PURPOSE The overall goal of the study was to determine and refine the efficacy of student-support services intended to improve student performance. For this particular analysis, the research team investigated and described the extent to which student perceptions of their own assessment performance were linked to data aberrations detected using traditional data forensics.

METHODS The study so far has included 102 first year medical student observations and seven assessments; three formative and four summative exams. In total, there have been 1090 item-level observations. During exam review sessions, participating first year medical students indicated the type of error they believed they had made 
on a questionnaire developed through a literature review conducted by the research team. The types of errors were categorized into two major groups; Type A, a test-taking error (e.g. poor question interpretation, misreading a question) or Type B, a lack of content mastery (i.e. they didn't know the answer). Student perceptions were investigated by student demographic and academic performance characteristics and various item-level characteristics. For this analysis, the team focused on data aberrations detected on individual items an examinee was anticipated to answer correctly based on response patterns, but that the examinees actually answered incorrectly (Meijer 1994). The estimated error rates were then associated with the preliminary summary findings.

RESULTS Several data aberrations were identified and the error rate was found to be significantly correlated with multiple type-A error categories (i.e. poor test-taking) identified by students.

CONCLUSIONS This analysis provided validity evidence for one intended use of the questionnaire and promising evidence for students' abilities to recognize and explain assessment errors. Moreover, this analysis demonstrated the value of including an alternative post hoc measure to the assessment feedback process with the ultimate goal of improving learning support.

\section{ASSESSMENT}

Poster: 111

Didactic-Year Oral Examination Grades as Predictors of Performance on Future Written Licensure Examinations

Ellen D. Feld, Patrick C. Auth, Charles Stream, Daniela ChelesLivingston

\section{${ }^{1}$ Drexel University, Philadelphia, USA}

PURPOSE Oral examinations have been used for many decades in medical education. The validity and reliability of traditional oral examinations (where student and examiner engage in a relatively free-form discussion) have been questioned. Structured oral examinations, where the examiner asks the student a predetermined set of questions, have been found to be more reliable. Our program uses structured oral examinations; where each student discusses one out of a predetermined list of diseases, and faculty grade using a checklist; in our didactic-year Clinical Medicine I and II courses. We examined the relationship between oral examination grades and subsequent scores on the Physician Assistant National Certifying Examination (PANCE), which students take after graduation.

METHODS We performed an analysis of the examination grades and scores of 352 students in the graduating classes of 2013-2017, using Clinical Medicine I and II oral examination grades and PANCE scores. RESULTS Both Clinical Medicine I and Clinical Medicine II oral examination grades have a small but significant correlation with PANCE scores.

CONCLUSION Clinical Medicine I and II oral examination grades are weak predictors of PANCE performance. There are several possible reasons for the lack of a strong correlation: each student receives a randomly selected oral examination topic, therefore not all students receive the same examination; any individual student might score differently if given a different topic. The PANCE, on the other hand, is a comprehensive examination, covering all body systems and medical tasks. An alternative explanation for the weak correlation is that oral examinations may evaluate different skills than written tests, such as the ability to perform in a social setting and to think-on-ones-feet.

\section{ASSESSMENT}

Poster: 112

Retention of Medical Knowledge Across the First Year of Medical School
Carrie Bailes, Mary Caldwell, Renee Chosed, Anna Blenda, Matthew Tucker

${ }^{1}$ School of Medicine Greenville, University of South Carolina, Columbia, USA

\section{Award Nominee}

PURPOSE Medical students are tasked with absorbing a vast amount of medical knowledge. Because of this, it is important to assess how much of that knowledge they retain as well as the depth of memory for that information. Here, we retested students on a subset of questions from their Molecular and Cellular Foundations of Medicine summative exam 10.5 months after they first sat for the exam. In addition to re-assessing student performance using the same multiple choice format (cued recall), we also tested how well students could answer the questions without seeing the answer choices (free recall).

Methods Second-year medical students ( $N=46,25$ females) reported to the same location of the original exam and used the same testing software. Fifty of 104 questions were selected from the original exam based on whether the item could be answered from memory without seeing the answer choices. The question stem was displayed with a text box below it to allow for the free recall of an answer. After an answer was entered, the original answer choices were shown, and students selected the best answer.

Results The students' average on the original exam was $87.2 \pm$ 5.5\%. 10.5 months later, students answered correctly $53.9 \pm 9.6 \%$ of the items, which was $62 \%$ of their original scores. Free recall rates (i.e., recollection without seeing the answer choices) were considerably lower, with students correctly answered $15.8 \pm 9.2 \%$ of the questions. Initial exam performance did not correlate with retest scores.

Conclusions Understanding how much information is retained in medical school is important for evaluating teaching effectiveness and the difficulty of course content. Understanding differences in free vs. cued recall may provide insights about how deeply information is memorized. Testing protocols can be developed to compare retention rates between modules and across different time spans.

\section{ASSESSMENT}

Poster: 113

Learner Assessment 2.0: Embedding Remediation into the Assessment Strategy

Leah Sheridan ${ }^{1}$, Andrea Barresi ${ }^{1}$, Paige Gutheil ${ }^{1}$, Sheila Chelimo ${ }^{2}$

${ }^{1}$ Heritage College of Osteopathic Medicine, Ohio University, Athens, USA ${ }^{2}$ Ohio University, Athens, USA

Purpose Assessment is a systematic process that involves designing, collecting, interpreting and using information to ascertain student performance in a learning environment with the primary goal of improving students learning and development. Assessment is not separate from the learning process but a crucial part of medical education, and a robust assessment program should strategically and seamlessly embed learner remediation.

Methods The Ohio University Heritage College of Osteopathic Medicine launched its novel Pathways to Health and Wellness Curriculum in Fall 2018, which emphasizes integration of foundational science and generalist clinical concepts, application to patient care, assessment for learning, and personalization of the educational experience. The first of four semester-long courses, Wellness, 
employs integrated assessments that learners use to hone their Osteopathic core competencies for practice over time. Each assessment is designed for learning and creates opportunities for learner monitoring of progression toward specific learning outcomes. This formative assessment strategy encompasses a series of low-stakes quizzes and exams designed to assess learner mastery of new content as well as learner retention of past content from the previous quiz or exam.

Results For each assessment, faculty evaluated the extent to which students mastered both the new and preceding content to identify learner areas of mastery and for remediation. Each subsequent assessment is then informed by learner performance, personalized to prospective areas for improvement, while concurrently assessing new knowledge and skill.

Conclusion With future cohorts, the Wellness Course aims to personalize exams at the level of the individual learner by offering each learner the opportunity to demonstrate improvement upon past performance. We believe that the merging of assessment and remediation strategies fosters the culture of life-long learning and improvement expected of medical professionals today.

\section{ASSESSMENT}

Poster: 114

Causes of Variation in the Predictive Validity of Formative Assessments in an Organ-System Based Preclinic Curriculum

Jason Booza, Paul Walker, Matt Jackson

${ }^{1}$ School of Medicine, Wayne State University, Detroit, USA

\section{Award Nominee}

Purpose In 2018, the Wayne State University School of Medicine launched a new preclinical organ-system curriculum with facultyauthored weekly formative assessments coupled to end-of-unit summative exams prepared through the National Board of Medical Examiners customized assessment system. Preliminary evaluation indicates that while the formative assessments were moderately-tohighly predictive of summative performance, there was variation in predictive validity of low and high performers. Formative assessment tended to overpredict for low summative performers and underpredict for high summative performers. While medical education literature is replete with assessment best practices, there is a paucity of studies addressing the variation of learners performance on formatives assessments. Our purpose is to understand causes of variation affecting student learning and assessment, as well as to address this gap in the literature.

Methods A mixed-method approach analyzed formative assessment practices routinely captured through ExamSoft. Data was collected from weekly formative assessments completed on or off campus during an open period from Friday afternoon to Sunday evening. Randomization was achieved with a stratified random sample survey of medical students across 3 levels of summative performance.

Results We found high summative performing students took the formative assessments earlier and spent less time preparing. They were also more likely to use the post-exam review and performance summary features of the formative assessments in preparation for the summative exams. These students viewed the system as being a valuable part of their learning strategy. Low and mid summative performers completed formative assessments late and many reported that they did not utilize the postexam review and performance summary features.

Conclusion The predictive validity of formative assessments appear be affected by the strategy of preparation, use of the performance summary features, and self-fulfilling views of the formative assessment process. These findings may shape targeted interventions to improve student learning strategies.

\section{ASSESSMENT}

Poster: 115

Medical Student Optimism: Hopefulness and Confidence About the Future as Impacted by Personality and Gender

Diane Brown, Jeff Fritz, Koenraad De Roo, Amy Prunuske, William J. Hueston, Kristina Kaljo, Craig Hanke, Molly Falk-Steinmetz ${ }^{1}$

${ }^{1}$ Medical College of Wisconsin, Milwaukee, USA

Purpose Optimism is the hopefulness and confidence about ones future. It is an important facet of emotional intelligence and wellbeing that helps medical students meet academic challenges and think positively towards graduation, residency, and independent practice. As an enduring intrinsic trait, it is linked to personal disposition and therefore impacted by their personality. The purpose of this study is to analyze the relationship of medical student optimism and personality as moderated by gender.

Methods In 2017/18, 205 of 500 M-1/M-2 medical students (106 males/99 females) voluntarily completed these self-reported surveys: (A) Trait Emotional Intelligence Questionnaire (TEIQue-sf) to measure optimism (scale: 1 = completely disagree $/ 7$ = completely agree), and (B) Five Factor Personality Inventory (scale:1 = very uncertain $/ 5=$ very certain). Inter-item reliability determined with Cronbachs alpha. Differences in mean scores analyzed with independent $t$ tests and Cohens $\mathrm{d}$ effect sizes. Pearson correlations (r) and stepwise multivariate linear regressions used for predicting optimism scores from personality. IBM ${ }^{\circledR}$ SPSS ${ }^{\circledR} 24.0$ generated statistical analysis. This research approved by institutions IRB.

Results Optimism (alpha $=0.6)$ mean scores were significantly higher $(d$ $=0.3, p<.033)$ for female students $(\operatorname{mean}(\mathrm{sd})=5.8(1.0))$ than male students $(5.4(1.3)$ ). Eighty-four percent of optimism scores were above the instruments midline score $=4.0$. Optimism was significantly $(p<$ $.050)$ correlated to neuroticism $(r=-0.5)$, agreeableness $(r=0.3)$, extroversion $(r=0.3)$, and conscientiousness $(r=0.2)$. Linear regression results for female optimism $(\mathrm{R}=0.38, p<.001)$ were predicted by neuroticism (beta $=-0.5$ ), extroversion $(0.2)$, and openness $(0.2)$. Linear regression results for male optimism $(\mathrm{R}=0.51, p<.001)$ were predicted by neuroticism (beta $=-0.3$ ), extroversion $(0.2)$, and agreeableness $(0.2)$.

Conclusions Optimism scores were positive for most medical students, but significantly higher for female students than male students suggesting greater hopefulness and confidence in their future. Emotional personality traits such as neuroticism and extroversion directly impacted optimism for all students. However, different cognitive personality traits such as openness to experiences assisted female student's optimism, whereas being agreeable helped male students.

\section{CURRICULUM}

Poster: 200

\section{The Rotating Radiologist}

Peter Nesbitt, Molly Johannessen

${ }^{1}$ Lake Erie College of Osteopathic Medicine, Erie, USA

Purpose A physician equipped with basic radiological information and reading skills will provide superior care for all patients. Unfortunately, very few physicians receive proper instruction needed during their medical school career and the foundation of radiological knowledge needs to be strengthened. Many schools have acknowledged this problem and have attempted to address it, 
and there is a call for a well-developed and uniform curriculum to train radiology to all medical students. In addition to medical schools, survey data of physicians show support and understand the necessity for a curriculum that will bring better education to future physicians.

Methods This project presents the development of an online radiology curriculum for use by any medical schools or medical students intending to expand their radiological knowledge. To efficiently integrate the material into students learning, a few approaches may be used: A clinical radiology four-week clerkship covering all avenues of radiological imaging and interpretation or a two-week supplementary educational curriculum. This flexible curriculum allows learning to fit in many different shapes and forms of the already full curricula of medical school. Furthermore, allowing this material online will allow learners to access it at any time from any location when they are in need of refreshing knowledge.

Results and Conclusion The goal is to educate future physicians, while also alleviating the overwhelming prospect of analyzing images by developing a widely accepted systematic approach to image analysis. The reality is that majority of radiological reports come back normal. With a focus on clinical medical students, this learning tool will enable students to view a radiograph in a reallife context and know exactly where to start. An online resource featuring a partnership of instruction and practice radiological reports will help the medical community to become better prepared with radiologic knowledge.

\section{CURRICULUM}

Poster: 201

Do Medical Students Appreciate Lifestyle Medicine and Are They Confident In Their Skills? A Needs Analysis for Educational Interventions

\author{
Magdalena Pasarica, Denise Kay \\ ${ }^{1}$ College of Medicine, University of Central Florida, Orlando, USA
}

Purpose Two decades ago, integrating nutrition into the preclinical education of medical professionals received a strong push assuming that it will translate clinical skills and ultimately to clinical practice. As strong data on the benefit of changing other behaviors (like exercise and sleep) became available, the assumption was that it will also be integrated. Currently, studies show that medical providers are not using lifestyle changes for managing and/or preventing chronic diseases (defined as lifestyle medicine-LM), which was interpreted by American Medical Association (AMA) as a gap in LM training. Our purpose was to determine what items need addressing in the clinical years for filling the gap in LM education.

Methods Medical students were surveyed during the first clinical year using a 5-point Likert scale about perception related to LM (from strongly disagree-1 to strongly agree-5) and about confidence in their LM skills (from not at all confident-1 to extremely confident-5).

Results Students agreed that lifestyle management recommendations are a valuable use of limited patient-doctor time (mean $4.4, \mathrm{SD}=0.7)$; a valuable tool for prevention and management of chronic diseases (mean 4.9, $\mathrm{SD}=0.3$ ); and worth their time (mean 4.7, $\mathrm{SD}=0.5$ ). Student reported self-confidence in prescribing personalized lifestyle advice to patients with multiple common conditions (mean 3.2, SD $=0.8$ ), obtaining comprehensive lifestyle history (mean $3.8, \mathrm{SD}=0.8$ ); assisting patients with setting personalized, clear lifestyle goals (mean 3.9, $\mathrm{SD}=0.1$ ); and using motivational interviewing with patients for improving the outcomes of lifestyle interventions (mean 3.2, $\mathrm{SD}=0.8$ ).

Conclusions Our study showed that both assumption were incorrect. LM requires an advanced clinical skill that needs formal expansion into the clinical years for learners to develop the confidence needed to integrate LM into their practice. When addressing the LM gap, educators should allow more time and effort for targeting the lower scored items, especially gaining confidence in providing efficient lifestyle management.

\section{CURRICULUM}

Poster: 202 The Effects of Early Clinical Exposure on the Professional
Assessment Rating Scale

Lauren M. Granat, Anna Weinstein, Donna McMahon, Christine Hutak, Bhuma Krishnamachari, William Blazey, Thomas Chan

${ }^{1}$ College of Osteopathic Medicine, NYIT, New York, USA

Purpose Many medical schools in the USA are transitioning to curricula that include clinical experiences during the didactic years. There are numerous advantages to early clinical exposure: improved physical exam performance, increased confidence in treating patients, and better integration of clinical and basic sciences. New York Institute of Technology College of Osteopathic Medicine (NYITCOM) has two distinct curricular tracks: the Lecture-Discussion Based (LDB) track, which primarily utilizes traditional lectures and the Doctor-Patient Continuum (DPC) track, which is problem-based. About $88 \%$ of the student body is enrolled in the LDB curriculum. Due to the small size of the DPC cohort, students have more opportunities for early clinical exposure, including shadowing and simulation-based patient and mannequin encounters. Students at NYITCOM are assessed using the Professional Assessment Rating Scale (PARS). The PARS is comprised of the following components: Rapport, Empathy, Confidence, Nonverbal Communication, Communication, Listening, Counseling, and Physical Examination. Students are given scores ranging from 1-9, with those achieving a score less than 4 flagged for intervention.

Methods PARS scores were compared between the curricular tracks using $t$ tests, with $p=0.05$.

Results One thousand eighty-six LDB students and 129 DPC students were included in this analysis. DPC students received higher scores on each aspect of the PARS score, as compared to LDB students ( $p$ 0.0001), though both averages showed satisfactory performance. The aggregate PARS scores for both groups also showed satisfactory performance, with a statistically significant higher average among DPC students vs. LDB students (6.53 (SD 0.44) vs. 6.23 (SD 0.50); $p$ 0.0001)).

Conclusion Additional clinical exposure may be helpful for students, leading to better patient communication and clinical skills. These initial results need be confirmed with a larger study and should account for selection bias using appropriate comparison groups.

\section{CURRICULUM}

Poster: 203

Early Human Development: Integrated Course of Embryology and Genetics

\section{Iuliia Zhuravlova}

${ }^{1}$ Trinity School of Medicine, Ribishi, St. Vincent and the Grenadines

Introduction The integration of the disciplines is one of the main trends, which in many cases remains a challenge 1 . The challenge 
includes not only the organisation of the process itself, but also the level of detail required for each of the integrated courses, as well as the relevance of the taught material. It is widely discussed in the literature, that integrated programs promote deep learning as well as the better retention of knowledge 2, 3 .

Methods The new Early Human Development course included Genetics topics combined with the Embryology. To compare the outcomes, the Embryology standardised questions were used. We have compared the level of students' performance in Embryology part of the material before the course was integrated with Genetics versus after. The students were also surveyed on their perception of the effectiveness of the integrated course.

Results It was identified that the quality of responses to the standardised Embryology exam questions, after it was integrated with the Genetics course increased up to $17.7 \%$ in 2016, and up to $18.3 \%$ in 2017 . In 2018 the tendency remained positive with $18.1 \%$ increased level of students' performance compared to the results before integration. The difference was statistically significant $(p<0.01)$. Overall amount of students evaluated during the period from Summer 2016 semester to Summer 2018 semester was 268. The students' responses regarding the effectiveness of the integrated course were overall positive. Several responses suggested the faculty to rearrange several topics within the course for a better flow of the material. The level of feedback was $60.8 \%$ (163 students).

Conclusion With our study, we have confirmed that integration of the Embryology and Genetics courses might help students to learn Embryology with the better outcomes. We plan to compare the standardised Genetics questions as the next step of our study.

\section{CURRICULUM}

Poster: 204

\section{Integrating Scientific Research into Medical Education Through a} Comprehensive Elective Course

Marek Svoboda, Yasmin Kamal, Roshini Pinto-Powell

${ }^{1}$ Geisel School of Medicine at Dartmouth, Hanover, USA

Award Nominee

Purpose One of the goals of evidence-based medical education is to familiarize future physicians with the scientific method in order for them to comprehend scholarly literature and communicate it to their patients. However, many medical students lack the skills necessary to participate in research themselves. Here we describe an implementation of a comprehensive elective course which is designed to equip interested students with these skills through a series of workshops, one-on-one mentorship, and pursuit of a summer research experience.

Methods The elective course has been implemented for one year. Participating students are selected among first-year medical students based on their motivation to conduct basic, translational, or clinical research. No prior experience is required. Throughout the year-long course, students attend a series of 10 one-hour workshops designed to teach them the skills necessary to engage in research. Topics covered by invited faculty experts include resources management, clinical trial design, scientific writing, and funding opportunities available to medical students. Additionally, each student is paired with a peer mentor to help them navigate the research community. At the conclusion of the course, students complete and present a Specific Aims page based on their proposed study.

Results Nine students participated in the first year of the elective (2017-2018). All students secured a research position for the summer following their first year of medical school and four students also obtained nationally competitive funding. Seventyeight percent of the students indicated that participation in the elective significantly helped them find and secure these positions and $88 \%$ learned valuable research skills not taught in their medical curriculum.

Conclusions M1 participation in a well-designed research elective has the potential to provide immediate value in the acquisition of a summer research position and to stimulate a lifelong interest in scientific inquiry.

\section{CURRICULUM}

Poster: 205

Integrating a Longitudinal Systems and Safety Science Thread into a Four-Year Medical School Curriculum

Sarah H. Parker, Richard C. Vari, Cynda A. Johnson

${ }^{1}$ School of Medicine, Virginia Tech Carilion, Roanoke, USA

Purpose Systems safety is rapidly becoming common parlance in medical schools and hospitals. However, there is limited information available on when and how to integrate this content into medical school curricula. As students move from classroom to clerkship and into residency, it is critical that they know the language of health systems science, and have the capacity to understand system factors that influence team performance. Methods A team of clinicians, medical educators and systems safety experts developed a systems safety thread through all 4 years of the medical school curriculum. After completing a keyword curriculum mapping exercise, we focused on two overall objectives for the new systems safety curricular thread: (1) understanding the language of safety and being fluent in its use, and (2) understanding the science of safety through exploration of human error and individual and team performance limitations.

Results Integration of our new systems safety thread through all 4 years of the curriculum began in fall of 2017. During lectures in their first year, students were taught systems safety using the SEIPS (Systems Engineering Initiative for Patient Safety) model as a framework for systems safety. Throughout the first 2 years, systems safety concepts were addressed via new learning objectives within problem based learning (PBL) cases. Specific learning objectives related to team performance, leadership and systems safety were also integrated within the Interprofessionalism learning domain.

Conclusion Using PBL cases to address this content is a strength and provides students an opportunity to see how systems safety concepts are applied in a clinical setting. Significant development work is currently underway to integrate specific systems safety learning objectives across clerkships and sub-internship rotations including dovetailing our work with similar requirements for graduate medical education. Integrating this content across the curriculum requires significant faculty development to ensure fluency in these concepts.

\section{CURRICULUM}

Poster: 206

Nutrition in Medicine: Educating Medical Students on the PlantBased Diet as a Way to Combat Preventable Diseases

Kristina J. Van Nuys, Robyn Dreibelbis

${ }^{1}$ Western University of Health Sciences, Pomona, USA

Purpose 
Fewer than $14 \%$ of physicians believe they are properly trained in nutrition counseling, yet research shows that the chronic disease risk of chronic diseases can be decreased through healthy nutrition. Nutrition in Medicine (NiM) is an evidence-based, 8-session elective lecture series centered on nutrition and human health. We wanted to understand the impact that nutrition education has on medical students personal dietary habits as well as their confidence, willingness, and motivation to counsel others about nutritions impact on health.

Methods First- and second-year medical students completed a survey before and after the 2017/2018 NiM lecture series. Surveys included questions about personal dietary habits, willingness and motivation to change personal dietary habits, confidence and motivation to counseling others on plant-based diets, and confidence in nutritional knowledge. Chi-square tables were used to test for statistical significance with $95 \%$ confidence intervals.

Results Four hundred seventy-two students completed the surveys; 264 students had never attended a lecture (Group 1) and 208 attended at least one lecture (Group 2). Statistical significance was found in all categories of the study comparing post-surveys between Group 1 and Group 2. Students who attended at least one lecture were found to adopt a more plant-based diet, were more willing and motivated to adopt a plant-based diet, and were more willing, confident, and motivated to counsel others on nutritions impact on health and on the plant-based diet.

Conclusion Teaching medical students about nutrition is a unique way to solve the national burden of chronic disease. Nutrition education changes medical students diets, as well as their confidence and motivation to counsel others in nutrition as medicine. Mandating nutrition as a core competency of medical training has the potential to change the health of our nation.

\section{CURRICULUM}

Poster: 207

SAFE—a Scaffold Curriculum to Teach Safe, Appropriate, Timely and Value Based Imaging to Medical Students

Janet A. Neutze, Allene S. Burdette, James M. Brian, Pamela L. Brian, Alison L. Chetlen

${ }^{1}$ Penn State College of Medicine, Hershey, USA

Purpose Students with diverse specialty interests often take a diagnostic radiology elective. Classically, radiology electives teach radiologic anatomy and pathology, reviewing the basics needed for clinical practice. Now at the forefront of radiology education, the issue of cost and safety are increasingly important to the public. Teaching responsible utilization of imaging and its relative costs can be incorporated into radiology education. A new curriculum was created to align with the American College of Radiology (ACR) Imaging 3.0 goals of providing appropriate, timely and value-based imaging to all patients and clinicians. Students will gain skills to be able to choose the safe, appropriate imaging.

Methods We created an educational program to improve patient care and increase value utilizing a new scaffold curriculum for medical students and learners of all levels. Medical Student Education directors refined diagnostic radiology elective goals to not only fulfill students expectations but also to provide them with new non-interpretative skills. This new program includes evidence-based decision making and incorporates health care policy, economics, and patient centered care. The SAFE curriculum includes: Safety -Appropriateness-(interpreting) Films- Expedite and Execute. Without safety and appropriateness practiced first, even the best imaging interpretation may not result in the indicated and desired value-based, patient-centered care.

Results Due to the positive responses from post-program surveys, we plan to broaden applications of the SAFE radiology concept throughout all four years of medical school curriculum in courses such as anatomy, clinical clerkships and other electives. We are also considering the creation of an accomplishment designation to recognize levels of achievement among faculty radiologists (Master Teachers of the SAFE concepts) with plans to integrate junior and senior radiology residents into the teaching curriculum.

Conclusions SAFE radiology is an easily learned, effective method to introduce learners to concepts of appropriate, timely and value-based imaging.

\section{CURRICULUM}

Poster: 208

Assessment of Medical Students' Attitudes and Use of Complementary and Alternative Medicine at a Caribbean Medical School

Benjamin Karabasz

${ }^{1}$ St. George's School of Medicine, St. George's, Grenada

Purpose The goal of this survey was to examine the attitudes and use of Complementary and Alternative Medicine (CAM) of medical students from St. Georges University School of Medicine; the largest international provider of physicians practicing in the USA.

Methods A questionnaire was emailed to 2nd year medical students to collect data regarding respondents knowledge about and attitudes toward $\mathrm{CAM}$ as well as their personal use and experience with CAM modalities. A previously validated survey, The CAM Health Belief Questionnaire was supplemented with questions on student personal use of CAM, future use of CAM modalities to treat patients, and the sources for their knowledge about CAM.

Results The results of this survey indicate current medical students have more personal use of CAM modalities and are more likely to recommend patients and friends for CAM treatment than medical students in past surveys. Specifically, $95 \%$ of medical students have personally found CAM modalities useful, $82 \%$ would recommend CAM to patients, and $75 \%$ would refer patients to a CAM practitioner. Students reported a lack of evidence based knowledge about CAM and a desire for increased CAM education in their medical school curriculum.

Conclusion The experience with and use of CAM modalities for themselves and for future patients has increased compared to previous medical student assessments. This is despite student frustration with the amount of objective and critically reviewed CAM instruction presented in class. Increased student use mirrors trends reported for the general U.S. population and supports the need for CAM education presented in an evidence-based manner.

\section{CURRICULUM}

Poster: 209

The Impact of Student Engagement During Individualized Coaching on Step 1 Performance

Yenya $\mathrm{Hu}$, Andrea Vallevand

${ }^{1}$ Wake Forest School of Medicine, Winston-Salem, USA

Purpose Performance on the United States Medical Licensing Examination (USMLE) Step 1 is one of the major factors that 
can influence residency program selection. To maximize the potential for each student to deliver their best performance, we have developed and implemented a longitudinal, phase-specific, outcome-driven, and individualized Step 1 coaching program throughout the preclinical curriculum. The level of student engagement was monitored during the coaching sessions. We hypothesized that students who are engaged in the coaching process benefit more, as they are more willing to evaluate their strategies and monitor progresses, and thus perform better on Step 1.

Methods A regression equation, based on preclinical performances, was generated to calculate the predicted Step 1 scores. Group and individualized coaching sessions were conducted throughout the preclinical phase and more intensified in second year. Analysis of variance (ANOVA) was undertaken to establish whether there are differences between the engaged and not-engaged groups on predicted and actual scores, and the difference between two.

Results Multiple $\mathrm{R}$ for the regression equation equaled $0.839(p<.001)$ and variance accounted equaled $70.4 \%$. ANOVA revealed no difference in predicted Step 1 scores between the engaged $(n=75)$ and not-engaged $(n=23)$ groups $(\mathrm{F}[1,96]=1.864, p 0.175)$. The engaged group performed significantly better on actual Step 1 scores $(F[1,96]=14.546, p$ $.001)$ and on the difference between predicted and actual Step 1 scores $(F[1,96]=19.007, p .001)$. Nineteen students did not participate in coaching. No statistically significant differences between their predicted vs. actual Step 1 scores were found $(\mathrm{F}[1,37]=0.051, p .823)$.

Conclusion Being engaged in the coaching sessions promotes accountability, self-reflection and deeper outcome-driven learning, which may contribute to a better performance on Step 1 .

\section{CURRICULUM}

Poster: 210

Lessons in Medical Ethics: a Novel Peer-Facilitated Medical Ethics Curriculum

Mikalyn DeFoor ${ }^{1}$, Lauren East ${ }^{1}$, Shawna McCafferty ${ }^{2}$, Paul Mann ${ }^{1}$, Carol Nichols ${ }^{1}$

${ }^{1}$ Medical College of Georgia, Augusta University, Augusta, GA 30912 USA

${ }^{2}$ Medical University of South Carolina, Charleston, SC 29425 USA

Purpose Due to the shifting trends of medical education away from traditional lectures, peer-facilitated, case-based instruction for teaching medical ethics is an innovative approach. The introduction of ethical principles and opportunities to practice them during the pre-clinical curriculum is essential to the development of future physicians.

Methods Quarterly, peer-facilitated medical ethics case discussion sessions were added to the Year 1 curriculum. Each session was led by a group of second year medical students and incorporated a case with an ethical dilemma, a relevant review article, discussion questions and a short pre-and post-session survey. Students were asked for feedback on the post-session surveys and on an end of year curriculum survey.

Results For the 2017-18 academic year, post-session survey results indicated better understanding of ethical dilemmas, including consideration of social disparities in biospecimens, patient privacy, reproductive assistance and informed consent. In response to the end of the year survey $(\mathrm{n}=$ 82 ), $74.5 \%$ of students were neutral, agreed, or strongly agreed that the peer-facilitated discussions contributed to their development as a future physician. Narrative responses about the sessions were also encouraging with students requesting more time for discussion.

Conclusions Peer-facilitated medical ethics case discussions appear to be a novel and effective strategy for teaching medical ethics to students in the pre-clinical curriculum. The format allows students to discuss ethical principles and dilemmas in a nonthreatening, peer-facilitated environment. This model for teaching medical ethics is both effective and easy to implement in other medical education settings and programs.

\section{CURRICULUM}

Poster: 211

Translational Neuroscience Research Mentorship. A Platform to Integrate Scientific Research into Medical Education

\author{
Alberto Musto, Andrew Ojeda \\ ${ }^{1}$ Eastern Virginia Medical School, Norfolk, USA
}

Abstract Scientific training in medical education could contribute to improve the understanding of clinical problems and improving health outcomes. This mentorship experience focuses on translational neuroscience research concepts in the medical curricula that will contribute to understanding the nervous system, pathologies associated, differential diagnosis, scientific method, laboratory techniques, biomarkers, and novel therapeutic targets.

Methods This mentorship, promoted for first and second year medical students, was developed during the current curricular year. It used the current content of medical neuroscience with knowledge of basic and translational research to study real cases from clinical trials and current scientific research publications. Students selected a problem, create a hypothesis, and develop an approach to test in the laboratory. Assessments were based on (A) successful identification of scientific premises, description of the experimental designs, summary of results and alternative methods, impact of disease, presentation of results in of poster or manuscript; (B) laboratory techniques, presentation and writing skills, professionalism, and teamwork; (C) feedback was conducted before and after the program to evaluate this mentorship as a learning experience.

Results During 2017-2018, 25 students, which represent a total of $14 \%$ of the medical students population requested to participate in this mentorship. They contributed to four publications in top-tier journals (one invited for resubmission), six abstract and poster presentations to professionals meetings, in addition to strengthening their medical scholarly in preparation for residency.

Conclusion This mentorship will allow the continuing improvement of the students approach to current scientific problems and medical necessities by promoting critical thinking, creativity, building strategic teamwork, and imparting the students with confidence to continue research in medical neuroscience.

\section{CURRICULUM}

Poster: 212

\section{An Innovative Method to Promote Quality in Peer Feedback}

Ashleigh CM. Bull, Alexander Beckstead, Shreya Sreekantaswamy, Rachel Huynh, Rachel Tsolinas, Cecilia Peterson, Veronica M. Urbik, William West, Janet Lindsley

${ }^{1}$ School of Medicine, University of Utah, Salt Lake City, USA

Purpose Peer feedback, though common in undergraduate medical education, lacks a consistent system to ensure quality. The University of Utah School of Medicine (UUSOM) integrated a quality grading system into the peer feedback component of Team Based Learning (TBL). Current TBL feedback 
methodologies focus on self-assessment and peer feedback of a student's performance. There is no established modality, however, in which students grade the quality of the feedback they receive. Methods In preclinical coursework, UUSOM students provide feedback to their TBL teammates and rate the feedback they receive utilizing a 5point rubric: Was this feedback actionable? Was this feedback specific? Was this feedback respectful? Was at least one positive behavior identified? Was at least one constructive suggestion given? The peer ratings students receive for their feedback count toward their final grade. The main limitation of this rubric is the potential for social pressure resulting in grade inflation. Student TBL feedback and ratings for MS2021 (5 courses) and MS2022 (2 courses), along with MS2020 peer feedback (prior to implementation of the 5-point rubric) are utilized. First, the rubrics impact on feedback quality will be assessed by comparing blinded researcher ratings (using the 5 point rubric) for the MS2020 and MS2021/ MS2022 cohorts. Next, the accuracy of the student feedback rating within the MS2021 and MS2022 classes will be assessed by comparing blinded ratings to peer ratings. Finally, longitudinal improvement in feedback quality will be evaluated by comparing blinded MS2021 feedback ratings over five courses.

Results Preliminary analysis suggests a measurable improvement in feedback quality with the implementation of the 5-point rubric.

Conclusions This method provides a quantitative tool useful for measuring and improving the quality of student feedback. This 5-point rubric can be easily implemented into an established curriculum to improve the quality of peer feedback.

\section{CURRICULUM}

Poster: 213

\section{Multiinstitutional Faculty Perspectives on Transitioning to Active Learning}

\author{
Laurel Gorman ${ }^{1}$, Monica Baily ${ }^{2}$, Andrea Berry ${ }^{2}$, Magdalena Pasarica ${ }^{1}$ \\ ${ }^{1}$ College of Medicine Dept Medical Education, UCF, Orlando, USA \\ ${ }^{2}$ College of Medicine Dept Faculty Life \& Development, UCF, Orlando, \\ USA
}

Purpose While most medical education programs dictate that faculty employ active learning methods (ALMs) and reduce didactics, limited data exists on faculty identity and perspectives as curricula transition, shifting traditional teaching roles. To gain insight into faculty perspectives, we conducted a focus group of medical educators during the 2017 IAMSE meeting utilizing audience responses systems (ARS) and group discussion. The purpose of this poster is to share themes arising from this session to inform a larger audience about faculty perspectives in this age of curricular transition.

Methods Fifty-five medical educators with diverse backgrounds and levels of experience anonymously answered ARS questions and participated in small groups $(N=6-8$ per group, 8 small groups) followed by large group sharing using gallery walk deliverables. ARS and small group topics included self-identity, ALMs motivation, and barriers limiting success. Themes were determined after the activity by reviewing anonymous group products and ARS survey data.

Results Survey data suggested faculty perceived ALMs were most effective for promoting retention ( $89 \%$ mostly/strongly agreed) but less agreed that ALMs received positive evaluations (25\%) or were time efficient (42\%). The greatest motivator to using ALMs was increasing engagement ( $22 \%$ selected as top reason) with critical thinking and retention following (19\% and $18 \%$, respectively). In terms of major barriers identified in group discussions, 5 groups identified topics related to institutional support/faculty development, 4 identified incentive misalignment, and 4 cited time.
Conclusions Results suggest medical educators are intrinsically motivated to use ALMs to promote engagement, critical thinking, and retention, but perceive major barriers to successful transition, including insufficient institutional support, external incentive misalignment, and competing time demands. Given these issues generalize to all medical schools, sharing this data can clarify faculty needs to facilitate more successful program transitions.

\section{CURRICULUM}

Poster: 214

The Effectiveness of ScholarRx@ as a Supplemental Resource Following Formative Assessment in Undergraduate Medical Education

Kelly Quesnelle, Jeffrey Greene, Elyssa Weigand

${ }^{1}$ Western Michigan University Homer Stryker M.D. School of Medicine, Kalamazoo, USA

Purpose Studies in undergraduate medical education point to information organization and integration as the most common student difficulties in the pre-clinical years. Formative assessments are designed to help students identify areas of weakness. Thus, there is cause to investigate the effectiveness of a supplemental resource that is focused on organizing pertinent information following formative assessment.

Goals The goal of the present study is to investigate whether high-quality supplemental curricular resources, such as ScholarRx@, can improve student satisfaction with formative assessments and student outcomes on the summative assessment. We hypothesize that high-quality, supplemental resources will improve student satisfaction with the formative assessment process, and improve summative assessment outcomes.

Methods ScholarRx@ is a medical curriculum containing singular units of instruction, termed bricks. During the Hematology and Oncology course at our medical school, students complete optional weekly formative assessments prior to the summative assessment. Following each formative assessment, ScholarRx $\odot$ bricks were provided when associated with a learning objective sampled on the formative assessment. To assess student satisfaction with the formative assessment process, students completed surveys about supplemental learning resources. Summative assessment data is also being used to measure student outcomes associated with ScholarRx@ brick use.

Results Data analysis for this study is currently ongoing but is expected to be complete by May 2019. Preliminary analysis indicates improved student satisfaction with the formative assessment process among users of ScholarRx@ bricks compared to non-users of ScholarRx@ bricks.

Conclusions This study will provide data detailing the use of supplemental curricular resources following formative assessments. It will measure results based on both student perceptions and summative assessment performance.

\section{CURRICULUM}

Poster: 215

First Steps Toward Systems-Based Integration of a Medical School Curriculum: Neuroscience and Behavior

Riddhiben Patel, Ian A. Paul, Eddie Perkins, Tarif Bakdash, Loretta Jackson, Sajani M. Tipnis

${ }^{1}$ University of Mississippi Medical Center, Jackson, USA

Purpose For the past two decades, there have been calls for medical education reform with an emphasis on systems-based teaching and early 
clinical exposure. To overcome faculty skepticism of such changes at our medical school, we opted to begin with a proof of concept by integrating the neuroscience and behavior in a pilot that began in spring 2017.

Methods The program has been running for two years. The M1 class, Neuroscience and Behavior 1 (NSB1), integrated topics from neurobiology, neurophysiology, psychiatry, and neurology. Faculty collaborated to share lecture content. Teaching modalities in NSB1 include didactic learning sessions, gross CNS labs, active learning, laboratory demonstrations, case based learning, clinician wrap ups, and clinical experiences. In the NSB2 course topics from psychiatry, neurology, neurosurgery, neuropathology and CNS pharmacology were integrated. Twenty standardized patient encounters were added to advance the clinical educational experience and provide low stakes assessments.

Results Prior to integration, the M1 curriculum consisted of a neurobiology course and an introduction to psychiatry with a standardized NBME exam at years end. In 2016 our class mean was 45.5 and in 2017 with the new NSB1 curriculum it was 47.7. The national mean for 2013-2015 was 49.6. The majority the content areas showed improvement. In NSB2 we administered a 150 item customized exam as there was no standardized NBME. This exam was designed with questions producing an average of $76 \%$ based on previous Step 1 performance. Our class mean for this exam was $77.9 \%$. Individual content scores ranged from $74.2 \%$ in neuroscience to $82.6 \%$ in mental disorders.

Conclusion Despite concerns, the outcome of out pilot has demonstrated that performance improved and student faculty satisfaction is high. Post pilot the SOM will integrate all of the M1 and M2 curriculum. Clinically relevant elements can be introduced successfully to early students to clinically engage the learners.

\section{CURRICULUM}

Poster: 216

\section{Medical Student Perceptions Concerning Interprofessional Education: Do They Change over Time?}

David W. Musick, David B. Trinkle, Richard C. Vari, R. Brock Mutcheson

\section{${ }^{1}$ Virginia Tech Carilion School of Medicine, Roanoke, USA}

Purpose The integration of interprofessional education (IPE) competencies as early as possible into health professions education programs is a critical component of instantiating a team approach to care1. Many medical schools offer IPE content early in the curriculum, with mixed reviews by students. We sought to determine whether medical student perceptions concerning the value of IPE change over time and, if so, how such changes were reflected in student feedback and other types of educational outcome data.

Methods Anecdotally and based on years of IPE curricular experience, we observed that students perceptions concerning IPE were negative early in the medical school curriculum, but improved over time and with increasing levels of clinical experience. To explore this issue, we examined a variety of longitudinal student feedback data with emphasis on tracking the IPE experience of a single class (2017) across all four curricular years. Data sources included end of course/rotation evaluations, learning environment surveys, M3 clerkship assessment of students, residency program director surveys, AAMC graduation surveys, alumni surveys and LCME student surveys.

Results Based on exploration of the identified data, we observed an overall positive trend regarding how students perceive the value of IPE over time. Alumni survey data, collected from graduates in the midst of their residency training, were especially positive. Increased clinical experience appeared to result in a more positive view of the relevance and importance of prior IPE experiences.
Conclusion In order to evaluate the impact of IPE curricula, it is likely necessary to examine data from a variety of sources. Medical student perceptions of IPE vary across time and are likely to improve with additional clinical experience. Longitudinal cohort studies would yield a clearer picture of the perceived value of IPE to medical students and, by implication, resident physicians who have experienced IPE during medical school.

\section{CURRICULUM}

Poster: 217

\section{A New Medical Education Paradigm: Program Integration of Foundational and Clinical Sciences}

Donna DeGracia, Heather Bidinger

${ }^{1}$ MPAS Program

St. Catherine University, Saint Paul, USA

Purpose Medical education recognizes value in integrating foundational science and clinical science in new ways in order to provide students with the necessary tools for astute critical thinking and life-long learning. Evidence from around the world strongly suggests that integration is the preferred method of preparing students for current clinical practice expectations. Transition from traditional curriculum to integration is difficult for programs with limited time to deliver extensive mandated content. This presentation will introduce participants to an integrated curriculum, presenting students with a broad learning experience, bringing knowledge of foundational science to life and honing their critical thinking and analytical skills.

Methods St. Catherine University's Masters of Physician Assistant Studies (MPAS) Program, a 28-month program divided equally between didactic and clinical training, incorporates a longitudinal approach to integrating foundational and clinical sciences, understanding that clinical readiness necessitates understanding of basic sciences in connection with clinical problem solving. The curriculum utilizes multiple teaching methods and spiraling complexity to prepare students. A variety of assessment methods test critical thinking skills and ability to apply scientific concepts to clinical scenarios.

Results Preceptor evaluations, employer surveys, alumni surveys, and national standardized test scores confirm success. Benefits include knowledge retention, critical thinking skills and interpersonal relations, all crucial to practice readiness. Challenges remain in faculty training, collaboration, and faculty load. Necessary components include program administration and faculty buy-in, creativity, and flexibility.

Conclusion The MPAS Program has successfully employed the integrated curriculum since 2012. This approach seems intuitive from a clinical perspective. Our experience has convinced us that integration of foundational and clinical sciences allows for longitudinal development of critical thinking skills and improves practice readiness. While integration requires forethought, the value of improved learning experience and increased clinical readiness makes the effort worthwhile.

\section{CURRICULUM}

Poster: 218

Using a Culinary Health Experience to Teach Teamwork Skills: a New Interprofessional Education Experience 
David B. Trinkle ${ }^{1}$, David W. Musick ${ }^{1}$, Renee LeClair ${ }^{1}$, Nathaniel L. Bishop $^{2}$, Cynda A. Johnson ${ }^{1}$, Joalenn Tabor ${ }^{1}$

${ }^{1}$ Virginia Tech Carilion School of Medicine, Roanoke, USA

${ }^{2}$ Jefferson College of Health Sciences, Roanoke, USA

\section{Award Nominee}

Purpose To design a new interprofessional culinary health curriculum for first year medical, nursing and physician assistant students $($ total $=135)$.

Methods As medical schools focus more on healthy communities, there has been a corresponding expansion of teaching on nutrition and healthy cooking concepts. Students in the early years of training must learn about clinical nutrition and the dietary needs of special populations. Other relevant concepts that play a role in culinary health include population health, clinical dietetics, health disparities, quality and safety, and service learning. Interprofessional team activity in a large commercial training kitchen also offers many analogies to fast paced hospital care teams. Working with two partner institutions, we launched a culinary health program that involved three components: expanding students nutrition knowledge base, team-based meal preparation and service, and community outreach. Interdisciplinary student teams worked together during two lab sessions in a commercial kitchen wherein they cooked and served five different types of meals based on special patient populations. They also completed a nutrition oriented community service project involving inner city youth, performed a nutrition self-analysis exercise and worked through clinical nutrition cases.

Results Students provided feedback on standardized course evaluation forms, rating the culinary health experience at 3.75 on a 5-point scale. Excellent suggestions for course enhancement were received. There was a range of student buy in regarding the analogy of a commercial kitchen being similar to a busy clinical environment.

Conclusion The successful implementation of a culinary health program involving students from three disciplines requires abundant logistical support. Similar programs, during either preclinical or clinical years, may be scalable and easily accomplished. Strengths included a large student base from three disciplines and the mandatory curricular requirement for the program. Limitations included a single school intervention and one year's worth of experience and student feedback data.

\section{CURRICULUM}

Poster: 219

\section{Perceptions of End of Life Training: Is There Room for Improvement?}

\section{Tracy Wunderlich-Barillas}

${ }^{1}$ Oakland University William Beaumont School of Medicine, Auburn Hills, USA

Purpose Many medical students admit to feeling inadequately prepared and uncomfortable when caring for patients in real-time end of life situations. For the past two decades, over one-third of deaths occur in the hospital setting $(\mathrm{CDC})$, which makes it imperative to provide comprehensive end of life training to students who will be charged with providing care. This exploratory research seeks to better understand how beliefs, values and different types of knowledge influence the way students learn about death and dying to offer insight into possible improvements in training.

Methods This research has been taking place for two years at an allopathic medical school. Participants (faculty and students) were recruited using purposeful and snowball sampling methods, respectively. Enrollment is ongoing. The study involves observing students and faculty in academic settings (both classroom and clinical) as well as collecting qualitative data via semi-structured interviews with faculty $(N=5)$ who provide end of life training as well as third and fourth year medical students $(N=10)$ who have experienced an end of life situation while rounding. Thus far, both preliminary observation and interview data were coded using grounded theory to determine emerging themes and concepts.

Results Preliminary results from students and faculty suggest that the most impactful ways to learn occur by observing, listening and participating in end of life situations in the clinical setting. However, this is difficult to accomplish given limited time in the curriculum and also limited occurrence of end of life experiences.

Conclusion Based upon preliminary findings, plans currently include development of more training in hospice settings, more end of life family panels and also creation of elective rotations in palliative and hospice care. In addition, more opportunities for debriefing and discussion with peers regarding end of life experiences in the clinical setting are also being planned.

\section{CURRICULUM}

Poster: 220

Development of a Product Innovation Laboratory into the Respiratory Course in the First Engineering-Integrated Medical School

Olivia Coiado ${ }^{1}$, Dipanjan Pan $^{2}$, Kashif Ahmad ${ }^{1}$

${ }^{1}$ Carle Illinois College of Medicine, University of Illinois at UrbanaChampaign, Champaign, USA

${ }^{2}$ Department of Bioengineering, University of Illinois at UrbanaChampaign, Champaign, USA

Purpose Carle Illinois College of Medicine is creating a new model for medical education that integrates engineering principles and technology into a case-driven, active learning curriculum. This novel engineeringinfused curriculum will enable the training of a new class of physicianinnovators who will design and implement transformative approaches for healthcare delivery. To attract students that can meet this challenge, the college is hosting a series of showcases that immerses prospective students in this approach. A key showcase component is a problem-based learning (PBL) module comprising a patient scenario that integrates engineering, medicine, and teamwork concepts.

Methods The PBL module was run in groups of four-students by a facilitator. After presenting the case about an injured hiker, the students were given two tasks: The first task required students to identify symptoms and list possible medical injuries for the patient. The second task required students to build a boat out of playing cards to transport the patient and other hikers (figurines) across a river to safety. The boats were tested to see if they could remain afloat while fitting four figurines. The facilitator ended the session by summarizing the learning objectives.

Results The outcome of the PBL was gauged via task completion and facilitator input. All sessions achieved the final delivery goal of constructing and testing a boat that satisfied predetermined requirements. Facilitator feedback reflected a positive impact on students understanding of (1) how Carle Illinois PBL sessions will be facilitated, (2) the collaborative nature of student team-work and research required, and 3) how engineering concepts will be integrated into medical case scenarios.

Conclusion Development of its showcase PBL is one way Carle Illinois College of Medicine is leading recruitment of a new class of medical students and inspiring them to revolutionize healthcare through designing engineering-based solutions to current and future medical problems. 


\section{CURRICULUM}

Poster: 22

\section{Mentoring from the Ground Up: Improving Mentoring Relationships by Empowering Mentees}

\author{
Marissa Hansen, Katherine Cornelius, Heather Billings \\ ${ }^{1}$ Mayo Clinic, Phoenix, USA
}

Purpose Mentorship is an important aspect of medical education at all levels, however most mentors are not provided education in how to be an effective mentor. Similarly, most mentees are not provided education or training on how to optimize a mentoring relationship for their learning and professional development (Ramani, 2006). Considering the evolving needs of our learners and intending to provide support to grow their mentoring relationships, we designed and facilitated an eight-hour program to better prepare learners to be empowered mentees as well as future mentors.

Methods We utilized the Mentoring Competency Assessment (MCA) to measure learners self-perceptions of eight competencies through a pre and post-intervention survey design (Fleming et al., 2013). We adapted evidence-based curriculum (Pfund et al., 2014) to be delivered to learners across the health professions continuum and incorporated case based discussions, frequent low-stakes formative assessments, and peer-to-peer facilitation to heighten authenticity, relevance and immediacy to the learning experiences. Session participation by program alumni allows for contextual demonstration and competency application.

Results Preliminary data demonstrates heightened perceived competency in most domains assessed. We found mentors generally rated their mentee's skills at a higher level than the mentees' perceived ratings. We also saw significant relationships in a few domains, e.g. new vs. established relationships, females vs. males, etc. Individual session evaluations are extremely positive, and learners routinely report behavioral changes as a result of these sessions.

Conclusion We have demonstrated the successful adaptation and innovative facilitation of a successful mentorship develop program for learners throughout the continuum of health professions education. We propose that several factors discussed are critical in the success of this type of curriculum. Preliminary conclusion: A structured curriculum in mentoring relationships increases mentees empowerment and readiness to lead mentoring relationships at all levels of medical education.

\section{CURRICULUM}

Poster: 222

\section{Reconnecting the Mouth to Medical Education}

Mariah Rudd, Richard Vari, Cynda Johnson

${ }^{1}$ Virginia Tech Carilion School of Medicine, Roanoke, USA

\section{Award Nominee}

Purpose The absence of oral health education in medical education has resulted in physicians who are poorly equipped to diagnosis, treat, refer and collaborate on matters of oral health and disease. This poster describes the successful integration of an oral health curriculum, with measurable outcomes, into the medical curriculum.

Methods In the first two years of the curriculum, all medical students are now required to engage in thirty-six hours of oral health education which includes a lecture presented by an international expert on oral health, labs on anatomy/oral health, PBL patients with dental diseases, lectures on oral manifestations of systemic disease, oral cancer screening, and inclusion of the oral exam into the HEENT exam. In year 3 oral health is a component of the Family Medicine Clerkship. Assessments include a HEENOT $(\mathrm{O}=$ oral $)$ exam with standardized patients, a written exam in year 2, student satisfaction of the oral health week, and a retrospective chart review comparing documentation of oral components within student $(n=100)$ and attending $(n=$ 100) HEENT exams.

Results The average score on the oral written exam has been $89 \%$. Qualitative findings from dental week evaluations have been extremely positive. The retrospective chart review revealed that students were more likely to include the documentation of periodontal tissue (students 12\%, attendings 9\%), dentition (students $46 \%$, attendings $15 \%$ ), oral cancer screening (students $49 \%$, attendings $32 \%$ ) and mucous membranes (students $60 \%$, attendings $35 \%$ ) within their HEENT examinations.

Conclusion In a partnership with Delta Dental, who provided financial resources for the curriculum, VTCSOM has completed 5 years of educating and training future physicians on the importance of oral health.

\section{CURRICULUM}

Poster: 223

\section{Curricular Innovations: Critical Thinking and Problem Solving for} Today's Medical Student

Sarah Bezek, Nadia Ismail, Joan Friedland, Lauren Larue Walker, Jennifer Christner

${ }^{1}$ Baylor College of Medicine, Houston, USA

Purpose At Baylor College of Medicine, an innovative course for first year medical students called Critical Thinking and Problem Solving (CTAPS) was established to develop critical thinking and medical problem solving skills. Students learned about cognition, metacognitive awareness, decision making and self-regulated learning, exploring concepts independently through self-directed study, while problem solving, in teams. These concepts were explicitly built into the course through integrated clinical cases with an emphasis on Anatomy.

Methods The course was developed with faculty, residents and students. The course leadership and 16 faculty facilitators received training in cognitive science and team based learning. The first term focused on selfexploration and reflection in the context of self-regulated learning, metacognitive awareness, and problem solving, individually and in teams. The second term focused on thinking like a physician and included slow versus fast thinking, assumptions, inferences, diagnostic errors and prevention, cognitive biases, ambiguity in decision making, situational awareness, and decision making in teams. Assessment included peer/selfevaluation, individual/team readiness tests and learning strategy diagnostic tools.

Results Student feedback, short answer questions and project data indicate the vast majority of students have gained new insight into their learning processes, especially metacognitive awareness and the importance of considering how they think while solving medically relevant problems. They have also expressed insight into the power of teams in making complex decisions.

Conclusions This effort will be judged by our students' depth of understanding of learning processes and the ability to think deeply and reflect wisely as they progress. A lesson learned: 1:1 and team relationships for students and faculty can begin early. With responsive faculty and student opportunity for engagement, students support the development of innovative courses. With the opportunity to voice ideas for change, students 
from this course have expressed desire to get involved in next year's course.

\section{CURRICULUM}

Poster: 224

\section{A Dedicated Leadership Module in Physician Assistant Education}

\section{Tracey Thurnes}

${ }^{1}$ Elon University, Elon NC, 27244 USA

Purpose With recent developments in healthcare, physician assistants (PAs) will need to have leadership qualities and the willingness to serve in leadership positions as graduates. Due to an already heavy curriculum, PA programs as a whole, have not consistently implemented leadership pedagogy into their curricula. This educational innovation provided four two-hour leadership sessions in the clinical phase of the PA curriculum with the hypothesis that: PA students who are exposed to leadership discussion and activities will find it to be a beneficial learning experience, students who have completed the leadership sessions will have a higher likelihood of accepting a leadership role in their career, and that students will have a better understanding of their leadership qualities after completing the leadership sessions.

Methods During the clinical phase, PA students return to campus for a total of three weeks. During those weeks, academic content is delivered to supplement students' clinical learning, which yields an opportunity to deliver leadership strategies to students. Learning and instructional objectives were formulated for the leadership. Sessions included leadership theories, characteristic traits of successful leaders, personality assessment using psychogeometrics, leadership roles currently available to physician assistants, and team-based activities using Design Thinking pedagogy. Clinical phase PA students $(n=76)$ answered a pre and post survey answering questions regarding their understanding of leadership and personal skills.

Results Seventy-six PA students (2 cohorts) have completed the clinical phase leadership module. Data suggest that students understanding of their self, values, and leadership skills improved following the module. Students also reported that they feel more confident in their ability to hold a leadership position as a PA. Conclusion An implemented leadership module for PA students increases their understanding of leadership and their confidence in personal leadership abilities.

\section{CURRICULUM}

\section{Poster: 225}

\section{Curriculum Redesign at Wayne State University School of Medicine}

Matt Jackson, Paul Walker, Rodney Braun, Robert Lasley, Sharon Ackerman, Jean Peduzzi-Nelson, Lawrence Lash, Simone Brennan, Jason Booza, Chih Chuang, Nakia Allen, Theresa Kaminski

${ }^{1}$ Wayne State School of Medicine, Detroit, USA

Purpose Wayne State University School of Medicine (WSUSOM) launched a preclinical curriculum redesign process in 2015. This was the school's first significant curriculum change in over 30 years motivated by a need to define the competencies required for a successful transition to clerkships.

Methods A data-driven segment 1 review process that considered the opinions of all major stakeholders guided the change process. A team from the newly created Office of Continuous Quality Improvement was responsible for project management with the basic science course directors' workgroup committed to a July 2018 launch date for the redesigned curriculum.

Results The new Fundamentals of the Healthy Patient curriculum comprised eight organ system units that were organized to accommodate Gross Anatomy dissection, which was a longitudinally integrated foundation of segment 1. Introduction of a First Patient thread in the 2015/ 2016 academic year utilized cadaveric dissection to reinforce team-building, self-directed learning, and the social and humanistic aspects of medicine. In recognition of the challenges that newly matriculated medical students encounter the Office of Learning and Teaching developed a twoweek Advanced Learning Skills course as our first year student's introduction to medical school. Narrative assessment was used for all small group-teaching experiences and faculty-authored multiple-choice exams were redesigned as weekly formative assessment that facilitated academic support. The customized NBME service was used to create end-of-unit summative exams used for promotion decisions.

Conclusions Upon the completion of their first year in the redesigned curriculum, WSUSOM medical students demonstrated mastery of fundamental human processes and could develop a problem list from a patient encounter. Students demonstrated the capacity to apply the principles of social and behavioral science to clinical situations. Purposeful early integration between the basic science disciplines and new courses in the clinical and social sciences was a core theme through the change process.

\section{CURRICULUM}

Poster: 226

\section{Fostering the Development of Scholars Through Collaborative Learning \& Practice}

Mariah Rudd, Sarah Parker, Shari Whicker

${ }^{1}$ Virginia Tech Carilion School of Medicine, Roanoke, USA

Background Continued advancement of high-quality health professions education research is dependent upon the development of educators who can design research with the same rigor demanded in basic science or clinical research. Our teaching academy used a social constructivist approach to develop an interdisciplinary program comprised of a health professions education research curriculum in conjunction with an internal resource supported and mentor-guided, peer-review quality education research element as the final project.

Methods Authors developed the Health professions Education Scholars (HERS) program, a 1-year, mentorship-guided program designed to facilitate participants through individual health professions education research project. A competitive selection process was established to identify program participants. A small group of experts was recruited as mentors and critical internal resources. Program curriculum included courses from the AAMC Medical Education Research Certification (MERC) program. Participants were also mentored through the design and execution of a health professions education research project.

Results Eight individuals were selected to participate in the first year of this program. Participants represented a diverse group of health professions educators from across the health system. Participants were matched with an experienced mentor and required to protect one day per month to devote to participation in the program. At the end of the year-long program, participants completed a mentor-guided project of peer-review quality and became certified through the AAMC MERC Program.

Conclusion The primary goals of the HERS program were to provide participants with instruction in health professions education research principles and practical skills to do so effectively, 
foster collaboration within health professions education research, and develop our participants as leaders in academic medicine. The authors hope that the inaugural year improved the execution of health professions education at their institution, built a coalition of skilled medical education researchers, and ultimately contributed to national and international academic research on health professions education.

\section{CURRICULUM}

Poster: 227

Use of a Customized NBME Step 1 Subject Exam for Course Assessment in the Pre-Clerkship Curriculum

Andreas Seyfang

${ }^{1}$ Morsani College of Medicine, Department of Molecular Medicine, University of South Florida, Tampa, FL 33612, USA

Purpose At the beginning of an organ-system-based medical school curriculum, most students are not familiar with standardized board exam-type questions that would prepare them for their USMLE Step 1 exam after the second year. Hence, the purpose was to introduce medical students from their first course in the curriculum to Step 1 questions and to use performance on these questions as part of the course assessment.

Methods A customized NBME Step 1 subject exam was assembled from retired USMLE Step 1 questions that were within the scope of the lecture material covered in this first course of the medical school curriculum. This NBME final exam counted $20 \%$ of the final course grade. Individual scores of the NBME exam were subsequently z-scored to an $85 \%$ mean with a standard deviation of $7.5 \%$ and students had to reach a passline of $70 \%$ (mean $2 \mathrm{SD}$ ) in order to pass this exam.

Results When first introducing this additional NBME subject exam in our basic science curriculum for academic year 2014-15, students performed at $7 \%$ below the national average for these NBME questions. This class performance has continuously improved to currently $3 \%$ above the national average for the NBME subject exam. Part of this improvement was due to an increased use of Step 1-type practice questions throughout the lectures and as exam preparation in this course.

Conclusions The addition of a customized NBME subject exam to the basic science curriculum from the first course in the curriculum prepares medical students from the beginning of their medical school curriculum for their first board exams.

\section{CURRICULUM}

Poster: 228

\section{A Public Health Curriculum: Making It Real}

Carol Nichols, Barbara Russell, Kathryn Martin

${ }^{1}$ Medical College of Georgia, Augusta University, Augusta, GA, 30907, USA

Award Nominee

Purpose Undergraduate medical education curricula must include medical consequences of common societal problems. We have designed and implemented an interactive, pre-clinical curriculum where students learn about the role of public health in mitigating these problems at the local, state, national, and global levels.
Methods The first year curriculum included a public health module that began with an Ebola outbreak simulation to highlight global health. Social determinants of health, the opioid epidemic, and health policy issues at the state and national levels were discussed. Students participated in a poverty simulation to learn about community health. The second-year curriculum included Disaster Day training, an overnight bus tour to rural Georgia, and a trip to the Centers for Disease Control and Prevention (CDC). During Disaster Day, students activated a mobile hospital, triaged patients, participated in airway and breathing simulations, and received Stop the Bleed training. The trip to rural Georgia exposed students to healthcare in underserved areas. During the CDC visit students explored global health, chronic disease prevention, emergency preparedness, and infectious diseases.

Results Students investigated the top health issues in GA and developed Public Service Announcements and poster presentations that addressed various aspects of these issues. Additional public health issues were explored through student-led case presentations. Evaluations of how well instructional materials/methods aided understanding of topics, the extent to which the content contributed to their development as a future physician, and overall quality of the curriculum all increased as a result of these curricular changes.

Conclusions The inclusion of horizontally and vertically integrated public health content into the pre-clinical curriculum has given students unique opportunities to participate in interactive, experiential learning sessions that highlight common societal problems and the role of public health in mitigating those problems.

\section{CURRICULUM}

Poster: 229

Utilizing Interprofessional Learners in a Novel Community Outreach Program to Foster Reflection and Professional Development

Elizabeth Painter ${ }^{1}$, Michelle Davidson ${ }^{2}$, Megan McNamara ${ }^{2}$

${ }^{1}$ Louis Stokes VA Medical CenterCase Western Reserve University School of Medicine, Cleveland, USA

${ }^{2}$ Louis Stokes VA Medical Center, Cleveland, USA

Purpose Seven Centers of Excellence in Primary Care Education (CoEPCE), established by the Veterans Affairs Office of Academic Affiliations, are implementing and testing innovative approaches for interprofessional, collaborative, patient-centered practices that provide coordinated longitudinal care. As part of this initiative, the CoEPCE at the Cleveland VA has developed a comprehensive curriculum. Including domains: Proactive Careprinciples/skills designed to prepare learners to provide culturallysensitive care, participate in shared decision making, and identify psychosocial barriers to medical care, and Real Time Real Patientrecognizing the importance of reflection to foster professional development, effective interprofessional teamwork, and promote lifelong learning. This innovation focuses on the development of a community outreach project with a reflective component.

Methods Interprofessional CoEPCE learners participated in 6 community outreach events which provided education about available VA resources and completed a condensed physical exam for the initiation of VA health/ mental health care. Upon completion of the events, learners were prompted to provide a reflection, which were shared and discussed during interdisciplinary didactics.

Results In total, twenty interprofessional learners (10 Medicine/7 Nurse Practitioner/2 Psychology/1 Social Work) participated in 6 outreach events. During these events (including rural and homeless), 575 eligible veterans expressed interest in initiating VA healthcare, and CoEPCE learners conducted 135 of those physical exams. Reflections highlighted themes of reduction in bias, the impact of interacting with patients outside 
of the clinical setting, and the importance of appreciating each patients unique context.

Conclusion These events provided an opportunity, not only for a novel training experience and opportunity for reflection, but engagement with systemsbased care, as the learners are integral to the success of these events. Through analysis of the reflections and feedback, learners have demonstrated an increased ability to involve patients in their care, engage in shared decision making, and incorporate patient context in their practice.

\section{CURRICULUM}

Poster: 230

Longitudinal Efforts in Integrative Learning and Preparing For Step 1 "Designing Of A Step Up Thread"

Yenya $\mathrm{Hu}$

${ }^{1}$ Wake Forest School of Medicine, Winston-Salem, USA

Purpose Performance on the United States Medical Licensing Examination Step 1 is a prominent factor in residency program selection. Research has investigated Step 1 preparation strategies, study behaviors and anxiety management. However, comprehensive and individualized academic monitoring and longitudinal coaching that integrate the preclinical curriculum with Step 1 preparation has not been reported.

Methods The longitudinal Step Up thread includes strategies for managing cognitive loads to improve retention and integrating foundation disciplines, such as biochemistry, into system learning, using the Step 1 review sources. Step Up also provides phase-specific, individualized and outcome-driven one-on-one coaching to all students. In addition, student performances on higher order questions throughout the curriculum are monitored and assessed.

Results Students have been receptive to the practical and personalized integration of foundation knowledge, while longitudinally preparing for Step 1. Academic monitoring and individualized coaching help students identify knowledge gaps early and consistently, using their performance data, and target those gaps with customized review resources. Question banks are then utilized to assess the effectiveness of the specific reviewed disciplines or topics and to facilitate further learning. Coaching also helps students set feasible and measurable goals, develop individualized outcome-driven learning strategies and hold students accountable. Conclusion In addition to the building and implementing of the Step Up thread, the level of the student engagement is also monitored. Our preliminary data indicates that students who actively participate in self-reflection and generate outcome-driven plans appear to perform better on Step 1. Further efforts are focused on using the preliminary data to encourage all students to be more engaged in their own learning.

\section{CURRICULUM}

Poster: 231

\section{Resilience Through Parallel Charts (1) in Physician Assistant Education}

Jill P. Grant

${ }^{1}$ Wake Forest School of Medicine of Wake Forest Baptist Medical Center, Winston-Salem, USA

Purpose This educational innovation demonstrates how learners at a physician assistant program in the Southeast engage in the creation of parallel charts to process meaningful patient encounters during their clinical training. In her book Narrative Medicine, Dr. Rita Charon describes what she tells medical learners: "...you write in the hospital chart about each of your patients...[the] patient's current complaint...results of the physical exam, laboratory findings, opinions of consultants, and the plan. If your patient dying of prostate cancer reminds you of your grandfather, who died of the disease last summer, and each time you go into the patient's room, you weep for your grandfather, you cannot write that in the hospital chart...And yet it has to be written somewhere. You write it in the Parallel Chart." (1)

Methods This innovation was piloted as part of an elective humanities curriculum, later evolving into the core curriculum; more than 200 students have participated. PA learners in the clinical phase of the curriculum create parallel charts in the form of a narrative, an essay, or a poem; students may also create visual art, photography, film, or music to describe their patient encounter. Students then share their parallel charts in facilitated small groups.

Results Qualitative feedback from PA learners illustrates the benefit of creating parallel charts for "work[ing] through some of the emotional rigor that comes with learning to work with patients who are sick and dying." Students have shared that this activity is useful for self-care, for "breaking down barriers," and for teaching the value in "having coping methods."

Conclusions Fostering resilience and self-care in medical learners and future providers is essential to reducing burnout and compassion fatigue. With primary costs centered only on space within the curriculum and faculty effort toward facilitation, this activity is both feasible and applicable to all healthcare learners and providers. (1) Reference attached.

\section{CURRICULUM}

Poster: 232

\section{Collaborative Testing in Medical Education: Student Perceptions} and Knowledge Retention

\section{Jennifer Eastwood}

${ }^{1}$ Burrell College of Osteopathic Medicine, Las Cruces, USA

\section{Award Nominee}

Purpose At BCOM, we utilize collaborative testing, in which students take exams individually, then again in small groups. Prior research shows collaborative testing enhances short-term knowledge retention. We sought to understand how students perceive the value of collaborative testing and whether collaborative testing is associated with long-term knowledge retention.

Methods Quantitative data included student exam performance during the Renal 1 course, and on a post-test delivered 8 months later in Renal 2. We tested knowledge retention for two conditions: exam review and collaborative testing. After individual testing in Renal 1, groups of 2-3 students received half of the test questions with answers to discuss and half of the questions without answers to complete. The post-test included 10 comparable questions per condition from the Renal 1 exams. Knowledge retention was determined for each student as post-test score/pre-test score, and a paired $t$ test was performed. Qualitative data included responses to prompts on students perceptions of collaborative testing and exam review collected during Renal 1. Two researchers analyzed the data using grounded theory.

Results Quantitative results showed knowledge retention to be significantly higher for the collaborative testing condition as compared to the 
exam review. Qualitative results showed that students engaged in more discussion in the collaborative testing condition and valued the teamwork skills it fosters. However, students expressed that not receiving correct answers was detrimental to their learning.

Conclusion Collaborative testing enhances retention of knowledge and may be effective for students to maintain knowledge through board exams and beyond. Our findings suggest that the discursive process fostered by answering questions collaboratively is instrumental to retaining knowledge. Our data, however, do not support students perception that receiving correct answers is critical for learning. This may support promotion of an epistemic shift for students, encouraging them to value deliberative process over correct answers.

\section{CURRICULUM}

Poster: 233

\section{Integrated Clinical Case Based Learning Modules Facilitate and Improve Meaningful, Lifelong Learning Among First Professional Medical Students}

\section{Surapaneni Krishna Mohan}

${ }^{1}$ Imam Abdulrahman Bin Faisal University, Dammam, Kingdom of Saudi Arabia

Purpose Teaching of basic science courses have been a challenge in the undergraduate medical education level due to the heavy use of didactic lectures and memorization of facts. The purpose of this study is to introduce an innovative curriculum, the Integrated Clinical Case Based Learning Modules (ICCBLM) promote the teaching of the basic science course courses in terms of clinical context.

Methods The participants $(n=150)$ were first professional medical students and were divided into two groups as the control group and the study group. The educational outcomes assessed were the marks in the written examination on the traditional Curriculum with didactic lectures and those on the innovative curriculum with ICCBLM. The participants evaluated the innovative curriculum with regard to 13 items on a 5 point Likert scale.

Results The performance of the students in the innovative curriculum was significantly improved $(P<0.001)$ compared to the performance of the students in the traditional curriculum with didactic lectures in terms of the clinical knowledge. The participants perceived positively and rated positively on the effectiveness of Integrated Clinical Case Based Learning Modules.

Conclusion The introduction of the Integrated Clinical Case Based Learning Modules, an innovative curriculum model demonstrated not only improvement in academic performance in terms of clinical knowledge but also promoted the meaningful learning by virtue of integrating the basic sciences with clinical sciences, related to a particular diseases/ clinical condition among the first professional medical students. This innovative curriculum motivated the students to improve their oral presentation skills and the students perceived that, this innovative curriculum to be a valuable learning tool that improved their reasoning, inter-personal learning skills, promoted deep understanding, and motivated them to learn the subject in clinical context, rather the rote learning.

\section{CURRICULUM}

Poster: 234

Better Educators, Better Students: the Role Of Faculty Mentorship Promotes Student Competency Assessed Through Oral Patient Case Reports

Sara Maria Jensen
Trinity Medical Sciences University, Ribishi, Saint Vincent and the Grenadines

Purpose Student clinical success is largely based on student engagement and participation in routine and novel opportunities. Competencies are well defined through medical school, residency, and practice, and achieved through self-study and hands-on training. Success of students in these efforts alone is commonplace, which overlooks a key factor "faculty mentorship and guidance." However, most clinically active physicians are ill prepared entering the role of educator. Once great students themselves seemingly does not later translate to great educator. Here, we pursued how students achieve competencies through oral patient case reports with active strong faculty mentorship. We also studied if creating opportunities for student-faculty engagement improved faculty as educators.

Methods Students completing their surgical core rotation each presented one patient case report before an audience of their peers. Patients were those encountered during rotation under physician supervision. Preparation of these reports included hospital faculty advisement. Faculty included physicians that engage with students daily through their training. Before and after selfassessments were completed by students and faculty scoring aspects of competency. Faculty competencies include those under categories of: Leadership, Teaching, and Research.

Results Student competency assessed through pre/post selfassessments had larger score improvements than those from previous student presenting sessions without strong faculty guidance. On average, physicians also observed large score gaps from before and after attributed to increased (1) self-awareness and (2) clear educator objectives.

Conclusion In its entirety, success of one student requires a twofold foundation: (1) student and (2) faculty competency. The majority of students claimed improved self-performance from increased interaction and guidance from faculty based on selfassessment scores. Faculty post-self-assessment scores were higher than pre-self-assessment. Faculty comments reported heightened awareness of their own skills not consciously practiced prior to this project.

\section{INSTRUCTIONAL METHODS}

Poster: 300

\section{Medical Education Systems Worldwide: an IFMSA Report}

Marouane Amzil, Artur Carvalho

${ }^{1}$ International Federation of Medical Students' Associations (IFMSA), Copenhagen, Denmark

Abstract

Purpose The International Federation of Medical Students' Associations (IFMSA) has had a long history in meaningfully involving medical students worldwide within their respective medical education systems. Understanding its structure and physiology on a global scale was the motive to identify the main challenges and stakes in Medical Education and to develop global strategies to solve them.

Methods IFMSA conducted an online Google Form-based survey between March-May 2018 to explore how Medical Education Systems are formulated and to understand the diversity of medical curricula globally. Results Three hundred seventy-one responses were collected, covering medical schools from 83 countries. The majority (72.2\%) belong to the public sector. When it comes to curricula duration, most medical schools 
(65\%) follow a 6-year program. Basic sciences are represented in $90 \%$ of medical schools preclinical curricula, whose teaching methodologies are mainly traditional courses (77.1\%). As for the clinical training, $97 \%$ of subjects were mandatory, and use a combination of evaluation methods (written, oral and practical exams) to assess medical students in at least $29 \%$ of cases.

Conclusions The survey enabled us to highlight some medical education issues medical schools and students are facing, such as how traditional teaching courses dominate the teaching methodologies and practical skills are not adequately assessed. This survey is not meant to assess the quality of the educational institutions but rather to create a basis to discuss the various medical education systems. Understanding Medical Education Systems is an essential step towards developing a global approach to engaging medical students worldwide in their respective medical education systems, through a meaningful participation in developing their curricula. IFMSA has produced a detailed report to thoroughly present an overview of medical education systems worldwide, as a ground to develop its medical education approach to its global priorities.

\section{INSTRUCTIONAL METHODS}

Poster: 301

Influence of Graded Audience Response Questions on Lecture Attendance and Student Performance in the Pre-clinical Coursework at a Regional Medical School Campus

\section{Jeffery Fritz, Roy Long}

${ }^{1}$ Medical College of Wisconsin-Central Wisconsin CampusWausau, USA

Purpose To examine the influence of graded audience-response questions given during lectures on lecture attendance and learner performance in the second-year basic science medical school courses.

Methods This on-going two-year study is occurring at a regional medical school campus with 20-25 medical students in each cohort. All lectures during the second-year basic science courses are delivered using distance learning technology in live-streaming and recorded video formats. In one study cohort, audience response questions answered correctly during the live-streaming lecture timeframe were credited toward final grades in each course. In the other cohort, audience response questions were not graded. All other course aspects were similar between study cohorts. For each study cohort, learners have the option to (1) attend the livestreamed video lectures in a classroom setting providing real-time feedback with course instructors; (2) watch live-streamed video at a location of their preference; or (3) watch the lectures at a time and location of their choice (recorded). Learner attendance was measured as either attending lecture in a live-streamed format (comparable to in-class attendance in a traditional lecture setting) or in recorded format. Learner performance was based on final grades and summative exam performance in each course.

Results Increased usage of live-streaming video was observed in the learner cohort with graded audience response questions, however, minimal difference was observed in learner performance between the two study cohorts.

Conclusion The use of graded audience-response questions during lecture increased live lecture attendance but the increases in learner attendance did not translate into improved learner performance on summative exams.

\section{INSTRUCTIONAL METHODS}

Poster: 302

\section{TMET: a Peer-Led Medical Education Workshop}

Silvia Matilda Astefanei, Obada Yousef Al-Nahawi, Muhammad Nouman Shuja

${ }^{1}$ International Federation of Medical Students' Associations (IFMSA), Copenhagen, Denmark

Purpose Medical education should be at the core of each medical students interests, as it defines our practice. The International Federation of Medical Students Associations (IFMSA) aims to raise awareness about this matter, where its Standing Committee on Medical Education (SCOME) implemented TMET - Training Medical Education Trainers so students can acquire the necessary theoretical knowledge in approaching different issues in this field.

Methods The first TMET took place in 2014. Each workshop has a set of 2-4 facilitators who are responsible for developing its content and have had previous training on the topic. The training takes place in 3-4 days, with a maximum workload of 24 hours in total and with no more than 25 participants attending. Participants explore concepts about Advocacy, Social Accountability, Medical Students Involvement, Assessment \& Evaluation, Curriculum Development among others. Participants are required to perform a graduation training no later than 6 months after their forming took place, so they can harness their brand-new skills at their fullest, keeping themselves updated within this area.

Results Since 2014, more than 40 TMET workshops have taken place, with more than 500 trainers specialized in medical education. Trainers represent all regions of the world, with the majority of them from the Eastern Mediterranean Region and Europe, followed by Africa, Americas, and Asia-Pacific. Tunisia, Portugal, Turkey, Sudan, Ecuador, and Taiwan are the countries with the highest number of medical education trainers, all of them being TMET hosts at some point throughout this period of time.

Conclusions Engaging students to take part in this type of event not only makes them better future physicians, but it also empowers them to take proper actions in their faculties or countries and to improve the medical systems.

\section{INSTRUCTIONAL METHODS}

Poster: 303

Using Role-Play in Radiology Elective Clerkship to Enhance Medical Students Understanding of Health Economics

Thipsumon Tangsiwong, Pornpimon Kasemsook, Tipsuda Tangsriwong

${ }^{1}$ Buddhachinaraj Medical Education Center, Phitsanulok 65000, Thailand

Purpose The prospect of reducing such healthcare costs is challenging especially at a time of rising public expectations, soaring medical costs, and shrinking budgets. Practitioners are expected to have a working knowledge of health economics to better use resources and improve outcomes. This study aims to evaluate medical students understanding of health economics through experimental role-play.

Methods Twenty fifth-year medical students from Buddhachinaraj hospital who attended a three-week radiology elective in academic year 2018 were piloted in a simulation-based scenario of health economics by using role-play. This activity was supervised over a two-hour class period by instructors. Meanwhile, participants undertook their random characters as a patient, relatives, doctors and observers. During the whole session, the participants not only acted out but also took part in group discussion which they focused on investigation for reaching a final diagnosis. Then, the observers shared economic-related information which was individually assigned to prepare in advance with each other. All of them remade a consensus of the appropriate investigation based upon evidence- 
based medicine, cost-effectiveness and patient safety after that. Items and costs of the first and final-deciding investigations were analyzed eventually. At the end of the session, students' open-structural reflections were collected.

Results Achieving knowledge of health economics during in-class discussion helped students make better informed medical decisions. Accordingly, four items out of nine which the students listed earlier were identified as superfluous investigations and the final cost was subsequently reduced by over $1200 \%$. After session, the students appreciated overwhelmingly positive feedback in teaching method, facilitators and health economics as well.

Conclusion Role-play enables medical students to apply and understand economics in realistic clinical practice in order to enhance their professionalism. This initiative provides an added dimension to teaching and making health economics more clinical relevant, thereby better preparing undergraduate medical students for the real world.

\section{INSTRUCTIONAL METHODS}

Poster: 304

\section{Medical Student Participation in a Student-Led Grand Rounds Program: a Pilot Study}

Elise Zhao, Brian Piper, Diana Callender, John Szarek

${ }^{1}$ Geisinger Common, wealth School of Medicine, Scranton, USA

Purpose Peer teaching is used in health professions education but the effectiveness of student-led grand rounds as a modality has rarely been described. Focus groups revealed that our third-year medical students wanted to improve their presentation skills before starting fourth-year electives, where patient presentations in front of a group are expected. Our purpose was to develop an extracurricular program in which thirdyear medical students present their clinical encounters to students and faculty, and obtain peer feedback.

Methods The IRB-approved pilot was run in the spring semester 2018. Volunteer presenters created 20-minute presentations consisting of a patient case and background information. Review of the disease state was included for the benefit of students preparing for USMLE Step examinations. Although the program was student-led, faculty members reviewed the presentations for accuracy. Presentation criteria were rated anonymously by audience members on a scale of 1-5 (highest) and written feedback also was provided. The average scores and feedback were given to the presenters together with a survey to complete regarding their experience.

Results Eleven students gave 10 presentations and 34 audience responses were recorded. The average of all evaluated criteria was $4.5 \pm 0.08$. Twenty comments received were overwhelmingly positive. Two representative comments were Very well put together! I liked the interactive session. A little advanced for non-3rd years but something to look forward to when we get there. And, Great incorporation of cardiology, obgyn, and physiology. Great use of images to illustrate surgical techniques and physiology! Presenter was very knowledgeable! Two presenters who completed the presenter survey recommended the program to future students.

Conclusion Student-led grand rounds not only benefit third-year medical students in providing practice in patient presentations but also provides an opportunity for preclinical students to interact with third-year students aiding their board review and planning for their own clinical years.

\section{INSTRUCTIONAL METHODS}

Poster: 305
Team-Based Learning Versus Lecture: a Year to Year Comparative Analysis

Jessica Morgan Jones

${ }^{1}$ Georgetown University, Washington, DC, USA

Purpose Many medical schools have introduced learner-centered and flipped classroom pedagogical techniques such as team based learning (TBL) and decreased their emphasis on lecture as a mean of delivering content. TBL is a structured technique which includes (1) advanced preparation, (2) a short readiness assessment quiz, and (3) a group activity requiring application of concepts, often to medical cases. I investigated performance on the same material delivered either as lecture or TBL in two consecutive cohorts of first year medical students to examine the techniques effectiveness in the context of our curriculum.

Methods In year one, students received a lecture followed by a TBL exercise covering the topics of thermodynamics/pH/blood buffering and enzyme kinetics/catalysis/cooperativity, while in year two the lectures on these topics were eliminated and the material was covered solely via TBL. As a control, students received lecture alone both years on the topics of carbohydrate and protein structure and TBL alone both years on the topic of genetic inheritance. A comparative analysis of performance on identical exam items was conducted on each of these subjects. A qualitative analysis of student feedback was also performed.

Results The performance on identical test items was very similar on the two years we examined, suggesting that there was no loss of learning associated with the elimination of lectures for these topics. The qualitative analysis revealed that some students were unsure about their ability to learn in the TBL format and many stated a preference lecture. However, students also recognized benefits of working with their peers.

Conclusion We conclude that TBL can be introduced with no loss of efficacy in terms of learning, and students can also achieve non-knowledge competencies such as teamwork. However, some students are resistant to TBL and may lack confidence in their ability to learn in that format.

\section{INSTRUCTIONAL METHODS}

Poster: 306

\section{Debate as an Adjunct Tool in Teaching Undergraduate Dental Students}

Abubaker S Qutieshat ${ }^{1}$, Tala N Maragha ${ }^{2}$, Mohammad O Abusamak ${ }^{2}$, Omar Rajjoub Eldik ${ }^{2}$

${ }^{1}$ Conservative Dentistry Department, Faculty of Dentistry, Jordan Uni of Sci and TechIrbid, Jordan

${ }^{2}$ BDS student, Faculty of Dentistry, Jordan Uinversity of Sci and TechIrbid, Jordan

Purpose This work is an attempt to explore the potential of a new educational method that integrates debate as a teaching tool.

Methods This new teaching method was implemented into a single academic semester of an undergraduate bachelor of dentistry program. We sought to combine knowledge delivery with high-level debate between students, and with the active participation of the audience who were themselves students. The teaching method was introduced as an adjunct tool in the Conservative Dentistry course for fourth year dental students. The class was comprised of 281 students participating in this 10 -week teaching method that comprised of three elements namely debates, reply speeches and policy papers. Debating followed the World School Debate Championship (WSDC) format. Debate evaluation involved five main categories; argument, rebuttal, style, definition and strategy, and points of information (POIs). Student perception toward this educational method was evaluated using a Likert-scale questionnaire. 
Results Students $(N=281)$ gained valuable knowledge from the experience as reflected by the favorable Likert outcome scores.

Conclusion This work sheds light on the potential advantages of utilizing debate as a teaching tool from a student's perspective. Implementing debate in teaching improved students' ability to search and appraise scientific papers and proved to be meaningful and enjoyable. The carefully tailored 10-week format ensured good student responsiveness and engaged the entire class in an interactive, participatory setup. This educational method proved to be very effective in evidence-based dentistry teaching by promoting a deeper approach to learning that can help students to learn meaningfully and enhance their critical thinking skills.

\section{INSTRUCTIONAL METHODS}

Poster: 307

\section{Role of Service Learning in Medical Students Acquisition of Entrustable Professional Activities}

\author{
Kathryn J. Dolan \\ ${ }^{1}$ University of North Texas Health Science CenterFort Worth, USA
}

Purpose Service learning is designed to provide students with community based experiential learning. Traditionally these opportunities are thought to be supportive of altruistic behavior and development of emotional intelligence. Now they can also support the development of the AAMCs Entrustable Professional Activities (EPAs). This study aims to identify which EPAS can be supported by service learning opportunities. This study describes students' self-reported findings regarding the value of service activities in terms of four of the thirteen EPAs: 1. Gather a history and perform a physical exam, 6. Provide an oral presentation of a clinical encounter, 7. Apply clinical epidemiology to basic preventive care, 9. Collaborate with interprofessional teams.

Methods Osteopathic medical students perform service during the first two years as a required element of their doctoring course. Service learning meets various learning objectives, including exercising skills for EPAs 1, 6,7 , and 9. Students rate each service activity for learning objectives which include exercising clinical skills, preventive health education, and working in collaborative teams. This study uses 7 semesters of data which is analyzed for significant differences using both parametric and non-parametric methods.

Results Initial results demonstrate which services meet learning objectives for which EPAs: For EPA 1, services rated the highest as meeting the clinical skills learning objective are homeless services (94.3\% strongly agree) and school and sports physicals ( $81.3 \%$ strongly agree). EPA 6, homeless services (88.4\% strongly agree); EPA 7, indigent clinics (54.7\%) and sporting events (61.3\%), For EPA 9, services rated highest for meeting the collaboration objective are health fairs ( $94.5 \%$ strongly agree).

Conclusions Discernable differences exist between types of activities meeting various learning objectives and furthermore ratings ran in expected directions. Students' comments offer insights into their professional values and empathy.

\section{INSTRUCTIONAL METHODS}

Poster: 308

\section{A Student-Sponsored, Competency-Based Series of Talks on the History of Medicine}

Lindsey Kent, Peter Ward

${ }^{1}$ West Virginia School of Osteopathic Medicine, Lewisburg, USA

Purpose The humanities have been incorporated into medical school curricula to increase the breadth of student learning and to assess clinical competency beyond medical knowledge. Medical history is a topic that can reinforce learners' medical knowledge while bringing attention to social, political, cultural, and spiritual influences on its development. To explore how we could integrate medical history into a dense medical curriculum, we piloted a series of medical history talks were hosted by student clubs.

Methods In conjunction with student clubs, we crafted a series of lunchtime sessions that explored the development of medicine. Sessions covered the history of medicine from ancient times, concepts of disease, diagnosis and treatment, surgery, and the history of excluded groups. Topics involve multiple clinical competencies (patient care, professionalism, systems-based practice, etc.) and how these competencies were present or ignored throughout medical history.

Results In 2017, 205 people attended at least one session and 105 people attended all six sessions, receiving a certificate of completion. Feedback was overwhelmingly positive. In 2018, students who wished to be recognized for extra engagement wrote short critical papers some aspect of medical history and how it related to clinical competence. These papers were critiqued and returned to the students for modification prior to being considered complete. Thereafter students were able to add them to their student portfolios.

Conclusion Medical history has a tremendous potential to impact student motivation and to provide a nuanced view of how social factors impact the practice of medicine. Until these topics have a home in the medical school curriculum, it may be helpful for educators and students to explore venues such as student club meetings, to bring medical history into medical education. Giving recognition to attendees increases their engagement in the process.

\section{INSTRUCTIONAL METHODS}

Poster: 309

The Development and Use of Clinically Relevant Learning Activities in a Human Anatomy Course

John P. McNamara, Michael F. Nolan

${ }^{1}$ Virginia Tech Carilion School of Medicine, Roanoke, USA

Purpose Courses in human anatomy typically consist of lectures by faculty, readings in assigned textbooks and sometimes dissection exercises with cadavers. However, as students progress into the more clinically oriented phases of the curriculum, many discover that the anatomical knowledge they need is somewhat different from the kind they possess. We developed a series of applied anatomy learning activities structured around the general physical examination to help students appreciate the importance of anatomy in the evaluation and management of patients. Methods We developed seven applied anatomy laboratory sessions with content appropriate to each of three, regionally organized blocks in our anatomy curriculum: limbs and back, thorax and abdomen. The exercises involve inspection, palpation and other maneuvers commonly included in the general physical examination. Students are divided into small groups and instructed to perform each exercise on all other members of the group. Faculty circulate among the groups to guide students in the performance of tasks and to answer questions as they arise. Short written questions accompany many of the exercises that highlight anatomic structures and relationships associated with the body part or region under study. Students were surveyed at the end of each unit regarding their perception of the value of these exercises.

Results Students generally liked the active learning format of these sessions, particularly their close relationship to the clinical skills they were learning concurrently in other courses. Students appreciated the fill in the blank questions which they viewed as practice questions for the upcoming final exams. Some students felt somewhat uncomfortable serving as 
subjects for their peers. This hesitancy gradually diminished as the year progressed.

Conclusion Students acknowledged the value of these active learning activities early in the curriculum as directly related to essential knowledge and skill sets that will be necessary in the clerkship and elective years.

\section{INSTRUCTIONAL METHODS}

Poster: 310

Training Improves Student Performance and Perceptions in Small Group Learning

\author{
Mari K Hopper \\ ${ }^{1}$ Indiana University School of Medicine, Indianapolis, USA
}

\section{Award Nominee}

Purpose Fifty percent of course contact time in Indiana University School of Medicines (IUSM) renewed curriculum was dedicated to small-group learning. Course evaluations indicated that students did not value the approach, perhaps due to lack of training in this methodology. This study set out to determine if training in small group dynamics would improve student participation in and perception of this learning method.

Methods Following IRB approval, case-based small-group sessions were audiotaped on two occasions prior to training (Pre), and two sessions following training (Post). Recordings were evaluated and scored by trained evaluators using a rubric including the following categories: Participation, Shared Roles, Focus on Learning Objectives, Approach, and Interpersonal Interaction. Category scores were averaged across the three evaluators both Pre and Post. To assess student perceptions, a 15question survey was administered at three time periods: (1) before Pre small group sessions; (2) after recording Pre sessions and directly prior to training; and (3) after Post small group sessions. Topics such as personal preparation, interpersonal interactions, and prior undergraduate experience were included in the survey and rated using a Likert scale scoring one through seven.

Results Although work is ongoing, preliminary analysis of audio recordings indicate that participation increased following training, with members participating more equally and encouraging input from others more frequently. There was little change noted in role sharing and addressing learning objectives. Survey responses following training revealed that students enjoyed small group sessions more, contributed more equally, and had fewer tangential discussions when compared to pre-training. Conclusion These data indicate that training in small group dynamics prior to participation in small group learning activities will improve student discussion performance and perceptions of the learning experience.

\section{INSTRUCTIONAL METHODS}

Poster: 31

\section{From the Basic Concept of ph to Complex Acid-Base Disorders in Two Hours Using Self-Directed Learning}

Michael Bradbury ${ }^{1}$, Mark Sheffield ${ }^{2}$

${ }^{1}$ Kirksville College of Osteopathic Medicine, A. T. Still University, Kirksville, USA

${ }^{2}$ California Northstate University College of Medicine, Elk Grove, USA

Purpose Self-directed learning (SDL) can aid in the development of life-long learning skills. Implementation of SDL is new to many, and methods used and topic chosen may affect successful implementation. As many students struggle with acid-base concepts, a session on basic concepts and clinical relevance was designed and presented. The session and the outcomes are described.
Methods The session was adapted from the system developed at Albert Einstein College of Medicine. A basic scientist and clinician each developed 3 learning objectives for their respective areas. Students were provided with objectives in advance and assigned to work in groups. Students were to find resources to learn the material and be prepared to work with it during the session. In the session student groups answered basic science questions or performed calculations in front of the class. Later in the session clinical cases were presented which groups were asked to expand upon. If answers were incomplete, a second group was chosen to add information. After the session, they had 24 hours to submit their learning resources documents. Each submission was reviewed and feedback was given.

Results The session was attended by the entire class and all groups participated. Students worked through both the basic and clinical problems presented, though completeness of answers varied. The students remained engaged throughout. All groups submitted resource documents on time and received feedback on their choice and evaluation of sources. Exam performance on the material was satisfactory.

Conclusion Combining basic and clinical information in SDL format is possible and likely desirable. Students can complete an SDL task if given appropriate instruction on expectations. Student choice of resource material varies widely as does their evaluation of its utility. Material presented appeared to be learned based on exam results, so the exercise was deemed successful as a first effort.

\section{INSTRUCTIONAL METHODS}

Poster: 312

An Interprofessional Experience of Bioethics for Health Professions Graduate Students: the Ethics Health Care Team Challenge

David W. Musick, David B. Trinkle, Phyllis B. Whitehead, Chris Pile, Joalenn Tabor

${ }^{1}$ Virginia Tech Carilion School of Medicine, Roanoke, USA

Purpose To expose medical, nursing, occupational therapy and physician assistant students to collaborative, case-based decision making featuring ethical dilemmas associated with patient care.

Methods The health care team challenge is an innovative teaching method used in a variety of health professions education settings. The ability to resolve complex ethical dilemmas associated with patient care is an important part of health professions education. The opportunity to expose students to this training in an interprofessional context and to allow them to see a hospital ethics consultation panel at work is an innovative curricular approach. After an interprofessional course introducing bioethical principles to approximately 135 health professions graduate students, the entire student body was assembled for a cumulative Ethics Health Care Team Challenge event. After initial presentation of a clinical case, student teams were formed and defended an assigned ethical position related to a patient case featuring ethical dilemmas. The ethics consultation panel from our teaching hospital subsequently listened to student presentations and led further discussion of the case, ensuring that all spheres of ethical care (clinical, psychosocial, spiritual, legal and financial) were explored. At the conclusion of the panel discussion, the actual events and ultimate resolution of the case were presented.

Results Over three consecutive years, student feedback was uniformly positive. Changes were made each year to better address nuances associated with student group activities, and to accommodate online learning by distance students.

Conclusion The health care team challenge format is adaptable and generalizable to a variety of instructional settings, and is low cost in terms of resources needed. This learning format combines several teaching methods, yet is relatively compact and easy to reproduce. Limitations include technology availability and length of the event $(3 \mathrm{~h})$. Special care must be taken in selecting an appropriate clinical case which features ethical complexity. 


\section{INSTRUCTIONAL METHODS}

Poster: 313

\section{Medical Nutrition Therapy Immersion to Enhance Learning and Use of Nutrition Counseling}

\author{
Aaron Marshall, Heather Christensen \\ ${ }^{1}$ University of Cincinnati, Cincinnati, USA
}

Purpose Nutrition as a component of patient care is widely recognized for contributing to optimal clinical outcomes. Despite increased efforts to teach nutrition basics to trainees, nutrition counseling remains woefully underutilized. This study assessed the impact of a medical nutrition therapy (MNT) experience on medical students. The ultimate goal is to provide a personal experience that would equip trainees with tools to increase their use of nutrition counseling.

Methods Participants voluntarily adhered to an MNT diet for two weeks. At three timepoints, participants completed a Nutrition in Patient Care Survey (NIPS), which is a reliable measure of attitudes about nutrition in patient care. Data from the 45 multiple-choice questions within the NIPS are grouped by subscales: nutrition in routine care (NRC), clinical behavior (CB), physician-patient relationship (PPR), patient behavior/ motivation (PBM) and physician efficacy (PE). To determine the use of nutrition counseling by medical students, we video-recorded and evaluated (using a validated rubric) clinical encounters between students (in groups of four) and standardized patients.

Results Results of the initial NIPS data $(n=28)$ yielded the following subscale scores [average (SD)]: $\mathrm{NRC}=33.82(3.98), \mathrm{CB}=22.11(1.95)$, $\mathrm{PPR}=35.25(2.56), \mathrm{PBM}=10.64$ (2.39), and $\mathrm{PE}=19.46$ (3.68) (internal test reliability confirmed by Cronbachs alpha $>0.6$ ). Qualitative baseline data show that, prior to the MNT experience, the mention of nutrition in a standardized patient encounter is similar between student groups that include MNT participants and those that do not (complete data set available by conference date).

Conclusions NIPS results indicate that study participants have similar attitudes and expectations about nutrition in medicine as those reported in other published cohorts. Initial scores from the NIPS Likert-scale questions allow room for differences to be identified, should the MNT experience alter participant attitudes. Preliminary data from clinical encounters indicates that a bias toward nutrition does not exist in the MNT participants.

\section{INSTRUCTIONAL METHODS}

Poster: 314

The Use of the 5E Instructional Model to Teach Respiratory Medical Physiology

David M Harris, Benjamin Daines, Andrea Berry, Atsusi Hirumi

${ }^{1}$ University of Central Florida, Orlando, USA

Purpose Although there are trends in medical education to increase active learning, there is limited literature on the use of learner-centered instructional strategies to develop curriculum material. We used the $5 \mathrm{E}$ (Engagement, Exploration, Explanation, Elaboration, Evaluation) instructional model to scaffold 13 hours of contact time to teach respiratory physiology.

Methods One hundred twenty first year medical students participated in this study. For the Engagement phase students were introduced to a family with multiple respiratory conditions. The instructor provided resources, learning goals and expectations during this phase. During the Exploration phase, students were given calendar time to produce 4 mechanism of disease (MOD) maps individually and submit. During the Explanation phase, students worked in groups and formulated a group
MOD map for each case based on negotiated understanding. The Elaboration phase occurred the following week where student groups generated MOD maps for more complex cases. The Evaluation phase occurred across the other phases. Faculty served as facilitators during each phase as they went group by group and also were responsible for providing feedback in a debrief style setting where a group presented their MOD map to other groups.

Results A post experience survey was distributed to determine the strengths and weaknesses of the pedagogical approach. Positive comments included thinking with groups or critically. Negative comments revolved around lack of a faculty version of the MOD maps. Pretest questions given before instruction will be compared with summative exam questions covering frequent misconceptions in respiratory physiology. Individual and group MOD maps will be analyzed for conceptual understanding.

Lessons learned

Help from an instructional designer was crucial for this project. Instructors will face some criticism because learners have a different perspective of what makes learning "efficient."

\section{INSTRUCTIONAL METHODS}

Poster: 315 A Collaborative Learning Workshop to Facilitate Cognitive
Integration in Undergraduate Nursing Students

Jeanette Ignacio, Hui-Chen Chen

${ }^{1}$ National University of Singapore, Singapore, Singapore

Purpose Undergraduate nursing education is consists of basic science knowledge that underpin the practice of patient management skills and clinical decision-making. Students are expected to link what they've learned from different modules in their curriculum. This integration, however, does not readily take place. A collaborative learning workshop was conceptualized to facilitate consolidation and integration students learning at the end of the semester.

Methods A collaborative learning workshop was developed for Year 1 undergraduate nursing students enrolled in the following modules: Comprehensive Health Assessment, Pathophysiology \& Pharmacology, and Medical-Surgical Nursing. In this activity, all students in these three modules were given materials for pre-reading prior to the workshop. During the workshop, the students gathered in a big lecture theater where they were presented with a clinical case study. After which, the students were split into small groups and discussed the case study in their own tutorial rooms. A faculty was assigned to each group to facilitate the discussion. After an hour, all the groups went back to the lecture theater where selected members of each group applied what they have discussed as they managed a standardised patient (SP) simulating the patient in the clinical case study they have discussed. The rest of the students observed. Feedback and consolidation were then done after the students have managed the SP to conclude the session. Focus group interviews were conducted after the students to evaluate the value of the workshop to their learning.

Results Fourteen students participated in focus group discussions. Themes that were generated included (1) having the correct mindset; (2) building-up confidence; and (3) tutor expertise.

Conclusion The collaborative learning workshop is a promising strategy to facilitate cognitive integration that prepares undergraduate nursing students for their clinical placements. Refinements to the workshop are needed to optimize its benefits to students learning.

\section{INSTRUCTIONAL METHODS}

Poster: 316 
Instruction Delivered from Practicing Medical Professionals Shows Positive Outcome in Interprofessional Pre-health Profession Undergraduate Students

Brian J. Robinson, Sarah J. Garvick

${ }^{1}$ Physician Assistant Studies, Wake Forest School of Medicine, WinstonSalem, USA

Purpose There is limited literature on pre-health professional undergraduate students' preference to be instructed by practicing medical professionals or by non-practicing full-time faculty. The objective of this evaluation was to determine student experience of practicing medical professionals in delivering an effective interprofessional course.

Methods Fifteen pre-professional, undergraduate students selected to enroll in an optional five week online and classroom hybrid course on interprofessional education that utilized web-based training as well as face-to-face sessions taught by clinically practicing physician assistants. The online training consisted of videos, discussion boards, and journal article reading. The live sessions consisted of small group learning, lectures, case-based activities, and panel discussions. Enrolled students completed pre- and post-surveys. Descriptive statistics were used to analyze the data.

Results All ( $n=15,100 \%)$ students felt that having health professionals teach this course was beneficial to understanding and application of course material, beneficial to take prior to applying to their professional field, and would recommend this course to peers. All $(n=15,100 \%)$ students reported improvement in one or more of the following categories: communication, patient centered care, teamwork, and understanding of other health professions. A majority of students $(n=11,73 \%)$ commented in addition that professionals teaching the course improved the course experience and that the panel discussion at the end of the course involving clinicians from multiple healthcare fields was insightful. Four students $(n=4,27 \%)$ thought that increasing the amount of face-toface time with healthcare professionals, opposed to online content, would improve the class overall.

Conclusion Despite limited face-to-face instruction, students enjoyed this course and demonstrated improvements in all core interprofessional competencies. Future improvements to the course include increasing face-toface time between students and clinicians. Future studies should include a larger cohort of students and address the noted preference for face-to-face discussion versus online instruction.

\section{INSTRUCTIONAL METHODS}

Poster: 317

\section{A Health Literacy Activity to Enhance Medical Students' Communication Skills}

Kathleen J. Doane, Ying-Ju Sung, Patrick M. Boyd

${ }^{1}$ Geisinger Common wealth School of Medicine, Scranton, USA

Purpose To enhance a health literacy activity designed to aid first year medical students understanding of how to speak to patients by having them develop and analyze patient education materials.

Methods We will modify an activity designed for first year medical students (Doane and Boyd, 2016). After the case discussion, students were asked to write patient education materials to help support the patient and their family in understanding and managing the disease. Faculty analyzed these pamphlets using Suitability Assessment of Materials (Doak et al., 1996) and readability analyses available on-line. For the next iteration, students will be asked to generate a pamphlet based on new cases, and then to modify them based on analyses with these tools such that they are at an appropriate level for patients with a 5th-6th grade level of education. Students will write a brief summary prior to the activity and subsequently on how well they understand patient communication at an appropriate level.

Results In the initial activity, students generated pamphlets that were not always accessible based on the readability level. Students indicated that they understand the goal of speaking on an appropriate level with their patients, but that they are unsure if they are accomplishing this. We expect that modifying this activity by having students use the suitability and readability tools to modify their pamphlets, they will better understand how to communicate more effectively with their patients.

Conclusions Learning how to speak to patients at an appropriate level is a vital component of health literacy and patient-centered care. We predict that this activity will greatly increase our students understanding of patient communication. We plan to enhance the activity further by making this an interprofessional education activity.

\section{INSTRUCTIONAL METHODS}

Poster: 318

An Interactive Method to Facilitate Durable Learning and SelfAssessment in a Basic Medical Science Course

Michael F. Nolan, John C. McNamara

${ }^{1}$ Virginia Tech Carilion School of Medicine, Roanoke, USA

Purpose Previous research has shown that both structured feedback and retrieval practice facilitate durable learning. We have used both techniques separately in our curriculum and have now developed an activity incorporating both in a basic medical science course.

Methods We divided our class into groups of seven students. We then projected challenging, vignette type MCQs for all groups to consider. After approximately $1 \mathrm{~min}$, a randomly selected group was called upon to share their answer and explain their rationale for choosing that particular answer. Other groups were then called upon similarly. Differences in answer choices and explanations between groups were then discussed and errors suggesting incomplete or incorrect learning were identified.

Results All students appreciated the practice question format (retrieval practice). Students valued being challenged to explain their reason(s) for choosing a particular answer (effortful retrieval). Most students appreciated the inter-active discussions (peer feedback) and all students appreciated the brief explanations provided by the instructor when needed. Some students did not feel comfortable speaking in front of their peers. Some students preferred the instructor to simply speed through as many questions as possible, forgoing any discussion or clarification that might have occurred during the activity.

Conclusion To maximize the value of the sessions the questions must be sufficiently challenging to identify weaknesses in understanding. Students must defend their reasoning clearly and all students must be satisfied with the conclusions reached before moving on to the next question. Instructors must be sensitive to students who may not wish to speak and not force a student to expose their uncertainty or unfamiliarity with the material. Resist the temptation to speed through the question pool, particularly when it is evident that some students are unclear or uncertain about a particular topic.

\section{INSTRUCTIONAL METHODS}

Poster: 319

Hands-On Culinary Medicine Modules Enhance the Acquisition of Nutrition Knowledge Increasing Student Confidence in Their Ability to Counsel Patients

Kathryn H. Thompson ${ }^{1}$, Douglas B. Spicer ${ }^{1}$, Leslie A. Ouellette ${ }^{2}$, Meredith Kenneally ${ }^{2}$ 
${ }^{1}$ College of Osteopathic Medicine, University of New England, Biddeford, USA

${ }^{2}$ Westbrook College of Health Professions, University of New England, Biddeford, USA

Purpose Fifty percent of Americans live with one or more chronic illnesses. Poor diet is a risk factor for many of these diseases. Although health care professionals are uniquely positioned to help patients make healthier choices they often lack the training and conviction to deliver this guidance. Tulane University has successfully implemented a culinary medicine curriculum that combines hands-on cooking skills and food procurement with nutrition education to enable the development of a holistic approach towards counseling patients. In this study, we used two of the Tulane modules to measure the importance of the hands-on cooking activity on nutrition knowledge acquisition and retention and on attitudes towards nutrition counseling.

Methods A randomized crossover design was used for this intervention. Forty-three first-year medical school students participated in the study and were randomly assigned to two groups. During each week one of the groups participated in hands-on cooking instruction and group discussion while both groups completed the online culinary medicine modules. At the end of each week, students were tested on the weeks content. Retention was measured one week later using a similar test. Attitudes towards counseling patients were surveyed and qualitative analysis of the data was completed.

Results Analysis of the effects of the hands-on cooking activity on learning and retention will be presented and discussed. Students perceived that the activity improved retention of the material. Students commented that completion of the hands-on cooking activity gave them more confidence in applying nutrition concepts to daily life and they were more likely to discuss this with patients.

Conclusions Participation in the hands-on cooking activity helped students realize that small, easy changes in meal preparation can have a profound effect on their patients' health. The modules provided scientific background to support nutrition recommendations and students recognized the value of this for counseling patients.

\section{INSTRUCTIONAL METHODS}

Poster: 320

\section{Interprofessional Education in Ultrasound Instruction: Creating Connections Across Healthcare Disciplines}

\section{Kristin J Henderson, Ian M Smith}

${ }^{1}$ Wake Forest School of Medicine, Winston Salem, NC 27157 USA

Purpose Modern healthcare requires a variety of clinical, personal, and professional skills. Wake Forest School of Medicine exposes graduate students to interprofessional education (IPE) to improve critical thinking, preparedness, and patient safety. This abstract will discuss the 5 phases of IPE ultrasound education delivered in Nurse Anesthesia by PA faculty. Methods In 2016, CRNA faculty identified the need to implement an ultrasound curriculum. When administering peripheral nerve blocks (PNB), ultrasound guidance improves success rates and reduces complications. Previously, CRNA students received no formal ultrasound education before clinical instruction in PNB. The CRNA faculty sought to provide ultrasound instruction to improve preparedness and increase the number of PNB completed by CRNA students during their training. The faculty developed a 5-Phase process, wherein CRNA students learn ultrasound application through lecture, lab, and workshop in an IPE format. Results Since the implementation of IPE ultrasound instruction, successful PNB administration by CRNA students increased from a median of 9 (2016) to 20 (2018) (122\% increase). Students attributed this increase in PNB placement to improved preparation and understanding the procedure.
Conclusion Ultrasound IPE has improved the preparation and skill in CRNA students placing PNB. Because of this collaboration, IPE activities between CRNA and PA faculty have continued to evolve.

\section{INSTRUCTIONAL METHODS}

Poster: 321

Effectiveness of Video-Based Flipped Classroom Using CustomMade Commercial Videos for Improving Medical Physiology Teaching

Praveen Kottath Veetil

${ }^{1}$ Avalon University School of Medicine, Willemstad, Curacao

Purpose Flipped classroom techniques may improve student performance because they allow more time for active learning and formative assessment. When a custom-made commercial video is used for the video-based flipped classroom (VBFC), the hypothesis is that students may improve in scores as per the constructivism theory of learning, and may find it beneficial.

Methods In Physiology course, students are required to watch custommade commercial short (less than 10 minutes) videos followed by 1 to 5 MCQs, with facilities to monitor student watching for VBFC. The class hours are utilized more for an interactive learning followed by in-class formative assessment with feedback. Surveys were conducted pre and post-implementation. The isolated grades of Physiology exam were compared with the overall grades using the paired t-test and Physiology scores were compared between VBFC students and students with traditional lectures using unpaired $t$ test.

Results In the survey, students uniformly agreed with the benefits of this method and showed strong agreement with three aspects; duration of each video, quality, and usefulness of 1 to 5 questions that followed each video, and the need to continue this method of teaching. Some descriptive comments in the survey will help to make future improvements. Physiology scores, which was significantly $(p=0.0216)$ below the overall scores before VBFC, has improved and became equivalent to the overall scores after implementation of VBFC.

Conclusion Explained with the base of Piagets theory of constructivism, students improved Physiology scores by the process of accommodation and assimilation. Use of commercial videos helped to overcome limitations of a traditional VBFC like the need for technical skills, preparatory time, and monitoring. The limitations of this study are small population size and short study duration; before conclusive evidence, we need an elaborate study for this method of VBFC.

\section{INSTRUCTIONAL METHODS}

Poster: 322

The Integrated Illness Script: Answering the Why Question in Diagnostic Reasoning

${ }^{1}$ Amy Wilson-Delfosse, Michael Dell ${ }^{1}$, Leslie Fall ${ }^{2}$, Tracy Fulton ${ }^{3}$, David Harris ${ }^{4}$, Ann Poznanski ${ }^{5}$, Brian Wilcox ${ }^{5}$

${ }^{1}$ Case Western Reserve University School of Medicine, Cleveland, USA

${ }^{2}$ Aquifer and Geisel School of Medicine, Lebanon, USA

${ }^{3}$ San Francisco School of Medicine, University of California, San Francisco, USA

${ }^{4}$ University of Central Florida College of Medicine, Orlando, USA

${ }^{5}$ Geisinger Common wealth School of Medicine, Scranton, USA

Purpose Illness scripts are knowledge structures in the minds of clinicians that organize epidemiology, clinical findings, and pathophysiology of medical conditions and help physicians determine if a given patient 
presentation fits the pattern of typical findings for a given disease. We propose that inclusion of basic science causal mechanisms that explain why a patient manifests each clinical finding will enhance clinical reasoning. Here, we describe the development of the Integrated Illness Script (IIS) as a teaching and learning tool and document initial efforts toward building a library of exemplar IISs.

Methods Six pilot schools with teams of basic scientists, clinicians and students were recruited to optimize the process for generation of IISs that support the most common conditions required by nine national core clerkship conditions. Following agreement regarding prototypical clinical findings of each condition, mechanistic details specific to each finding were juxtaposed with each clinical finding. Mechanism of disease (MOD) maps aligned with each IIS were also generated to integrate the fundamental basic science concepts and mechanisms that underlie each IIS. Results Pilot schools have worked collaboratively to optimize and identify best practices in the creation of IISs. The use of this tool to promote clinical reasoning also has been introduced to numerous schools through national workshops and has been used in pre-clerkship and clerkship education at a number of medical schools. With minimal instruction, students are able to effectively create IISs for diagnoses they are considering as part of their clinical or case-based experiences.

Conclusions The Integrated Illness Script is an easy tool to implement to ensure the integration of basic science mechanistic understanding into the clinical reasoning process. Next steps are to continue developing a set of exemplar IISs and to continue to pilot test the impact of IISs in clinical reasoning.

\section{INSTRUCTIONAL METHODS}

Poster: 323

\section{Large Group Lecture Demonstrations to Engage First Year Medical} Students in Gross Anatomy and Neurobiology

James W Lewis, Anna Lama

${ }^{1}$ West Virginia University, Morgantown, USA

Purpose With the advent of lecture capture, face-to-face teaching in medical school large classroom settings are increasingly under pressure to transition to online teaching, flipped classes, and blended learning approaches, or suffer from lower attendance and lower attainment. This places a greater onus on lecturers to include engaging, dynamic teaching methods in large classroom settings to convey key concepts in a manner that holds value beyond their recorded substitute. The purpose of the education innovations herein were to introduce and assess several specific on-stage demonstrations that can be presented in large classroom settings and convey information beyond textbook or gross lab experiences, slated for first year medical school and nursing school curricula.

Methods Multiple demonstrations were implemented during traditional 1 hour class lecture slots, including teaching the fluidics of cerebral spinal fluid flow, the mechanics of hematoma formation, and teaching an overview of brain blood supply, among other topics. Likert scale (1-5 ratings) feedback and solicited comments were collected immediately after the course, as well as from a subsample of the students a year or more later to assess longer term retention.

Results Student evaluations and comments from medical students accumulated over three years were assessed, providing input regarding refinements and modifications over the years. In addition to content relevance to national standardized exams, feedback provided insights into time allotment to demonstrations, and size and visibility of props for large classroom settings.

Conclusion While not all demonstrations may be appropriate for any single medical curriculum course, the ideas are transferable and generalizable to other schools and curricula under different logistical teaching circumstances. The overarching goal was to foster thoughts on how to encourage, rather than mandate, classroom attendance and retain the value of face-to-face teaching and roles for teachers in a changing environment competing with the technology-as-education reform.

\section{INSTRUCTIONAL METHODS}

Poster: 324

"Nothing Operates in Isolation": Learning Biochemistry and Physiology via Drawing

Weichao Chen, Selina Noramly

University of Virginia School of Medicine, Charlottesville, USA

Purpose Despite the instructional benefits of drawing, recent literature review highlighted a need to explore strategies to effectively integrate drawing into teaching. Following four-year experimenting with different strategies, we started investigating undergraduate medical students perception of and experience with learning biochemistry and physiology via drawing.

Methods A series of sessions were offered at the onset of the pre-clerkship period teaching biochemistry through drawing, including using drawing as a review vs. preview, giving a stand-alone drawing session vs. integrating drawing with others lecturing in one session, and providing life vs. pre-recorded demonstration. Afterwards, all 156 learners were invited to provide feedback on these sessions.

Results Detailed outcomes from the theme analysis of student responses will be presented. Among the 124 students who had compared learning through drawing as a preview, integrated review, vs. stand-alone review, 123 found drawing helpful. Realizing that "nothing operates in isolation," learners appreciated getting a big picture to integrate all the metabolism pathways. Specifically, students acquired a road map in the preview to orient their subsequent learning and tested their comprehension through the reviews. Acquisition of conceptual understanding was perceived both as a prerequisite for and outcome from learning related information through visualization. Such perception contributed to mixed perceptions about how mapping should be integrated with lecturing. Among the 127 students who had indicated their preference of drawing individually, with peers, or following instructor demonstration, 103 (81\%) preferred instructor demonstration when learning new materials or more complex pathways. Learners explained their rationales of preference, discussed how they had integrated drawing into their self-study, and offered suggestions to further benefit from instructor demonstration.

Conclusions We will incorporate students' comments to enhance the design of teaching physiology with drawing, to be offered during Spring 2019. Additional data will be collected, and findings will be shared.

\section{INSTRUCTIONAL METHODS}

Poster: 325

Creating Engaging Faculty-Produced Microlecture Videos for Flipped Classroom in Undergraduate Medical Education

Jonathan Fisher, Yerko Berrocal, Leslie Hammersmith, Andrew Darr, Anthony Dwyer

${ }^{1}$ College of Medicine Peoria, University of Illinois, Peoria, Illinois 61656 USA

Purpose Many US medical schools are moving from traditional lecture-based curriculum in favor of ones that incorporate more student-directed, active learning strategies. The University of Illinois College of Medicine (UICOM) is undergoing a curricular transformation that promotes multiple active learning modalities like flipped classroom, wherein students prepare by watching microlecture videos prior to designated in-class sessions. 
Methods Topics for microlectures were identified at least 12 weeks prior to the scheduled in-class session. Faculty worked alongside instructional design specialists to develop the videos, which were recorded and edited using TechSmith Camtasia software. Faculty included custom-created animations and review questions in the videos to stimulate student engagement. Quality control was maintained through a peer review process to evaluate multiple factors, including managing segmenting, signaling and modality within the recording to ensure the videos met standards that promote student engagement. Throughout this process, the college has developed a solid base for the development and use of video microlectures as an effective tool for flipped classroom sessions.

Results UICOM faculty produced over 300 microlecture videos in the last two years. The average length of these videos is 15 minutes. The total hours of video produced is 4573 . Student feedback to the microlectures has been positive. Faculty peer review of video microlectures occurs both formally and informally as part of the regular design process.

Conclusion The microlecture format is an effective way to deliver content to medical students in preparation for flipped classroom activity, especially when authors design videos to meet standards for quality digital instructional media. UICOM continues to add to the microlecture pool and revise existing videos for incoming students.

\section{INSTRUCTIONAL METHODS}

Poster: 326

Teaching and Assessing Pharmacology Learning Using Different Approaches in UCSFs Integrated Bridges Curriculum "Omnes viae Romam ducunt?"

Marieke Kruidering-Hall, Rupa Lalchandani Tuan

${ }^{1}$ University of California, San Francisco, USA

Purpose There are many approaches to teaching pharmacology, but which approach best promotes learning and application of pharmacology knowledge is not well studied. Here, we report on student pharmacology openended question (OEQ) performance across multiple blocks that have different teaching strategies. The UCSF Bridges curriculum is an integrated organ systems based curriculum. The pre-clerkship component, Foundations 1 (F1), is 18 months in duration and consists of 10 Foundational Sciences (FS) Blocks, Clinical Microsystems Clerkship, Inquiry Curriculum, and Assessment Reflection Coaching \& Health weeks. Students learn via multiple modalities including (1) live lectures, (2) small groups, (3) online videos, (4) online PowerPoints to click through independently and (5) interactive, integrated case-based wrapup sessions. Assessments incorporate the principles of testing for learning (1) through required weekly quizzes consisting of multiple-choice questions (MCQs) and OEQs. Summative assessments are fully OEQ-based and require students to demonstrate application of knowledge in order to pass.

Methods Setting: Academic years (AY) 16-17 and 17-18. We collected medical student ( $n=152$ per year) pharmacology OEQ exam performance from the introductory first block (which utilized modalities $1 \& 2 \& 3 \& 5)$, the cardiovascular block (1\&2\&4), the renal, GI, endocrine $\&$ nutrition block ( $1 \& 2 \& 3 \& 5+$ flashcards provided on exam), the infection, inflammation and immunology block $(3 \& 5+$ flashcards provided on exam) and the neuropsychiatry block ( $3 \& 5)$. OEQs were graded using a rubric: meets expectations (score 5,6), borderline (score 3,4) or does not meet expectations (score 1,2). Pharmacology performance will be compared across blocks using repeated measures analysis of variance.

Results We are currently finalizing data collection and can guarantee that we have access to the data to undertake the analysis and present this work. Conclusions From this study, we will gain insight into which specific teaching methods, if any, are more or less effective at facilitating application of pharmacology knowledge on OEQ exams.
References

Brown, P.C. et al. (2014) Make it stick: the science of successful learning. Harvard University Press.

\section{INSTRUCTIONAL METHODS}

Poster: 327

Adapting the First-Year Integrated Curricula in Response to the Needs of Developing Students Self-Regulated Learning Skills

Lu Xu, Shana Zucker, Blake Milson, Hannah Lo

${ }^{1}$ Tulane University School of Medicine, New Orleans, USA

Purpose Medical schools aim to provide learning experiences that encourage the development of self-regulated and lifelong learning skills in medical students. However, it is unclear whether medical students become better self-regulated learners as they progress through the pre-clinical years. The objectives of this project are (1) to investigate whether the current pre-clinical year integrated curricula will improve self-regulated learning skills; (2) to investigate whether these skills are related to academic performance.

Methods We employed a longitudinal study design. The Self-Regulation of Learning Self-Report Scale (SRL-SRS), was administered to three student cohorts (2017-2020) 3 times: (1) at the beginning of first-year integrated curricula; (2) at the end of first-year integrated curricula; (3) at the end of second-year integrated curricula. This instrument was used to investigate whether there is a change in students' self-regulated learning skills over time. The SRL-SRS scores were compared using unpaired two-sample t-test. The relationship between SRL-SRS scores and academic performance was analyzed using regression analysis.

Results We have collected the first two datasets at the beginning and end of first-year integrated curricula. 76 out of 190 students have completed the first survey and 44 have completed the second survey. Six subscales (planning, self-monitoring, evaluation, reflection, effort, self-efficacy) measured by SRL-SRS instrument were analyzed. Results showed that students scored significantly higher $(P<0.05)$ on self-monitoring subscale when comparing the beginning and the end of the first year integrated curricula. Regression analysis revealed no correlation between each subscale of self-regulated learning skills and academic performance. Conclusions This study shows that the Tulane first-year pre-clinical integrated curricula does improve one subscale of students' selfregulated learning skills, self-monitoring. This would possibly be explained by the newly implemented self-directed learning teaching modality which emphasizes the LCME element 6.3 self-directed and life-long learning.

\section{INSTRUCTIONAL METHODS}

Poster: 328

Value of Complementary Review Material (ScholarRxTM Bricks) in Enhancing Preclinical Medical Student Learning and Performance

Gabi N. Waite, John L. Szarek, Carmine Cerra, Justin Collins, Pamela Lucchesi

${ }^{1}$ Geisinger Commonwealth School of Medicine, Scranton, USA

Purpose Medical school educators are challenged with simultaneously preparing students for patient care in their clinical years and for USMLE Step-1. Bricks (ScholarRxTM) are Step-1-level content items consisting of narrative text, images, mnemonics, and self-assessment items. Our purpose is to evaluate the use of the Bricks in achieving the dual goals of having students prepared for the clinic and being successful on Step-1. 
MethodsWe mapped complementary Bricks content to content in our hematology, cardiology, and pulmonology course blocks. The Bricks are available to MD2 students as non-mandatory exercises on the course portal. In addition to feedback on the usefulness of the Bricks from the class at-large, an incentivized cohort of students will be asked to keep a log-book of use and participate in a focus group at the end of the academic year. Students' academic performance in their second year will be correlated with their performance in their MD1 year and Step-1 scores. We also will interview stakeholders including course educators, and staff of Faculty Affairs and of the Office of Curriculum and Assessment.

Results Data collection and analysis using a mixed methods approach will be completed in May in time for presentation at IAMSE2019. Quantitative data will be the frequency of use of the Bricks and the academic performances of Brick users and non-users over two years. Feedback from surveys, focus groups and interviews will be analyzed using an inductive approach to identify initial codes, sub-categories, and themes.

Conclusion We anticipate that our study allows us to assess our hypothesis that the integration of complementary high-yield review material, in the form of Bricks, into our curriculum will help students balance the challenge of Step-1 preparation with the goal of readying themselves for patient care in the clinical years.

\section{INSTRUCTIONAL METHODS}

Poster: 329

Try This with Everyone: q Pilot Course to Improve Interviewing Skills In- and Outside Of Clinical Settings

\section{Anne Zinski \\ ${ }^{1}$ School of Medicine, University of Alabama at Birmingham, Birmingham, USA}

Purpose Initiating and documenting clear, purposeful encounters in healthcare settings has important implications for patient satisfaction, workplace dynamics, and reducing medical error. However very little research in medical education has explored learners comfort, skill, and aptitude in interpersonal skills outside of clinical settings. Therefore, our goal was to design an elective, small-group format course to foster improvement in interpersonal communication with an emphasis on refining interview skills, with the assumption that planning, data gathering, and summarizing could be practiced outside of clinic.

Methods The course included exploration of communication theory, selfassessment of skills across settings, didactic lessons, and scheduled homework. Learners further honed these skills via conducting and presenting findings from a series of service learning encounters. Each learner completed a self-assessment of perceived skills mastery consisting of 16 distinct interviewer behaviors on a 7-point Likert scale. Pre- and Postcourse ratings of pertinent skills, including information gathering, body positioning, and summarizing others messages, among others, were compared using paired $t$ tests.

Results Ratings from learners who had completed a pre- and post-course assessment were included in the analysis $(n=41), 95 \%$ response rate. At post-course, the largest improvements in mean skills ratings were in utilizing open-ended questions, using probing questions strategically, avoiding interrupting others, and reflecting others ideas to check for understanding. Conclusions In a course pilot that combined didactics, self-assessment, and strategic practice outside of clinic, medical students were able to improve effective interviewing behaviors, showing promise for strategic skills development outside of academic settings.

\section{INSTRUCTIONAL METHODS}

Poster: 330
Student Assessment of an Observational Experience of an Interprofessional Approach to Managing Medical Care for End-ofLife Cases

Jennifer Montemayor ${ }^{1}$, Christopher Unrein ${ }^{2}$

${ }^{1}$ Rocky Vista University College of Osteopathic Medicine Parker, USA

${ }^{2}$ The Denver Hospice, Denver, USA

Purpose Medical students must understand interprofessional roles, responsibilities and contributions given the impact of effective teamwork on patient outcomes. Some schools experience challenges offering meaningful interprofessional opportunities for pre-clinical students.

Methods Second-year students observed end-of-life case discussions through an interprofessional approach to managing patient care (including: bereavement counselor, chaplain, nurse, social worker, physician, volunteer, interdisciplinary group facilitator, music therapist), participated in a small-group debrief, and took an anonymous survey adapted from the Center for Health Science Interprofessional Education, Research, and Practice Post Assessment: Interprofessional Team Simulation Training to assess what was gained through the experience. IRB approval was obtained. Descriptive statistics were used to summarize data.

Results Of 158 responses, $73 \%$ agreed/strongly agreed it was a valuable educational experience. $83 \%$ agreed/strongly agreed it strengthened their appreciation for the physician leadership role. Seventy-five percent reported often/frequently observing leaders facilitating team function, creating information-sharing opportunities, discussing patient plans, and team members anticipating others' needs, consulting others, using communication skills that decrease error risk, and asking questions.

Conclusions These results suggest an observational experience was a valuable educational opportunity to learn interprofessional team dynamics and member contributions to patient-care case-management discussions and observe their future role modeled as well as the roles of other professionals. Therefore, a required, structured observational experience allowed student exposure to interprofessional team dynamics and important team functions such as leadership, information sharing, requesting assistance, and communication skills. Other medical schools may find that student observation of an interprofessional approach to patient case management discussions can be a valuable addition to the interprofessional curriculum as a means of enhancing student understanding of the importance of medical team function, particularly for programs which lack an affiliated teaching hospital or other health professions programs.

\section{INSTRUCTIONAL METHODS}

Poster: 331

Incorporating Home Visits into a Community Health Course to Reinforce the Importance of Social Determinants of Health

Amy Baldwin ${ }^{1}$, Achilia Morrow ${ }^{1}$, Laurel Murrow ${ }^{2}$, Suzanne Lester $^{1}$

${ }^{1}$ AU/UGA Medical Partnership, Athens, USA

${ }^{2}$ Mercy Health Center, Athens, USA

Purpose Social determinants play a major role in health. Although medical schools have introduced this concept into their curricula, it is unclear whether students connect this coursework to patient care. A recent revision of our community health course has given us the opportunity to directly reinforce to our students the relationship between social determinants and clinical practice by introducing home visits. These early experiences will allow students to recognize and incorporate these factors into patient care throughout their careers.

Methods A group of 8-9 first year medical students and 2 faculty preceptors (one clinical, one non-clinical) work with one of our community partners, Mercy Health Center, which serves uninsured patients in our area. During 
home visits for selected patients, our students take histories which include social determinants of health. With guidance from their preceptors, they work to develop a plan and add notes about the encounter to patients' charts. Templates and prompts are provided to help students gather information. Finally, students complete reflective writing and present exemplar patients to the entire class in a workshop format. Surveys will be used to formally gauge changes in student perspectives.

Results Student learning opportunities have included topics of migraine treatment, depression, specialist referrals, fragmentation of care, navigating the health care system and treatment of various chronic diseases. Additionally, promotion of a team approach has led to the investigation of legal assistance, transportation solutions, and assistance from community agencies. Workshops and reflections have revealed that students have identified factors beyond patient control and have recognized the role of family and social support, while broadening their view of the uninsured.

Conclusions Home visits are proving to be an effective way to teach students about the social determinants of health in the context of patient care, while providing a framework for the use of interdisciplinary approaches.

\section{INSTRUCTIONAL METHODS}

\section{Poster: 332}

\section{Collaborative Development of Concept-Based Cases for Cognitive Integration}

Tracy Fulton ${ }^{1}$, Michael Dell ${ }^{2}$, Leslie Fall ${ }^{3}$, David Harris ${ }^{4}$, James Nixon ${ }^{5}$, Ann Poznanski ${ }^{6}$, Amy Wilson-Delfosse ${ }^{2}$

${ }^{1}$ University of California, San Francisco School of Medicine, San Francisco, USA

${ }^{2}$ Case Western Reserve University School of Medicine, Cleveland, USA

${ }^{3}$ Aquifer and Geisel School of Medicine at Dartmouth, Hanover, USA

${ }^{4}$ University of Central Florida College of Medicine, Orlando, USA

${ }^{5}$ University of Minnesota Medical School, Minneapolis, USA

${ }^{6}$ California Northstate University College of Medicine, Elk Grove, USA

\section{Award Nominee}

Purpose The Aquifer Sciences curriculum is a resource for the development of tools to support learners' cognitive integration. We have recruited teams from six medical schools to develop virtual patient cases, each focused on the assessment of a single Integrated Learning Objective (ILO), and thus a single clinical decision, from the curriculum. These cases will provide clerkship students with deliberate practice in cognitively integrating, transferring and applying their basic science knowledge to safe and effective clinical decision-making.

Methods Each school recruited a team of basic science educators, clinical science educators, and senior students to collaboratively design cases. Teams were provided case authoring guides and webinar training on authoring clinical decision questions, basic science justification questions, and integrated explanations. Teams were then assigned a calibration case (i.e. provided the same concept, ILO and common condition) in order to define best practices and to ensure a consistent approach to authoring cases that draw on the concepts, teaching points, and Harm Statements of the ILO required to make the decision.

Results At this time, each school has completed a draft calibration case with questions and model explanations. Teams took different approaches to involve students, the number of answers for the clinical decision question, the length of the basic science justification, and the process for hand-off among and between team members. Most teams circulated drafts electronically but incorporated at least one team meeting. Results from the next phase of the project, in which each school will develop multiple cases to support either knowledge transfer or integration, will also be discussed.

Conclusions Curricula that support learners' cognitive integration are challenging to design. The case development framework described here requires learners to demonstrate an understanding of a basic science concept in making a provisional decision, leading to an entrustment action.

\section{INSTRUCTIONAL METHODS}

Poster: 333

Do You See What I See?: Using Art to Introduce Observation and Perspective in Large Group Human Structure Course Setting

\author{
Lauren W. Mazzurco ${ }^{1}$, Cody W. Long ${ }^{2}$ \\ ${ }^{1}$ Eastern Virginia Medical School, Norfolk, USA \\ ${ }^{2}$ Chrysler Museum, Norfolk, USA
}

Purpose Many schools are using art to build medical students skills of observation and diagnosis. Experiences are typically offered in museums and/or small groups. Using a large group format we introduced art to develop skills of observation, perspective, bias and communication in first year medical students. This abstract describes the project and outcomes.

Methods The session was attended by all first-year medical students $(n=$ 150) on their first day of class. As an introduction to their semester-long experience in the Human Structure Course with their anatomical donor and co-lead by a physician - educator and local museum educator, the session lasted 1.5 hours. Using a think-pair-share format, students made and discussed observations and variances in perspectives regarding an initial photograph. The museum educator then taught a systematic approach using a look, describe, think and connect framework with two different works of art. Using the think-pair-share format, students discussed and reflected on their personal perspective. Individually, they were asked to describe in writing how they thought this activity would impact their observational, collaboration and communication skills in their anatomical donor dissection small groups.

Results Art-related experiences in the undergraduate medical curriculum are most commonly held in the museum or small group setting. This was not a feasible option for us and thus, our session was designed in a large group, auditorium style lecture hall. Feedback from students and faculty was overwhelmingly positive. Students most commonly noted heightened awareness of needing to consider others perspectives as well as their own bias/connection in their observational interpretation.

Conclusions Despite the large group, auditorium style setting, using a think-pair-share format, led by a physician-educator and museum educator afforded our first-year medical students an opportunity to engage with using art to explore their own observational skills, perspective, bias and communication skills prior to their anatomical donor introduction.

\section{INSTRUCTIONAL METHODS}

Poster: 334

Factors Constraining Residents in Breaking Bad News at Moi University School of Medicine, Eldoret Kenya

David K. Chumba ${ }^{1 *}$, Irene Marete ${ }^{2 *}$, John Koskey Chang'ach ${ }^{3 *}$, Laban Ayiro $^{3 *}$

${ }^{1}$ Department of Medical EducationMoi University, Eldoret, Kenya ${ }^{2}$ Department of Paediatrics and Child Health, Moi University, Eldoret, Kenya

${ }^{3}$ Educational Foundations Department School of Education, Moi University, Eldoret, Kenya

Purpose Breaking bad news (BBN) to patients is one of the most common and often difficult responsibilities in the practice of medicine. The study sort to identify factors that constraints residents while performing this 
important task in the African context and to assess the effectiveness of training intervention using SPIKES protocol.

Methods A quantitative research, quasi experimental approach, was utilized where a questionnaires were used to collect the data. A purposeful sample of 80 physicians who are residents and provide treatment for patients with life threatening diseases in MTRH were selected for the study. The physicians were randomly assigned into two groups; experimental and control groups. Both groups filled a questionnaire then the experimental group were trained on delivering bad news to patients diagnosed with cancer.

Results A total of 80 residents the school of medicine were recruited. There were more men than women male $44(55 \%)$ and female 36 $(45 \%) .100 \%$ of the residents break bad news to patients more than 5 times in a month. Among the skills, the most totally grueling was how to be honest and not take away hope (20)25\% and dealing with patients emotions (29) $36.3 \%$. The perception of constraints did not change significantly after training when group A and B were compared. Time was not found to be a constraint as in other studies.

Conclusion Training residents in form a workshop, is not sufficient in difficult communication like breaking bad news. The findings in this study suggest that residents role in patient care may not be very clearly defined especially in breaking bad news tasks.

\section{INSTRUCTIONAL METHODS}

Poster: 335

Flipping or Flopping? Comparison of Student Performance and Perception of the Flipped Classroom for the Two-Campus Model

\section{Jennifer Montemayor}

${ }^{1}$ Rocky Vista University College of Osteopathic Medicine, Parker, USA

Purpose Commonly, medical schools additional-location campuses are required to have ? $50 \%$ of didactics originating on-site. Lectures are typically delivered bi-directionally via live-video technology to ensure equivalency. Flipped classrooms (FLIP) offer student-centered, activelearning opportunities. We asked if FLIP results in equivalent performance and perception between campuses, while enhancing curriculum origination per campus.

Methods Year 1 cardiovascular-physiology assessment and content, delivered via 7 FLIP sessions (pre-recorded lecture, self-assessment quiz, required small-group application session), was identical between campuses. FLIP facilitator:student ratio was similar. Homoscedastic t-tests analyzed FLIP exam-question $(n=52)$ and overall Year- 1 exam $(n=$ 20) performance between campuses. Post-course survey responses were analyzed by chi-Square to determine if differences in student FLIP perception existed between campuses.

Results The difference in FLIP exam-question performance between campuses was not significant $(2.21 \%, p=0.42)$. However, overall Year 1 exam performance was lower for UT ( $p=0.027)$. A significant difference was detected between Colorado (CO, $n=56)$ and Utah (UT, $n=53$ ) student responses for 2 of 11 survey questions. A greater proportion of $\mathrm{CO}$ vs. UT strongly agreed they enjoyed FLIP $(p=.042)$, and it was beneficial to learning $(p=.028)$; whereas, a greater proportion of UT vs. CO strongly disagreed. No significant difference existed in responses for the other questions. The majority of CO and UT indicated these FLIP benefits: (1) collaboration opportunity with peers and faculty, (2) peercollaboration on practice questions, (3) ease having questions answered vs. traditional lecture, (4) learning enhanced by pre-recorded videos and customized viewing experience. CO and UT ranked the top FLIP resources, also desired for future courses, as (1) application questions, (2) pre-recorded lectures, (3) self-assessment quizzes.

Conclusion With similar, possibly somewhat improved, performance outcomes compared with traditional-lecture format across two campuses,
FLIP offers an equivalent, collaborative, active-learning curriculum which supports institutional requirements of at least equal content originating from both campuses.

\section{INSTRUCTIONAL METHODS}

Poster: 337

\section{Flipping with Jeopardy: Problem-solving from Recall to Analysis}

Eve Gallman

${ }^{1}$ AU/UGA Medical Partnership, Athens, USA

Purpose I continue to seek ways to utilize active learning techniques in the classroom and to help my students develop enhanced critical thinking skills. Pre-class preparation coupled with in-class problem solving gives me an opportunity help my students learn how to apply information. Here, I describe my recent experience transitioning to a Jeopardy format for in-class problem solving.

Methods Pre-class preparation: Students were provided podcasts to be completed before class. Students were also provided written guidance to help them to fully appreciate the skill level they were expected to achieve before class time. On-line practice questions were available to help them assess their level of preparation. In class activity: Questions were presented using a Jeopardy style template consisting of a table of cells with 5 columns and 5 rows. Each cell links to a hidden question. Questions in a given column explored one topic, identified in a column header. Cells were color-coded to indicate whether the question (a) was primarily recall; (b) required application of information; or (c) required a higher level of analysis.

Results The arrangement of problems by topic and by degree of difficulty allowed students control over the pace and direction of the class time while ensuring that major target concepts would be encountered. Because the class got to pick the order of topics, they remained very engaged. Clear labeling of the types of questions available allowed students to build from simple to more complex concepts as they moved from recall to analysis.

Conclusion This format lent itself well to team discussion and goodspirited competition and helped the students to maintain interest. Importantly, it helped students learn to differentiate between fact knowledge and analysis.

\section{INSTRUCTIONAL METHODS}

Poster: 339

\section{A Simple, Effective Way to Demonstrate the Clinical Importance of the Perineal Body}

Bruce W. Newton

${ }^{1}$ Campbell University School of Osteopathic Medicine, Lillington, NC, 27546, USA

Purpose The perineal body is where three sets of muscles insert: the external anal sphincter, superficial transverse perineal, and bulbospongiosus $\mathrm{mm}$. During cadaveric dissection, it is a nondescript point between the vagina or penile bulb and anus. Students have difficulty identifying its location in cadavers. Clinically, it provides support for the pelvic organs. If an episiotomy is needed, lateral incisions are preferred to avoid cutting the perineal body which increases the possibility of pelvic organ prolapse.

Methods The demonstration, which can take place in the gross anatomy laboratory or lecture hall, consists of three students and a faculty member. Each of the four persons assumes the cardinal compass positions and extends a single hand toward the opposing compass point. The east and 
west persons represent the superficial transverse perineal $\mathrm{mm}$.; the north point represents the bulbospongiosus $\mathrm{m}$., the south point is the external anal sphincter. Standing fairly close together, the east and west points lock hands, then the north point grasps the top of those hands, while the south point grasps the other three hands from the bottom. You have now made a perineal body. Then ask each person to pull in their respective direction, this puts tension on the perineal body, while the faculty member, who is holding the bottom of the other three hands, lifts up slightly to further enhance the concept the perineal body provides pelvic organ support. Results/conclusions During the demonstration, the faculty member discusses how to form the perineal body and its clinical importance, the students now understand its formation and relevance. The high yield demonstration takes less than two minutes. After performing this demonstration in the gross lab for several sets of students, you can see them performing the demonstration to teach others.

\section{STUDENT SUPPORT}

Poster: 400

\section{Assessing the Mental Health Well-Being amongst Lake Erie College of Osteopathic Students}

Jennifer Allen, Molly A Johannessen, Melanie Dunbar

${ }^{1}$ Lake Erie College of Osteopathic Medicine, Erie, USA

Purpose To assess the mental health well-being amongst Lake Erie College of Osteopathic Medicine (LECOM) students in programs of Medicine, Pharmacy, Dentistry, and Graduate work across all campuses. Methods A cross-sectional web-based survey conducted in October 2018 among all students enrolled at LECOM $(N=247)$. Seven measures were utilized to assess the mental health status and stigma of mental health illness of LECOM students: Patient Health Questionnaire (PHQ-9) Depression Questionnaire, GAD-7 Questionnaire, Suicide Behaviors Questionnaire-Revised (SBQ-R), SCOFF Questionnaire, CAGE-AID Questionnaire, Life Event Checklist, and a modified version of the Community Attitudes Towards the Mentally Ill (CAMI) questionnaire.

Results and Conclusion The literature paints a grim picture of the status of mental health amongst medical students and physicians. Using the seven measures, the survey data was compared amongst and between various demographic identifications, educational programs, and various educational levels to identify any correlations between these factors and anxiety, depression, suicidality, eating disorders, drug and alcohol abuse, or stigma towards the mentally ill. Various comparisons revealed differences between programs and across educational level within programs as well as difference amongst demographics. Although the data observed through this survey is only a single snapshot of the mental health and well-being at LECOM, it is the first of this type to compare and contrast the mental health and wellbeing within one institution at various locations and across various areas of study all related to health care. The improvement of mental health within healthcare is imperative for the future of patients health and this study takes a great step towards gathering data towards this effort.

\section{STUDENT SUPPORT}

Poster: 401

\section{MS4s Teach \& Improve UCSF Bridges Year-1-Summer Academic Support Program}

Marieke Kruidering $^{1}$, Laila Fozouni ${ }^{2}$, Evan Whitehead ${ }^{2}$, Jacquelyn Withers $^{2}$

${ }^{1}$ Department of Cellular and Molecular Pharmacology, University of California, San Francisco CA 94143 USA
${ }^{2}$ University of California, San Francisco, USA

Purpose Medical students, particularly early learners, can experience challenges with academic performance. Many institutions have created robust, validated programs to support learners. Our recent curricular revision required redesigning our support program to incorporate several new elements, including the use of open-ended questions as assessments, and the move of USMLE Step 1 to after core-clerkships. Here, we describe a revision of the inaugural summer program incorporating support in this context.

Methods First-year medical students $(n=152)$ from the second cohort of UCSFs Bridges Curriculum were invited to apply, via messaging and targeted discussion with students coaches. Applicants were chosen based on academic or personal need. MS4s reviewed the 2017 summer program and implemented revisions in 2018. The 8-week summer program included (A) MS4-led small groups, (B) individual faculty mentoring, (C) resources, (D) CBSE-exit exam. (A) Small groups: MS1 \& MS4 presentations on year-1 material Discussion of MS4 selected-Qs from USMLE Step 1 Q-bank. (B) Faculty mentor meetings: Review Learning Plan. (C) Summer stipend \&Q- bank subscription.

Results Seven MS1s enrolled, all recommend program to peers CBSE scores trended up program well received overall $(>4 / 5)$ : Great way to synthesize first year topics with thoughtful and experienced MS4s and clinicians. It is a good blend of first-year medical knowledge, STEP-1oriented learning, clinically relevant focus, and advice about the aforementioned categories. Specific items evaluated > 4 Peer \& MS4 presentations. Guided Question bank use in group MS4s valuable peer instructors. Lessons learned MS1 rated review of year-1 materials (i.e. small groups and/or checkpoint questions) as not useful and preferred Peer \& MS4 presentations on preassigned year-1 topics. Q-bank overwhelming for MS1s without MS4 guidance.

Conclusions MS4 are superb near peer teachers Students prefer creating new presentations instead of passive review U-World questions can be used effectively for early remediation.

\section{STUDENT SUPPORT}

Poster: 402

Easing the Transition: Facilitating the Development of Effective Studying Strategies within the Medical School Curriculum

Taylor Barber, Stephen DePaul, Ryan Schwartz, Gregory Skedros, Kathleen Ackert, Michael McGuinness, Marina D’Angelo

Purpose Many students matriculate into medical school without having prior exposure to courses such as anatomy, histology, or embryology, which often serve as the foundation of the entire curriculum. Not properly preparing for these courses can lead to high levels of undue stress and poor academic performance. An optional two week pre-matriculation course designed to expose students to the content and difficulty of these topics was implemented to address this issue. Its success will be measured in terms of the effectiveness of various studying strategies that enrolled students developed as a result.

Methods The pre-matriculation course, Teaching Introductory Study Skills Utilizing Experience (TISSUE), debuted in the summer of 2017. Four rising second-year medical students facilitate the course and introduce content that students typically struggle with, while helping the incoming class experiment with different studying strategies in a low pressure environment. Three google surveys were administered to the Class of 2020 throughout their first term to gauge the perceived effectiveness of studying strategies that students were developing as the term progressed. Results Surveys were administered to the Class of 2020 during the first trimester of Structural Principles of Osteopathic Medicine (SPOM), which includes anatomy, histology, and embryology. Data analysis is currently underway. 
Conclusions We hope to find that the TISSUE course helps incoming students develop effective studying strategies earlier on in the term. The literature suggests that more efficacious studying techniques can improve academic performance and therefore lower levels of stress in the student population. This may also translate to better COMLEX and USMLE exam scores. Additionally, in allowing a select group of second year students facilitate the course, it opens up the line for communication, peer mentorship, and provides a support network to guide students through the often challenging transition into their first year of medical school.

\section{STUDENT SUPPORT}

Poster: 403

\section{A Wellness Blueprint for a Wellness Program}

Vivian Stevens, Sarah Hall, Jesse Chaffin, Kelly Dunn, Alicia Ford, Rachel Hardy

${ }^{1}$ Oklahoma State University Center for Health Sciences, Tulsa, Oklahoma 74107, USA

Purpose Alarming rates of burnout, suicide, and mental health concerns within the medical profession have ushered in an urgent call to address well-being strategies during medical training. While several excellent models for wellness programs have been described, the medical education community lacks a standardized approach for bringing this information to its medical trainees. As such, medical schools and residency programs are tasked with developing curriculum, guided by the literature and accreditation requirements. Additionally, wellness efforts may be siloed within institutions, diluting the robustness of a wellness culture on campus. This presentation will discuss the evolution of a wellness program being tailored to four years of medical school and the residency years.

Methods A plan for rolling out an integrated wellness program will be presented. Emphasis is placed on the initial convening of the Wellness Committee, steps towards integrating wellness offerings, and creation of new programming. Assessment measures, program content, and institutional collaboration will be discussed.

Results Within two years, this effort among students, faculty, and staff has led to a palpable campus-wide program. Focused committees, strong unit infrastructures, available adjunctive services, didactic/extracurricular programming, and various independent efforts anecdotally have spawned rapid growth of the wellness message on campus. A description of current efforts to solidify these gains will be presented.

Conclusion We attribute the success of the program to the support of leadership, campus-wide collaboration, high student involvement, and faculty/staff expertise. While wellness offerings existed prior to this effort, partnering with these programs and creating a wellness blueprint is contributing to a unified presence on campus. We believe this approach may serve as a useful model for institutions in the early-to-mid phases of wellness program development.

\section{STUDENT SUPPORT}

Poster: 404

Using Improv as a Tool for Fostering Mentorship Among Medical and Pre-medical Students

\section{Kathleen Herring}

\section{${ }^{1}$ AU/UGA Medical Partnership, Athens, USA}

Purpose Advising for pre-medical students can be reinforced and strengthened by involving current medical students. Medical students can improve their own communication skills and give back to medical community by mentoring students interested in healthcare. We focus here on the development of a structured series of activities, based on improv theater techniques to facilitate this relationship. A strong medical community depends upon open, safe relationships between colleagues; these workshops aim to create a foundation for this space and an area where students feel comfortable interacting and asking about current practices in medical education.

Methods Both medical and pre-medical students participate together in a series of integrated improv workshops focused on communication skills using curriculum developed in a previous study with medicals students and faculty at the medical school. The workshops take place over multiple, facilitated, 2-hour sessions, and students build communication and deep listening skills over time by participating in increasingly complex exercises. Focus groups with workshop participants will be conducted to gauge the viability of improv as a medium for mentorship.

Results Focus groups will be conducted with both pre-medical and medical students and feedback will be collected at the end of the sessions. It is anticipated that these results will lead to further refinement of the improv workshops with a special emphasis on fostering a sense of community and identifying methods of reinforcing communication between groups. Conclusion Mentorship between medical and pre-medical students is an important component of medical education. Improv theater is a useful tool for creating mentorship opportunities while also teaching the communication skills that are crucial for a successful career in medicine.

\section{STUDENT SUPPORT}

Poster: 405

Developing an Outcomes-Based Student Association to Provide Educational Programs for the Hispanic Community

Claudio Cortes, A. Celeste Farr, Virginia E. Uhley, Alyssa Perozich, Cheyenna Espinoza, Mallory Peterson, Belinda Asare, Connor Whitaker

${ }^{1}$ Oakland University William Beaumont School of Medicine, Rochester, USA

Purpose There is a need in medical schools to establish outcome-based student associations. Here, we describe the process of how the Latino Medical Student Association (LMSA) has been organized and created faculty and student-driven programs in partnership with the Catholic Charities of Southeast Michigan to promote learning for both the community and medical students.

Methods Program evaluation consisted of assessments to determine the effectiveness of learning through health educational activities, surveys using Behavioral and Emotional Rating Scale-2 to measure participants' behavioral and social skills, and to determine changes in communication skills with children, understanding of their needs, mentoring skills, and intercultural development.

Results LMSA has developed into an outcome-based student association benefiting both medical students and the surrounding community. Eightyseven M1-M2 medical students participated in two programs, mentoring and anatomy summer, where they mentored/tutored 120 children for 2,900 service hours. Medical students participated in 25 health-related educational activities, 9 field trips, weekly phone calls and direct interaction with mentees. Results of surveys in medical students $(n=42)$ showed high levels of student satisfaction $(M=5.13$, STD $=0.69)$, increased communication skills $(M=5.1, \mathrm{SD}=0.69)$ and understanding of the needs of Hispanic children $(M=4.87, \mathrm{SD}=0.65)$, high likelihood of recommending the program to other medical students $(M=5.48, \mathrm{SD}=0.74)$, and improved proficiency in mentoring(From $M=2.69, \mathrm{SD}=0.46$ to $M=3.47, \mathrm{SD}=0.5, z=$ 5.26, $p<0.001, r=0.574$ ). Of those surveyed, $83 \%$ stated they had learned new health information during the program. Parents felt their children improved in interpersonal skills, family involvement, intrapersonal strength, school functioning, and affective strength. Children's scores on pre/posttests increased significantly in 6 out 8 sessions. 
Conclusions We have established an outcome-based student association that successfully partnered with the Hispanic community. Both mentors and mentees have benefited in their interactions with each other, which include but are not limited to improvements in confidence, communication skills, and possible cultural humility.

\section{STUDENT SUPPORT}

Poster: 406

Do Student-Led Study Groups Improve Academic Performance and Self-Efficacy in Medical Students? A Prospective Study

Ahmed Hamed, Umair Syed, Joseph Mort, Selina Noramly, Deborah Barry

${ }^{1}$ University of Virginia, Charlottesville, Virginia 22903 USA

Purpose Cooperative learning has been shown to increase achievement and wellbeing in learners at a variety of levels. Medical students who form study groups tend to benefit from the social and academic support that these groups offer. However, there exists a paucity of research on cooperative learning and student-led study groups in medical education. The current research focuses on how best to create peer-led study groups, the impact on academic achievement, student self-efficacy, and student well-being.

Methods Medical students are invited to complete a questionnaire to participate in the study group program, and assigned to a study group. Students are assigned to a group based on their intake questionnaire which includes questions related to student demographics, study habits, goals for participation, self-reported academic performance, self-efficacy, and student wellbeing. Participants are asked to complete follow up surveys every four months, and results will be analyzed using appropriate statistical techniques.

Results This completely student-led initiative is in its final stages of development. Project leaders have completed the survey development and are currently completing the recruitment process. Based on preliminary recruitment, we expect 20 study groups to be formed including approximately 85 students. Conclusion This research will make a vital contribution to the literature related to both academic and social wellbeing of medical students. Finally, we expect that student-led study groups will encourage students to participate in a program designed and developed by their peers, and ultimately result in improved academic standing, reduced levels of stress, and greater perceived social integration amongst the participants, as well as be readily adaptable to other institutions.

\section{STUDENT SUPPORT}

Poster: 407

Implementation of a Novel Two-Week Practice of Medicine Course for Third Year Medical Students at the New York Institute Of Technology College Of Osteopathic Medicine (NYITCOM)

Rebecca Grohman ${ }^{1}$, Jason M. Golbin ${ }^{2}$, Barbara Capozzi ${ }^{3}$, Patrick M. OShaughnessy $^{2}$, Jerry Balentine ${ }^{1}$

${ }^{1}$ New York Institute of Technology College of Osteopathic Medicine, Glen Head, USA

${ }^{2}$ Catholic Health Services of Long Island, Rockville Centre, USA

${ }^{3}$ Touro College of Osteopathic Medicine, New York City, USA

Purpose A novel two-week course was designed to give third year medical students an introduction to the practice of medicine and to aid in the preparation for clerkships. The course was designed, in collaboration with Catholic Health Services of Long Island (CHSLI), to expose students to various topics not typically addressed within the traditional preclinical curriculum. As a secondary benefit, the course provided students with the opportunity for professional development through a flexible schedule to complete coursework on their own time.

Methods A two-week online course was designed at NYITCOM through a collaboration with CHSLI. An interdisciplinary team of providers including physicians, nurses, quality leaders, and administrators were enlisted to provide students with an overview of common topics in the current practice and business of medicine. During this course, students complete online technology-mediated learning assignments, reading assignments, and self-assessment. The course focuses on medical documentation, writing orders, communication skills, patient safety issues, and diagnostic errors. Students also learn the fundamentals of medicolegal issues, risk management, and aspects of medical billing and coding. The course recurs every two weeks throughout the academic year for new groups of students.

Results After more than $100 \mathrm{~h}$ of collaboration with CHSLI, the course was completed and is currently integrated in the third year curriculum for the class of 2020. Initial feedback from faculty and students has been positive. Additional analysis of student feedback regarding preparedness for approaching relevant topics during clerkships will be completed throughout the year.

Conclusion An innovative collaboration between NYITCOM and Catholic Health Services of Long Island provided a novel approach to teaching third year medical students about topics related to the practice of medicine.

\section{STUDENT SUPPORT}

Poster: 408

Evaluation and Comparison of Preclinical Medical Student Extracurricular Involvement and Subsequent Academic Success

\section{David Ramnaraign, Raza Sagarwala, Sara Barnett}

Purpose The formula for optimizing the probability of successfully matching to a top residency program has accrued an increasing emphasis on board exam performance (USMLE Step 1 in particular). Although there are many studies examining how Step 1 scores are affected by third-party study resources, practice exam scores, and studying habits, there are none that have examined how involvement in non-academic activities during the preclinical years can influence Step 1 scores. The primary purpose of this study is to therefore address the pervasive ideal that more time spent studying "at the sacrifice of volunteering, exercising, or research" will provide more long term benefit through higher board scores.

Methods The participants in this study are medical students at Saint Louis University School of Medicine who have recently taken Step 1 and voluntarily completed an online survey. The survey asked participants to report their average hours spent per week during their preclinical years on activities such as: volunteering, research, leadership, athletics, studying, and sleep. Further data on participants' gender, age, and Step 1 score were also collected.

Results Preliminary results from $43 \%$ of respondents $(n=76)$ reveal a significant difference in the average step scores of male and female respondents (241 vs. 226). Additionally, female students on average reported spending more hours per week volunteering ( 9.7 vs. 4.3 ), whereas male students prioritized studying (53 vs. 48 ), research (4.4 vs. 3.2 ), and athletics (7.7 vs. 6.4 ). No statistical difference is seen in time spent sleeping between genders ( 52.5 vs. 52.3 ).

Conclusion When stratified by gender, there seems to be a significant difference in how third year medical students spend their time during the preclinical years and score on USMLE Step 1 . These preliminary data suggest that current medical education curriculums may be suboptimal for female students who wish to spend more time engaged in their communities than their male counterparts. 


\section{STUDENT SUPPORT}

Poster: 409

\section{Dissatisfaction with Completion of a Medical Degree in Both DO and MD Programs}

\author{
Mark J. Hernandez \\ ${ }^{1}$ University of Central Florida College of Medicine, Orlando, USA
}

Purpose Around 1500 medical graduates responded this year in a survey that they would not pursue a career in medicine if they could revisit this career choice. This abstract aims to compare the aggregate data available from national graduation questionnaires for graduating seniors for the past 10 years to try to comprehend some of the reasons for this effect. Methods The feedback from the All Schools Summary Report of the Medical School Graduation Questionnaire, published by the American Association of Medical Colleges (AAMC), and Survey of Graduating Seniors Summary Report, published by American Association of Osteopathic Medical Colleges (AACOM), was analyzed for similarities and differences. The reports published between 2008 and 2018 were reviewed. These reports provide a snapshot of the students self-reported experiences and satisfactions with their medical education and are readily available online to the public.

Results Of the 19,537 MD graduates $83 \%$ completed the AAMC survey, and of the $6,350 \mathrm{DO}$ graduates $77 \%$ completed the AACOM survey. In $2018,7.9 \%$ of MD graduates who completed the survey would not, or probably not, have chosen to become a physician, and $4 \%$ of DO graduates wished they had pursued something else. In 2010, around $7.5 \%$ of MD graduates and $6 \%$ of DO graduates responded in the same manner. Conclusion The satisfaction score analysis for the past ten years (20082018) indicated a progressive improvement in the overall student satisfaction with the quality of medical education. It is unclear however of what factors led to the dissatisfaction with completion of the medical degree in a small, but significant, number of medical graduates during the past eight years.

\section{STUDENT SUPPORT}

Poster: 411

\section{Predictors of Retention in the Occupational Therapy Assistant Program Admissions Process}

\section{Leah N. Sowers}

${ }^{1}$ Jefferson College of Health SciencesRoanoke, USA

Background The field of occupational therapy (OT) is fast-growing and in high demand. The need for occupational therapy assistants (OTAs) is expected to grow $28 \%$ by 2026 , creating further demand for OTAs (U.S. Department of Labor, n.d.). Currently, there are only nine accredited OTA academic programs in the state of Virginia. As a result, the number of OTA program applicants far exceeds the number of available openings at Jefferson College of Health Sciences (Jefferson College) in Roanoke, Virginia. This places pressure on the OTA program to admit the best candidates who will complete the program in its two-year timeframe, thus securing retention rate and pass-rate statistics. In order to achieve this, in 2015 the OTA program at Jefferson College created and implemented a Selective Admissions Process (SAP) to screen and admit the most qualified candidates for the program. The purpose of this research is to identify criteria of applicants that predict successful competition of the OTA program. Implications of this research are manifold and include increased retention at Jefferson Colleges OTA program, an increase of employable OTAs in Southwest Virginia, and development of a model selective admissions process for similar OTA programs. The purpose of this study was to identify criteria within the selective admissions process that predict success in the OTA program.

Methods The study involved analysis of data for 90 students (45 pre- and 45 post-selective admissions process) admitted to Jefferson Colleges OTA program between 2013 and 2016. Areas identified for admissions screening included the following criteria: high school or previous college grade point average (GPA), previous healthcare exposure and experience, community service and volunteer work, letters of recommendation, honors, awards, recognition, resume, personal statement, special skills, sample works, admissions interview, and on-site writing sample. Criteria were scored by OTA program faculty according to rubrics. A total score, which included all criteria, was used to rank applicants within their cohort and offer admissions to the highest scoring applicants until the class was filled.

Results Scores on criteria identified for admissions screening and the total score were analyzed. The total score was determined to be a significant predictor $(p=.016)$ of program completion for the 45 students who went through the selective admissions process, accounting for approximately $26 \%$ of the variability in degree completion for those students.

Conclusion/Discussion The results of this study validate the current SAP process used by the OTA program at Jefferson College to select applicants. The results establish that criteria identified in the selective admissions process are significant predictors of completion of the OTA program.

\section{STUDENT SUPPORT}

Poster: 413

\section{What's My Role: Medical Student Risk and Suicide Prevention}

\section{Cynthia A. Standley, Rebecca E. Fisher}

Purpose The presence of suicidal ideation in healthcare training students is a source of concern. Depression or depression symptoms affect nearly $27 \%$ of medical students. Do you know what to do when you see a student in your classroom struggling? A court ruling in Massachusetts in 2018 found that colleges have an obligation in some cases to prevent suicides and that there are situations where professors could be sued for failing to do so. Importantly, suicide rates vary by age, gender, race, and ethnicity. Classroom environments are places where students may come across aggressive comments that are disparaging and may affect their mental health. While macroaggressions are blatant, microaggressions are often unconscious. A challenge for education practitioners is how to effectively address such microaggressions in the classroom.

Methods Through 3 interactive sessions with health professions students, faculty and staff, we captured participants responses to questions on campus culture and suicide risk. In this poster, we will provide an overview of medical student risks, including microaggressions that are more subtle and macroaggressions that are more blatant and how these can affect depression and suicide risk. We will provide a sense of what students may face in the classroom, vs what they may face in the clinical setting and then discuss what educators can do to mitigate some of these risk factors for students.

Results Our sessions have led to a more positive campus culture, increased the number of informed faculty and influenced a new formal curricular session on targets of bias.

Conclusion Suicide is the second leading cause of death among medical students (second to accidents), yet no medical organization is tracking these suicides. Our hope is for the non-clinician to gain confidence in becoming someone students can check in with, look up to, and talk openly with, giving them hope and reducing their stress.

\section{TBL PBL}

Poster: 500 
"Describe What You See": a Pilot Project in Converting ProblemBased Learning from Text to Videos in Preclinical Medical Student Psychiatry Education

\author{
Mary Steinmann, Sean Ferrell \\ ${ }^{1}$ University of Utah, Salt Lake City, USA
}

Award Nominee

Purpose Problem-Based Learning (PBL) is a commonly-utilized teaching method in preclinical medical student education. PBL consists of cases that students work through in small groups with a faculty facilitator, who may or may not be a content expert. At our institution, the cases are typically presented in text format with progressive disclosures as the case unfolds. However, important psychiatric concepts, including mental status examination findings, are difficult to learn. Video has been used widely in psychiatric teaching. In an effort to improve student engagement and understanding of observable psychiatric findings, we developed and piloted a series of progressive-disclosure cases that use videos of simulated psychiatric patient encounters to stimulate small group discussion and problem solving, facilitated by faculty who are not content experts. Methods Three progressive-disclosure video cases were developed to teach depression, psychosis, and peripartum mood disorders. The videos employed psychiatric residents as actors to reduce cost and enhance authenticity. The cases were piloted during weekly 1-hour PBL sessions in the second-year medical student Brain and Behavior course. A separate facilitation guide was developed describing key teaching points of each case disclosure, and offering suggestions to help faculty who were not experts in behavioral health facilitate student discussion. A 30-minute training session was held with faculty facilitators prior to each session to review the case and answer questions. The activity was assessed on standard end-of-course evaluations.

Results One hundred twenty second-year medical students participated in the pilot project. Each case took approximately 1 hour for groups to complete. Ninety-two percent of students on end-of-course evaluations reported that the videos used in PBL increased the authenticity of the cases. $91 \%$ of students reported that PBL sessions enhanced their learning of course content.

Conclusion Video-based PBL is an engaging method to teach psychiatric principles including mental status examination/observation, diagnosis, and treatment in preclinical psychiatric education.

\section{ELEARNING}

Poster: 501

\section{Transformation of Didactic Medical Content to Interactive Video} Lectures (IVL)—Mass Production

\section{Alice Akunyili, Hany Ibrahim, Kanee Lerwill, Rodolfo Bonnin \\ ${ }^{1}$ Herbert Wertheim College of Medicine, Miami, USA}

Purpose Conversion of didactic lectures in the curriculum at Herbert Wertheim College of medicine into an innovative instructional method, Interactive Video Lectures (IVL), seeks to transform medical content into eLearning options that apply learning science and data science to improve access to medical education, fit remote audiences and allows flexibility of content delivery and adaptive learning, or learner controlled content sequence. Methods The first step was to identify the utility of IVL within the larger framework of our curriculum, and garner support from key stakeholders. Our team of physician educators had a mandate from the administration, so the challenge was to secure the support and the resources from faculty, staff, and other key stakeholders. The second step was to assess the feasibility of transforming complex didactic content and determining which parts of the curriculum could be converted efficiently. The final step was to design a quality assessment and improvement process using a standardized rubric incorporated into a production process that can be scaled.

Results To achieve our objective of transforming didactic lectures to IVLs, we created a process aimed at efficiently developing and converting large amounts of medical content. In essence, we created an assembly line for IVLs. It is a production process in which elements of the IVL are added as the semi-produced IVL moves from one station to another in a specific sequence, until the IVL is fully produced. Each station has functions specific to the elements being incorporated. Quality checkpoints are positioned at different stations throughout the assembly.

Conclusion Aligning institutional objectives with production capability and subject matter expertise is a major challenge of e-Learning transformation in Medical Education. Creating an IVL assembly line allows for efficiency and quality checkpoints, and can be scaled up or down according to the purpose, resources, priorities, timeline and quality requirements.

\section{TBL PBL}

Poster: 502

Team-Based Learning Scores Correlate with NBME Style Quizzes in Problem-Based Learning Curriculum

Jaya Yodh $^{1}$, Stephanie Ceman ${ }^{1}$, Kashif Ahmad ${ }^{1}$, Kaustubh Bhalerao ${ }^{2}$

${ }^{1}$ Carle Illinois College of Medicine, Champaign, USA

${ }^{2}$ University of Illinois at Urbana Champaign, Champaign, USA

Purpose Carle Illinois students start off their medical curriculum with a Foundational Elements course based on the NBME outline for Foundational Content. This course is meant to normalize and assess proficiency of students entering the program from many different backgrounds. The course also aimed to compare learning from Team-based Learning (TBL) and National Board of Medical Examiners (NBME) style quizzes.

Methods In a foundations course based on the NBME outline for Foundational Content, both TBL and weekly quizzes using NBME style questions were used to assess student understanding of content across genetics, biochemistry, immunology, physiology, and cell biology. IRB was obtained and students consented to participate in the study. The students participated in a weekly TBL, consisting of an individual readiness assessment test, group assessment and applications activity, based on pre-readings and lectures. The students also participated in weekly quizzes formed using NBME style questions matched by course objectives and keywords from question bank. At the end of the course students took a NBME subject exam based on Foundations content and 29 students consented for sub-scores to be released for analysis.

Results Scores for TBL and weekly quizzes were compared using a Pearson correlation by topic and by week. Correlations for topics ranged from 0.20 to 0.50 and correlation for weekly performance was between 0.2 and 0.4 but overall average performance on quizzes and IRATs was 0.68 .

Conclusion Well-written TBL IRATs can be indicative of student performance on high stakes exams in the same way that correlates with NBME style quiz indicators for student performance.

\section{ELEARNING}

Poster: 503

Student Physical Therapist Use of Digital Textbooks and Electronic Media

Debbie Ingram 
${ }^{1}$ University of Tennessee at Chattanooga, Chattanooga, USA

Purpose Digital textbooks have become an attractive option for students, faculty, and administrators (Daniel). Researchers have discovered several unique advantages to digital textbooks including features such as interactive learning modules, multimedia experiences, references, and resources for topics of interest (Keena). Staiger found that students enjoyed the search feature, as well as the ability to electronically highlight, bookmark, and annotate. Eno found that nursing students appreciated the lighter weight and significant reduction of paper. Rockinson et al. found that students who used digital textbooks for their courses had significantly higher perceived learning and psychomotor learning." Multiple authors have reported students prefer printed textbooks over digital. The purpose of our study was to identify the perceptions and use of digital textbooks and electronic media by doctoral physical therapist (DPT) students.

Methods A 30 item survey instrument was created by the authors and distributed electronically using Qualtrics. The survey addressed use and preference of traditional books vs. digital texts. Students from 2 DPT programs in different regions of the country completed the survey.

Results One hundred twenty-five DPT students completed the survey $(48 \%)$. The majority $(72 \%)$ were female. Most of the students owned devices capable of viewing digital texts (98\%). $7 \%$ had previously purchased a digital textbook and $78 \%$ preferred to purchase printed texts. Students preferred digital textbooks for portability (83\%), lower costs (72\%), and easiest means to search subject matter (57\%). Printed texts were thought to be easier to read (91\%), less tiring on the eyes $(95 \%)$, improved comprehension (86\%), easier to remember material $(88 \%)$, easier to use for exam preparation $(83 \%)$, and more suited to some individual learning styles $(86 \%)$.

Conclusions The findings from our study mirror the findings of previous studies. It is important for DPT programs to be familiar with innovative new educational strategies such as digital textbooks and electronic media.

\section{TBL PBL}

Poster: 504

\section{Implementation of Team-Based Learning (TBL) in a Second Year Medical School Course: Does Prior Experience with TBL Improve Impact of this Pedagogy?}

Gonzalo A. Carrasco ${ }^{1}$, Matthew Gentile ${ }^{1}$, Michelle L. Salvatore ${ }^{1}$, Osvaldo J. Lopez ${ }^{2}$, Kathryn C. Behling ${ }^{1}$

${ }^{1}$ Cooper Medical School of Rowan University, Camden, USA

${ }^{2}$ Hackensack Meridian School of Medicine at Seton Hall University, Nutley, USA

Purpose TBL has been used in several educational environments including medical education. At our institution, we have shown that use of TBL in a first-year Infectious Diseases (ID) course resulted in improved final examination performance and fewer course failures. Therefore, we decided to implement TBL in the second-year Womens Health (WH) course to improve acquisition of course content related to sexually transmitted infections (STI). Here we examine how prior experience with TBL in the ID course affected TBL performance, approximately 12 months later, in the WH course. Methods The ID course has weekly TBL exercises that cover all the course material, while the WH course has one TBL that only covers a small portion of the course material (STI). Final examination and TBL individual readiness assurance test (iRAT) scores in the ID and WH courses from three classes of students $(n=226)$ were obtained with Rowan institutional review board approval. Statistical analyses were performed using IBM SPSS Statistics (IBM Corp., Armonk, NY).

Results As we previously reported in the ID course, WH iRAT scores were significantly correlated with $\mathrm{WH}$ final examination performance $(r$ $=0.33, p<0.001)$. Additionally, individual student average iRAT scores in the ID course were highly correlated with ID $(r=0.45, p<0.001)$ and WH $(r=0.31, p<0.001)$ final examination scores, as well as with iRAT scores in the WH course $(r=0.35, p<0.001)$. Finally, WH iRAT scores were significantly higher than individual student average ID iRAT scores ( 9.19 vs. $7.40, p<0.0001$ ).

Conclusions We have previously reported that TBL-iRAT scores are predictive of performance on summative course examinations of medical knowledge. In this study, we found that TBL scores are also predictive of future performance in other courses. Interestingly, the current study also suggests that prior TBL experience likely contributes to better TBL performance, as measured by iRAT scores, in future courses.

\section{ELEARNING}

Poster: 505

\section{Spices Model in a Mini Course Development}

Jaehwa Choi, Hye Young Yoon, Robert McKallip

${ }^{1}$ Mercer University School of Medicine, Macon, USA

Purpose The SPICES model of educational strategies has been implemented for curriculum development by many institutions. It is a student-centered (versus teacher-centered), problem-based (versus information-gathering), integrated (versus discipline-based), communitybased (versus hospital-based), elective (versus uniform), and systematic (versus apprenticeship-based) approach. To help students learning in basic medical sciences, a student-centered and integrated summer cardiology course named Summer Cardio Camp was developed.

Methods In an organ-based basic medical science curriculum, cardiology is undoubtedly one of the most challenging topics. It is particularly challenging for those who have not had any foundational courses in physiology. Thus, the goal of Summer Cardio Camp was to guide students for self-directed preparatory learning in medical cardiology by providing asynchronous online mini-courses. First, an institutional subscription to a series of commercially available online lectures was made available to all students. Among over 100 hours of lectures in cardiovascular physiology, pharmacology, and pathology, a selected list of lectures that could fit in a 4-week self-studying program was prepared. Relevant readings of textbooks were provided to students as well for those who preferred to read than listen to the lectures.

Results This course was offered not as a mandatory but as a non-credit earning, voluntary mini-course. Weekly assignments were given to the students. Faculty gave immediate feedback on their assignments. Students' feedback from the course evaluation varied from enthusiastic to uninterested. Conclusions In conclusion, a student-centered and integrated mini pilot course "which was not mandatory but self-directed with guidance by a teacher" was developed to aid students in learning cardiology. To enhance the effectiveness of self-directed learning, the timing and duration of the mini-course will be re-designed based on students' feedback and their performance on the summative exam.

\section{TBL PBL}

Poster: 506

Achieving Content Integration in an Undergraduate Medical Education Curriculum Through Interprofessional Team-Based Learning

Amanda J. Chase, Daniel Griffin, Patricia C Rose

${ }^{1}$ Nova Southeastern University, Ft. Lauderdale, FL 33328 USA

Purpose Team-based learning (TBL) has emerged in medical curricula as an educational method that promotes learning of the foundational and 
health sciences. Our curriculum begins with a fundamentals course which includes active learning and TBL sessions facilitated by content experts who design content around their discipline. The fundamentals course is followed by integrated systems courses. For the systems courses, we developed TBL sessions that emphasize synthesis of integrated content spanning several disciplines. This methodology involves a process referred to as scaffolding, whereby students learn foundational concepts in isolation during the fundamentals course and then synthesize these concepts in combination with multidisciplinary learning objectives in subsequent courses.

Methods Our TBL process follows traditional best practices established by the TBL Collaborative. To transform TBL sessions in our integrated systems courses we modify the content and delivery of each session, using interprofessional teams to achieve integration and higher order learning. To add to this, content is presented and assessed using clinical scenarios that integrate multidisciplinary learning objectives.

Results We present approaches and challenges to the transformation of discipline-based instruction into integrated TBL experiences. In addition, we present outcomes of assessment on learning objectives between the fundamentals and systems courses. To assess the impact of redesigned TBL, we describe results of a survey to assess the students' perspective of engagement. Conclusions Our measured approach to introducing integrated TBL sessions exemplifies the scaffolding process by which learners develop stronger metacognitive skills when they are exposed to concepts in isolation prior to applying them in combination. Future directions include evaluating outcomes over several consecutive student cohorts.

\section{ELEARNING}

Poster: 507

C4tech: Virtual Connections Between the Classroom, Clinician, and Community Clinic to Bridge the Education/Practice Gap

Paige McDonald ${ }^{1}$, Howard Straker ${ }^{2}$, Jacqueline Barnett ${ }^{3}$, Gregory Weaver $^{1}$

${ }^{1}$ George Washington University School of Medicine and Health Sciences, Washington, D.C., USA

${ }^{2}$ Physican Assistant Program, George Washington University School of Medicine and Health Sciences, Washington, D.C., USA

${ }^{3}$ Physician Assistant Program, Duke University School of Medicine, Durham, USA

Purpose Learning Health Systems (LHS), proposed as a model to integrate evidence for continuous quality improvement and learning, require connections between the healthcare education and delivery systems. Faculty leveraged blended learning in a Physician Assistant (PA) course sequence to connect the classroom and clinic for active learning. Through online technologies, we connected students in the classroom with clinicians from clinics with diverse patient populations to develop quality improvement projects addressing current disparity concerns within the clinical setting (C4Tech). This pilot seeks to understand if C4Tech can bridge the classroom/clinic divide for authentic learning and practice improvement.

Methods Investigators adopted a case study approach to access the efficacy of the educational intervention. Multiple assignments-community/ clinic profiles, VoiceThread presentations, and final Performance Improvement Continuing Medical Education (PICME) projects-were analyzed for overall and highest level of learning using Bloom's Taxomony of learning domains. Final PICME projects were also analyzed for integration of course concepts, clinic knowledge, and evidence for practice improvement.

Results Community profiles exhibited wide variation in overall and highest level of learning, with 5 out of 8 teams achieving apply as the highest level. VoiceThread presentations exhibited less variation in highest and overall level of learning, with 6 of 8 achieving evaluate as the highest level. All 8 PICME projects achieved integration of course concepts, clinic, knowledge and evidence for practice.

Conclusion Application of the C4Tech model promotes high levels of learning and knowledge integration. It can also bridge the classroom and the clinic for active learning and quality improvement. Additional research is required to determine the factors that influence variation in the levels of learning achieve across clinic/student teams. Future applications of the model should consider the impact on quality metrics or patient outcomes as well as clinician learning.

TBL PBL

Poster: 508

\section{Evaluation of Premedical Module at Duke-NUS Medical School}

Lee Cheng Jie Irene, Sarada Harichand Bulchand

${ }^{1}$ Duke-NUS Medical School, Singapore City, Singapore

Award Nominee

Purpose Duke-NUS medical school, a graduate entry medical school, the first in South East Asia, adopts Team-based learning (TBL) in delivering preclinical content. To recruit prospective MD students, we developed a Premedical Module using TBL as the main pedagogy to drive critical thinking and communication skills. The module, runs for 16 weeks, and is offered to local undergraduate students across faculties. This abstract describes the project and its outcomes.

Methods The module has run for 7 years. A wide range of topics in biomedical science were delivered in TBL format together with team assignments and field trips. The course was facilitated by research fellows. Students who completed the module with outstanding performance were shortlisted as Premed Scholars. These individuals received clinical shadowing experience to gain insight into the roles of medical professionals in Duke-NUS. To evaluate the effectiveness of the Premedical module as a recruitment tool, we analyzed students' reaction to the module, using thematic analysis on session evaluation reports, and also determined the application and acceptance rate to the MD programme.

Results One hundred sixty-nine students took the module from 2010 to 2015. 91 students were selected as Premed Scholars. Common perceptions of the module included an enriching exposure to different aspects of cutting edge medical research, and a positive learning experience through TBL which gave an insight into the unique pedagogy used at Duke-NUS. On average, in each academic year, 24\% of Premedical modules takers applied, and among those who applied an average of $72 \%$ were accepted to the MD programme. Among the Premed Scholars, on average 50\% applied and $78 \%$ were accepted to the MD Programme.

Conclusion The Premed module provides an opportunity to attract high quality candidates. Experiencing the same teaching pedagogy of the MD programme, and routine engagement with the medical school facilitates quality applicants and entrants to the MD programme.

\section{TBL PBL}

Poster: 510

\section{Optimization of Small Group Learning}

Mark Best ${ }^{1}$, Anthony Pappas ${ }^{1}$, Mark Coty ${ }^{1}$, Swapan Nath ${ }^{2}$

${ }^{1}$ LECOM-Bradenton, Bradenton, USA

${ }^{2}$ TCU and UNTHSC School of Medicine, Fort Worth, USA

Purpose Currently medical education strategies and processes have incorporated or focused on active learning (including collaborative learning) 
for knowledge acquisition for practice, education program objectives (including teamwork, interpersonal and communication skills and professionalism), and outcome measures (including national board scores, formative assessments by educators). Continuous improvement of our ability to impact self-regulated learning, clinical reasoning, medical knowledge, professionalism, and teamwork skills is necessary. Improving educational quality/effectiveness, increasing educational efficiency, and increasing student and facilitator satisfaction are the goals of this synthesis. Methods This synthesis is based on neurobiology of learning, cognitive psychology theories (e.g. metacognition, transdisciplinary thinking, cognitive integration), process management methods, quality improvement techniques, and implementation science. We use Google Scholar, ERIC, and MEDLINE for our literature search. Inclusion criteria key words are: team-based learning, problem-based learning, case-based learning, casebased collaborative learning, cognitive load theory, integrating basic sciences and clinical content and experiences, learning environment, memory, encoding and retrieval, sensory processing, experiential learning, reward and reinforcement, implementation science. Exclusion criteria is non-English publications.

Results A critical synthesis of small-group instructional designs for active learning, including team-based learning (TBL), problem-based learning (PBL), case-based learning (CBL), \& case-based collaborative learning (CBCL), describes the structure, constraints, processes, and outcomes, and compares and contrasts these strategies. We simplify an optimal design and processes to increase cognitive knowledge and experiential knowledge in the learning environment.

Conclusion This review provides educators with a summary of what works well for small-group instructional designs, within the time constraints and other limited resources, to assist them in operationalizing the optimal students' educational experience for the needs of their patients, or community.

\section{TBL PBL}

Poster: 512

The Impact of a Team-Based Learning Exercise in the Understanding and Retention of Medical School Nutrition Education

\section{Surya Khadilkar, Lynn Cialdella-Kam}

${ }^{1}$ Case Western Reserve School of Medicine, Cleveland, OH, USA

Purpose Currently, limited curriculum time is dedicated to nutritional content and many medical students lack confidence in applying their nutritional knowledge to a clinical context. A team-based learning exercise (TBL) was implemented in the Case Western Reserve School of Medicine (CWRU SOM) curriculum during the preclinical nutrition, gastroenterology and biochemistry block to increase nutritional knowledge and confidence. We investigated if TBL is an effective modality to educate students on the fundamentals of nutrition and increase retention of this information.

Methods The TBL exercise was implemented into the required preclinical curriculum for first year medical students. In order to evaluate the efficacy of the team-based learning session, an optional posttest was administered both to students who received the intervention and to a randomly selected medical student cohort that did not participate in the educational intervention, and their results were compared.

Results The TBL has been implemented in for current the first year medical students. In 2018, 18 first year students and 14 second year students (total $n=31$, years 1-2) completed the posttest survey out of a possible 50 randomly selected students in each year level respectively. Based on student evaluations of the TBL faculty, students indicated that (1) have valued the TBL exercise as an educational modality and (2) enjoyed the input from the facilitators.
Conclusion A lack of basic understanding in nutritional counseling motivated us to seek out a new opportunity to incorporate more nutritional content into the curriculum. The intervention appears to have a positive influence on students confidence in nutritional knowledge. Further steps include interventions to test the retention or change in both knowledge and confidence.

\section{TECHNOLOGY \& INNOVATION}

Poster: 600

Breaking Frontiers of Basic Medical Sciences to Educate Students from Non-health Science-Related Programs

Juan Pablo Carrillo Vargas, Mildred Lopez Silvia Olivares, Belinda del Carmen Carrion-Chavarria

\section{${ }^{1}$ ITESMAve. Morones Prieto 3000, 64710 Monterrey, N.L., Mexico}

Purpose In order to promote multidisciplinary teamwork across different disciplines, it was designed the I Week. This full time immersion program includes challenging activities to develop generic competences on students. The aim of the study was to measure the satisfaction of the students registered on the Basic Medical Sciences I Week activities.

Methods The study had a mixed, transactional and descriptive design. Participants were 148 students from different programs, 68 from the School of Medicine and Health Sciences (EMCS from its acronym in Spanish) and 80 from non-health sciences programs. There were offered six activities, for example: Anatomy to all: a virtual focus for understanding the reality and Human anatomy, art, science and technology among others. There was applied a satisfaction survey (Cronbach Alpha 0.94) and interviews to students at the end of the week.

Results The response rate on the survey was $28 \%$. The average overall satisfaction from 42 students was 4.71 on the six activities on 1-5 scale. Interviews with 16 students from engineering, accounting, finance and art showed that $93.8 \%$ of them decided to join the activity voluntarily, and $87.3 \%$ would recommend their activity it for future classes. Collaboration among students from different disciplines changed their perception about teamwork on health science related topics.

Conclusion The faculty had the opportunity to challenge students from different programs on a multidisciplinary teamwork. Both health sciences and non-medical sciences students learned how to deliver a solution from the medical field and overcome some misconceptions around the selected topic.

\section{TECHNOLOGY \& INNOVATION}

Poster: 601

\section{Making the First Step a Virtual One}

Becca Gas, Katie Cornelius, Heather Billings

${ }^{1}$ Mayo Clinic College of Medicine and Science, Rochester, USA

Purpose We aimed to address the lack of interprofessional education (IPE) resources across five schools within the College of Medicine and Science at our institution. Multiple gap analyses have shown a need for explicit IPE training and activities for learners and faculty at our institution. With 4000+ faculty teaching and assessing, and 3500+ learners from post-baccalaureate to post-doctoral programs, the diversity of learning environments, pedagogies, outcomes, and expectations is complex. This largesse poses immense challenges and generates significant competing demands on a finite number of organizational resources including physical space and dedicated staff.

Methods To address the lack of IPE tools and resources at our institution, we created and launched a Virtual Center for IPE in conjunction with an enterprise-wide forum for educators. The IPE Virtual Center is a searchable 
website on our institutions intranet that houses a variety of tools and resources in a centralized location for students, trainees, faculty, physicians, and staff. It allows for robust collection of analytics to inform future programming, justify resource acquisition, and optimize opportunities for faculty development to serve as a driver for organizational change and innovation.

Results We have found this model to be successful at our institution to launch faculty development programming, IPE initiatives, and instructional design projects. By establishing a Virtual Center, we have allowed for necessary coordination, curation, dissemination and communication channels to drive awareness, desire, knowledge building, assessment and recognition in a timely, efficient, and sustainable manner.

Conclusions While an IPE Virtual Center does not require an immense amount of institutional resources, it is crucial for generating momentum to drive acquisition of additional resources. The virtual center model is both feasible and generalizable to other institutions hoping to implement a specific program or set of education resources justified by engagement and impact data.

\section{TECHNOLOGY \& INNOVATION}

Poster: 602

\section{The Effects of Two-Dimensional and Virtual Three-Dimensional Pelvis Anatomy on First-Year Medical Students Learning}

Edgar R. Meyer ${ }^{1}$, Amber M. James ${ }^{1}$, Kenneth Thompson ${ }^{2}$, Dongmei $\mathrm{Cui}^{1}$

${ }^{1}$ University of Mississippi Medical Center, Jackson, USA

${ }^{2}$ Millsaps College, Jackson, USA

Purpose Traditionally, the pelvis has been an anatomical region of great complexity. There is limited research on the efficacy of virtual pelvis models over traditional learning. The purpose of this study was to compare the impact of two-dimensional (2D) images of pelvic anatomy and a virtual three-dimensional (3D) stereoscopic pelvis model on first-year medical students learning of 3D relationships. Before its use, the pelvis model was modified based on basic science and clinical expert opinions regarding important structures for inclusion.

Methods First-year medical students $(n=37)$ participated in one of two learning sessions. One group $(n=25)$ attended a virtual 3D stereoscopic pelvis model presentation while another group $(n=12)$ attended a 2Dformatted presentation of pelvic anatomy images. The 3D group was administered knowledge pre-tests and mental rotation tests (MRTs) before the 3D presentation and knowledge post-tests, second MRTs, and surveys assessing $3 \mathrm{D}$ perceptions after the $3 \mathrm{D}$ presentation. The $2 \mathrm{D}$ group was administered knowledge pre-tests before the 2D presentation followed by knowledge post-tests and surveys.

Results One-way ANOVAs will be used to compare test scores before and after $2 \mathrm{D}$ and $3 \mathrm{D}$ learning sessions and the knowledge post-test scores between 2D and 3D groups. The results will confirm whether 2D-image or virtual 3D stereoscopic pelvis model presentations are more effective in improving first-year medical students learning of anatomy.

Conclusions Virtual 3D stereoscopic models have the potential to improve students learning when used in addition to traditional anatomy education. This study will evaluate the impact of expert-guided model modifications in improving students' anatomy learning. Future studies should address the effectiveness of virtual 3D stereoscopic models of other complex anatomical structures on students learning of 3D relationships.

\section{TECHNOLOGY \& INNOVATION}

Poster: 603
Integration of a Massive Open Online Course on Clinical Nephrology in Different Educational Settings

Peter GM de Jong, Rene A Hendriks, Franka Luk ${ }^{1}$, Marlies EJ Reinders

${ }^{1}$ Leiden University Medical Center, Leiden, Netherlands

Purpose In 2016 LUMC launched a Massive Open Online Course (MOOC) on Kidney, Pancreas and Islet Transplantation for students and healthcare professionals. As opposed to on-campus education, this course delivers learning objectives exclusively online. The MOOC has been integrated in different educational settings.

Methods The MOOC is offered monthly to learners worldwide and demographic data of participants are obtained. Furthermore since the year 2016 the MOOC is integrated in two 2nd year Medical School courses by using parts of the MOOC to actually replace traditional classroom teaching, as well as by using only a few movies, the discussion forum and a clinical patient case assignment as additional optional materials. Finally, the complete MOOC is offered to students in the extracurricular Honors track and in the yearly international Summerschool, and to several universities around the world participating in our virtual exchange program. Results Over 10.000 learners from over 90 countries signed up for the MOOC. During the two on-campus courses the MOOC is offered to over 300 medical students per year. Students report back that the MOOC elements are an interesting addition to the face to face curriculum and that the online lectures and discussion forums are inspiring. Over half of the students explore to some extent other parts of the MOOC outside the instructed assignments. During the Honors Track and Summerschool, the students seem to be much more engaged with the online materials and the discussion forums than the 2 nd year students. In the international exchange program the MOOC is received well.

Conclusions The online resources in a MOOC can be used successfully in different settings of education. The high quality materials, interactivity and online discussions offer added value to traditional classroom teaching. Further research is needed to see how this integration of materials can be further optimized.

\section{TECHNOLOGY \& INNOVATION}

Poster: 604

Teaching Anatomy with Virtual Reality: Development of Spatial Perception and Analysis of Structures and Functions

Mildred Lopez, Jose Gerardo Carrillo, Juan Pablo Nigenda Alvarez, Ricardo Trevio Gonzalez, Jorge E. Valdez Garcia, Belinda del Carmen Carrion

${ }^{1}$ Tecnologico de Monterrey, School of Medicine and Health Sciences, Monterrey, Mexico

Purpose When performing medical procedures, physicians must rely on their mental representations to size complex internal structures that are not directly visible on the skin. In its formation, this knowledge is acquired through the study of two-dimensional images. Currently, virtual reality is revolutionizing the teaching-learning process due to its value to offer a practical experience, at low cost and easy to administer against the alternative of performing cadaveric practices. The objective of the study was to evaluate the impact of virtual reality for the development of understanding, perception and spatial description, as well as the analysis of structures and functions.

Methods The sample consisted of 88 medical students from the Morphophysiology course. For the analysis, mean and standard deviation were considered. Afterwards an ANOVA variance analysis was used. 
Results The results indicate that virtual reality had a positive impact in spatial location of the structures related to the clinical case, particularly in the signaling of intrathoracic structures.

Conclusion It is necessary that educators participate in the establishment and consolidation of standards for the promotion and implementation of virtual reality in medical education, in order to guarantee an experience that ensures the achievement of the learning objectives of students, residents and teachers.

\section{TECHNOLOGY \& INNOVATION}

Poster: 605

\section{Balancing Learning Technology and Costs: a Framework to Consider Video-Based Education}

Carl Gustaf S Axelsson ${ }^{1}$, Karen Buckley ${ }^{2}$, Ole-Petter Hamnvik ${ }^{3}$, Michael $\mathrm{G} \mathrm{Healy}^{1}$, Matthew ORourke ${ }^{2}$, Traci Wolbrink ${ }^{4}$, Roy Phitayakorn ${ }^{1}$

${ }^{1}$ Department of Surgery, Massachusetts General Hospital, Harvard Medical School and NEJM Group, Boston MA 02114 USA

${ }^{2}$ NEJM Group, Waltham MA 02451 USA

${ }^{3}$ Brigham and Womens Hospital, Harvard Medical School, Boston MA 02115 USA

${ }^{4}$ Boston Childrens Hospital, Harvard Medical School, Boston MA 02115 USA

Purpose Value-based care uses a variety of metrics to ensure that a new treatment is both effective and economical. In medical education, there is an influx of new video-based learning technologies and applications. However, there is a lack of financial frameworks to estimate the costs associated with introducing new technologies for educators and programmatic development.

Methods We performed a focused literature review to create a framework (REC Roles, Equipment and Consumables) for the evaluation of the production cost of the New England Journal of Medicine (NEJM) Quick Take videos available on a range of topics related to internal medicine.

Results The most expensive component of these video-based education modules is the time cost. The average time taken to produce each video was $40 \mathrm{~h}$. This time included research, script editing, meeting with the content expert, storyboarding, graphics development, audio recording and editing, animation, file processing, staging for review, making edits and redistributing, and creating thumbnails. The most significant costs were medical illustrator time (\$1500-2000 per video) and content expert time (\$150 per video). Some costs were minimized in our video production process due to favorable resource availability, minimal use of equipment/simulators due to the exclusive use of animated graphics, and minimal opportunity cost.

Conclusions Application of the REC framework demonstrates that total cost with focused learning topic-videos was strongly related to the length of each video. The REC-framework allows educators to better project resource needs. Further work will apply the framework in other video development settings (e.g. utilizing non-animated video components) and compare total costs per learner, outcome and over time when compared to traditional didactic methods.

\section{TECHNOLOGY \& INNOVATION}

Poster: 606

\section{Evaluating the Effectiveness of $360^{\circ}$ Videos in Promoting} Engagement in Medical Education

Neeral Shah ${ }^{1}$, Nathaniel Larson ${ }^{2}$, Vivian Chan $^{2}$
${ }^{1}$ Department of Medicine, University of Virginia, Charlottesville, USA ${ }^{2}$ University of Virginia, Charlottesville, USA

\section{Award Nominee}

Purpose As $360^{\circ}$ video technology has become more advanced and accessible, new ideas regarding its utilization in medical training have emerged. With this study, we have sought to examine whether $360^{\circ}$ videos promote increased engagement over standard $2 \mathrm{D}$ videos among medical students.

Methods We enrolled 39 fourth-year medical students to watch two 4minute videos of exercises in an anatomy lab in a $360^{\circ} 3 \mathrm{D}$ format with an immersive headset or in 2D format on a laptop computer. Every two minutes, students were asked if they were thinking about any thoughts unrelated to the video and to rate their engagement on a scale of 0-100. Following the videos, participants ranked on a scale from 0-100 their concordance with 14 statements related to engagement, practicality, and interest in the technology. Participants were also asked to describe what they perceived as the positives and negatives of their video experience. Results While watching the videos, the average engagement reported by the $360^{\circ}$ video group was higher at each time point than the engagement reported by the $2 \mathrm{D}$ group. Further, the engagement remained high in the $360^{\circ}$ group through the 6 and 8 minute timepoints. In the post-video survey, the $360^{\circ}$ group reported a statistically significantly higher average engagement in seven out of eight of the relevant statements. $360^{\circ}$ video was rated as more practical and interesting than $2 \mathrm{D}$. There was no significant difference in the perceived ease of learning. Additionally, when asked to give feedback, more individuals in the $360^{\circ}$ group cited feeling engaged or involved.

Conclusions Overall, our results suggest that use of $360^{\circ}$ video may improve engagement for short videos used in medical education.

\section{TECHNOLOGY \& INNOVATION}

Poster: 607

Enhancing Anatomy Learning with Self-guided, Independent Digital Anatomy Table Activities

Aftab Merchant, Akiva Katz, Kayeromi Gomez

${ }^{1}$ University of Illinois College of Medicine - Rockford, USA

Purpose With more emphasis on clinical education in the health sciences curriculum, there is an increasing trend toward technology-enhanced educational resources for pre-clinical subjects, including Anatomy. However; mere acquisition of technology is unlikely to change the outcomes if the technology is not wisely selected and used. Educational technology resources, such as Digital Anatomy (Anatomage) Table, are great tools for students to master complex body structures; however, the adaptation of such technology within classrooms has been a considerable challenge so far. Instructors and students alike, all face a steep learning curve familiarizing themselves to the basic functioning of the table making it difficult to incorporate such systems into the curriculum. The purpose of this study is to address the challenges faced to include Digital Anatomy Table to enhance anatomy learning at a large medical school system with three campuses.

Methods To address the foremost challenge of training students and faculty to best use the Digital Anatomy Table in a limited time, we wrote a self-guided training exercise for the table that instructs users to complete certain tasks on the table and by doing so, they learn the most significant features of the table in a relatively short time (less than twenty minutes). The class size, the time required to complete the exercise, and the limited number of digital tables available, were considered during the development of the training module. 
Results The self-guided independent training will enable students with no or limited prior experience in the subject of Anatomy or on the digital table to identify, explore, and learn from simple to more complex anatomical structures independently.

Conclusion Our innovative approach would help many medical schools confronting the challenges of training faculty and students to use the Digital Anatomy Table efficiently to learn anatomy.

\section{TECHNOLOGY \& INNOVATION}

Poster: 608

\section{Game Design for Complex Skills: Using 4c/Id to Redesign a Game for Emergency Care}

\author{
Tjitske Faber ${ }^{1}$, Mary Dankbaar ${ }^{1}$, Jeroen van Merrienboer ${ }^{2}$ \\ ${ }^{1}$ Erasmus MCRotterdam, Netherlands \\ ${ }^{2}$ School of Health Professions Education, Maastricht University, \\ Maastricht, Netherlands
}

Purpose The ABCDE method, used internationally to treat seriously ill patients, is commonly trained in face-to-face-courses. When preparing for such a course with a game (abcdeSIM), training results improved for residents 1 . However, students playing the game did not outperform students studying identical text-based cases 2. Four-Component Instructional Design 3 (4C/ID) provides a framework for developing instruction for complex tasks. 4C/ID has been recommended for medical education and has been used in designing serious games in several fields. We have not encountered games for medicine designed using 4C/ID. We hope to inspire teams developing games for medicine on how to use $4 \mathrm{C} /$ ID in their design process.

Methods In a collaboration between content experts, game designers, and educationalists, we used 4C/ID to redesign abcdeSIM. We created additional learning tasks to ensure variation, increasing complexity and decreasing support within levels. Supportive and procedural information was added

Results Level of complexity was based on severity of disease and interventions required. Time-based prompts for vital interventions gradually decrease. A pause function enables access to supportive information. To assist overall problem-solving strategy development, a form prompts structured ABCDE assessment. For procedural information, we presented tool information upon selection and showed hit areas projected on the virtual patient to aid tool use.

Conclusions 4C/ID provides a structured approach for design choices in a serious game. Working in an existing game, little screen space was available for prompts and information. Designing the level of support was difficult because challenge is important to maintain flow. Timing the prompts to align with individual players needs remains challenging. By following 4C/ID principles and closely collaborating, we were able to build a new game version incorporating the theoretically sound support options described above.

References Dankbaar et al. Simul Healthc. 2016;12(1):9-16.

Dankbaar et al. Adv Health Sci Educ Theory Pract. 2016;21(3):505-521.

Merrienboer\&Kirschner. Ten Steps to Complex Learning. Routledge; 2017.

\section{TECHNOLOGY \& INNOVATION}

Poster: 609

Use of 3D Printed Models of Bone Tumors to Augment Medical Education

Shayna Youman ${ }^{1}$, Evan Dang ${ }^{1}$, Myers Jones ${ }^{2}$, Bonnie Brenseke ${ }^{3}$
${ }^{1}$ OMS-II Campbell University Jerry M. Wallace School of Osteopathic Medicine, Lillington, USA

${ }^{2}$ MSBS-2 Campbell University Jerry M. Wallace School of Osteopathic Medicine, Lillington, USA

${ }^{3}$ Department of Pathology, Campbell University Jerry M. Wallace School of Osteopathic Medicine, Lillington, USA

Purpose The purpose of this study is to create models of benign and malignant bone tumors from computed tomography (CT) and magnetic resonance imaging (MRI) scans and assess the effectiveness of the use of such models in medical education.

Methods CT and MRI scans of bone pathology were anonymized using 3D slicer software and imported into a free online converter for conversion into 3D digital models for printing. Pre-created digital models from a database were also printed. Two different 3D printing techniques were used: (1) Ultimaker 3 Extended printer using fused-deposition modeling (FDM) with colorFabb nGen white and clear filaments and (2) Form 2 printer using stereolithography (SLA) with Formlabs white and clear resins. Tinkercad was then used to apply geometric solids to bridge structural deficits of the models. The prints required trimming excess material and support structures. The SLA prints required additional processing steps: sit in two baths of isopropyl alcohol for 10 minutes each, dry off, and then cure under concentrated UV light for 15 to 30 minutes. Medical student volunteers were randomized into two groups (approved, IRB\#407). Both completed pre- and post-tests for understanding of bone pathology following a short lecture, one group with $3 \mathrm{D}$ printed models during the lecture and the other without. Results Printed models were able to imitate gross characteristics of the tumors and were produced at no cost to the research team. The impact on student education (results of pre- and post-tests) is still pending.

Conclusion $3 \mathrm{D}$ printing stylized models of bone tumors is a cost and timeeffective means to augment medical student understanding of this pathology. This study highlights the benefits of interprofessional practice and education as medical students had to work alongside engineering students to devise and execute the project.

\section{TECHNOLOGY \& INNOVATION}

Poster: 610

\section{Academic Medicine Student Interest Group: a Solution for Student} Curiosity

Herschel Wilde, Julie Anne Jahp, Skylar Larsen, Sarah Nguyen

${ }^{1}$ University of Utah School of Medicine, Salt Lake City UT, 84112

Purpose The student authors at the University of Utah School of Medicine (UUSOM) conceived of this innovation after identifying a lack of resources to learn more about careers in academic medicine (AM). Despite being embedded in an AM environment while in medical school, there are limited resource to learn about career paths, how to become involved, and how to prepare for a career in AM.

Methods The student authors and two faculty mentors created the Academic Medicine Student Interest Group (AMSIG) as a centralized resource for medical students to learn about, prepare for, and become involved with AM. AMSIG leadership is composed of three second year medical students, one first year medical student and two MD faculty advisors. This team plans events, facilitates mentorship, collaborates with administration and directs the future of the group.

Results AMSIG held two successful events and will hold a third during its first year. The events were well received by students and involved faculty alike. The three events were planned sequentially to first introduce career paths within AM, second to connect interested students with passionate faculty mentors, and third to help students develop skills to prepare for successful careers in AM. 
Conclusions AMSIG bridges a perceived resource gap at UUSOM by providing a centralized forum for medical students to learn about potential career paths, establish mentor relationships, and develop useful skills in the field of AM.

\section{TECHNOLOGY \& INNOVATION}

Poster: 611

\section{Dashboarding Data: Tools for Visualizing Faculty Engagement and Project Management}

\author{
Katherine Henderson \\ ${ }^{1}$ Virginia Commonwealth University, School of Medicine, Richmond, \\ USA
}

Purpose To highlight easy-to-use tools that collect, organize, and represent data associated with academic engagement, portfolios, and facultyrelated programming. Visual representations of data, or dashboards, can express narratives of success that can be captivating and persuasive (Vaitsis, et. al., 2014). There are several tools that can assist with this expression and faculty/faculty developers can use these tools to measure program effectiveness and/or quantify work for promotion and tenure. Learning to use select tools that help manage data and reduce information overload can address the challenge of low digital fluency among faculty, and help develop skill sets that are desired of medical educators (Johnson, L., et. al., 2014).

Methods This presentation will share three projects developed across departments in VCU School of Medicine. Specifically, work related to faculty engagement, process management, and data visualization will be discussed, emphasizing how each tool has improved tracking, reporting, and real-time analysis for decision making.

Results Data collected and presented with these tools, whether it be for P\&T or departmental programming, can be used to identify strengths, weaknesses, opportunities and concerns. Additionally, the creation of automated processes can save faculty/faculty developers time as they express narratives of success using data. A guide for how each of these tools can be implemented in a variety of contexts at other institutions will be provided.

Conclusions These tools are accessible from any device at minimal or no cost. Moreover, they do not require advanced skills in computer programming or knowledge of complicated syntax. However, a basic understanding of HTML can complement how a users' data can be presented. Many faculty may not have these skill sets; however, with a desire to experiment, learning to develop dashboards is feasible, and one can generalize this skill to a variety of contexts.

\section{TECHNOLOGY \& INNOVATION}

Poster: 612

The Use of Virtual Reality with Clinical Case Studies to Facilitate Learning of 3-Dimensional Anatomical Concepts in Cardiology

Roberto Galvez, Laura Shackelford, Jenny Amos, Judith L Rowen

${ }^{1}$ Carle Illinois College of MedicineUniversity of Illinois at UrbanaChampaign, Champaign IL, 61820 USA

\section{Award Nominee}

Purpose There has been recent interest towards utilizing virtual reality (VR) as an educational tool. As with any new tool it is important to determine what educational content VR is most effective in delivering. We describe a clinical case-centered VR task that was used to effectively teach first-year medical students the 3-dimensional aspect of collateral circulation, an anatomical property that is often difficult to teach with traditional methods.

Methods Groups of four students were given access to computers running the VR version of Organon (a commercially available anatomy program). Students were provided an overview of the software and then presented with a clinical case of a patient exhibiting symptoms consistent with aortic coarctation. They then completed the following tasks: (1) propose the most likely cause for the patients symptoms; (2) using the VR tools, diagram the most likely route for blood flow around the occlusion if the coarctation was (A) preductal or (B) postductal. Upon completion, each group submitted a single document with their proposed answers. Students also received further examination on this content in weekly quizzes (NBME-style, from Firecracker).

Results All 32 students completed the tasks to a sufficient level of competency within the 1 hour time. Weekly quiz performance indicated student competency; performance on 3 related questions was $97 \%$ for Carle Illinois students, compared to $76 \%$ for other US students. Upon examination of the activity as a whole, it (1) successfully educated the students on the predetermined objectives, (2) stressed a collaborative teamwork approach to learning, and (3) utilized VR to effectively assist with the educational objective.

Conclusion As medical schools move towards utilizing newer technologies, it is important to remain cognizant of what educational content is best conveyed with these technologies. We provide an example of educational content that was successfully provided through VR.

\section{TECHNOLOGY \& INNOVATION}

Poster: 613

Relationship Between Medical Student Engagement in an Immune Response Simulation Innovative Session and Examination Performance in a First-year Medical Microbiology and Immunology Class

Nyla Dil

${ }^{1}$ University of Central Florida College of Medicine, Orlando, USA

Purpose Undergraduate medical education is moving past traditional lecture based pedagogy and is shifting gears towards engaging students in active learning. Immunology is a complex discipline to comprehend and most entering medical students have little to no prior knowledge of this intricate discipline. We used a novel hands-on immune response simulation session to engage students in active learning of immunological principles and improve integration with medical microbiology. We developed tools to access student engagement during this novel active learning session and studied relationship between student engagement and examination performance. This abstract describes this medical education project and its outcomes.

Methods Students are taught didactic immunology lectures followed by this active learning session where students simulate an immune response scenario to major classes of pathogens. Students prepare $10 \mathrm{~min}$ long enactments playing roles of a host immune cell or a pathogen. Faculty facilitates this learning session and administers the assessment tools. Three integrated aspects of student engagements: cognitive, behavioral, and affective are measured. Performance was based on a multiple-choice questions final examination.

Results Preliminary results indicate that there is a significant increase in immunology knowledge gained and that students were highly engaged in learning immunology through this innovative pedagogy. There was a positive relationship between student engagement and final exam performance.

Conclusion Immune response simulation is an effective pedagogical novelty where students are actively engaged in learning complex 
immunological principles in a hands on experiential learning session. This novel pedagogical intervention might prove valuable in engaging students in other medical science disciplines as well.

\section{TECHNOLOGY \& INNOVATION}

Poster: 614

\section{Evaluation of Musculoskeletal Teaching in the Medical School} Curriculum

Arundathi Jayatilleke

${ }^{1}$ Drexel University College of Medicine, Philadelphia, USA

Purpose A solid understanding of musculoskeletal (MSK) disorders is essential for medical school graduates. Yet studies have shown students and graduates to be underprepared in MSK medicine. A potential gap thus exists between existing curricula and the knowledge required to provide excellent patient care.

Methods Evaluations from our institutions preclinical courses from 20132018 were reviewed for data about MSK exam and lecture sessions using keywords (joint/joints, MSK, and musculoskeletal). In addition, 3rd year shelf exam questions from 2016-2017 pertaining to MSK medicine were reviewed and compared to national means.

Results Quantitative feedback was available for 2016-2017. 52\% of 58 second year students responded that they felt confident in their ability to perform a MSK exam, $29 \%$ were neutral, $17 \%$ disagreed, and $2 \%$ strongly disagreed. In contrast, $86 \%$ of respondents felt confident in their ability to take a history and $95 \%$ in their ability to perform a general physical exam. 54 narrative feedback comments were analyzed to yield 63 data points in 11 areas: 32 comments made regarding MSK lectures and 31 regarding physical exam. In both areas, the most common comment was regarding timing of sessions. The second most common comment was that more MSK sessions were needed (6/32 lecture-related and 9/32 exam-related). Only one student requested less time in MSK lectures and no students requested less time in MSK exam teaching. 233 NBME clinical questions pertaining to the MSK system were assessed in the areas of foundational content (107), diagnosis (60), management (62), and prevention (4). Compared to national means, average scores were generally within 5 percentage points.

Conclusions Evaluation of our institutions preclinical course feedback suggests that students are not confident in their MSK exam performance and would also prefer further instruction in the field. Due to limited instructional time, novel methods of supplementing MSK instruction should be explored.

\section{TECHNOLOGY \& INNOVATION}

Poster: 616

\section{Game-Based Learning in Musculoskeletal Medicine}

Arundathi Jayatilleke

${ }^{1}$ Drexel University College of Medicine, Philadelphia, USA

Purpose Despite increasing interest, the use of games in medical education remains the exception rather than the rule. However, games can help students bridge the divide between preclinical education and clinical practice because learning is not only relevant but applied and practiced within the context of the game: i.e., situated cognition. Because of the need for increased musculoskeletal education of medical students, I am developing a digital game using an interactive patient scenario to teach elements of the musculoskeletal exam and related clinical reasoning to preclinical medical students.
Process In implementing backwards design to this intervention, I propose that students who have completed the game should be able to appropriately manage a patient presenting with subacute elbow pain and swelling. During the game, they will practice differentiating inflammatory, mechanical, and degenerative conditions affecting the elbow using historical clues, view and perform (virtually) an upper extremity musculoskeletal exam, and justify the appropriate next step in management based on their proposed diagnosis.

Implementation Second year medical students who have had instruction in both musculoskeletal pathology and physical exam will have access to the game as a supplement to the established curriculum. Outcomes will be assessed via performance on school and standardized examinations, as well as through a new clinical skills assessment case in the third year (preimplementation data is being collected).

Conclusion Games can provide an opportunity for situated learning, an especially important area for preclinical students exploring the role of practitioner. In this innovation, I am developing a game that fills a need (increased training in musculoskeletal exam) in a field underrepresented in preclinical education (rheumatology). Clinical skills and reasoning will be assessed before and after the intervention using a standardized patient encounter.

\section{TECHNOLOGY \& INNOVATION}

Poster: 618

Perceived Value of the Innovation Week: Developing Competen`es on Multidisciplinary Teams

\section{'Silvia Olivares, Miriam Turrubiates Corolla, Belinda Carrion}

${ }^{1}$ Tecnologico de Monterrey, Escuela de Medicina y Ciencias de la SaludAve. Morones Prieto 3000, 64710 Monterrey, N.L., Mexico

Purpose Tecnologico de Monterrey includes competency based education as a principle for its academic programs, including the School of Medicine and Health Sciences (EMCS for its acronym in Spanish). The I Week was designed to develop disciplinary and generic competences from challenging activities on a full immersion week. The aim of this study was to measure the perceived value of the I Week program considering the learned generic competences from students perspective.

Methods The study had a quantitative, transactional and descriptive design. Participants were 148 students from different programs (45\% from EMCS and 54\% from non-health related programs). Some examples of activities were related with: clinical simulation, lab research and anatomy art, among others. The pedagogical objectives were obtained from the online platform, and the perceived learning results were obtained from satisfaction survey (Cronbach Alpha 0.94).

Results The three generic competences more referred by the students of the Medical Basic Sciences during the I Week activities were: collaboration, intellectual curiosity, innovation and critical thinking. The most popular competences selected by design were: critical thinking and innovation. Critical thinking was the most aligned competence between design and achievement. Is relevant to notice that in just one week is possible to promote generic competences on health sciences related topics. Conclusion A break on the calendar of the regular courses to have the I Week is an innovative initiative to develop competencies instead of isolated knowledge. The I Week activities promoted collaboration among students across disciplines. The selected challenges introduced motivation to critically think how to deal with actual problems on a nonstructured process.

\section{TECHNOLOGY \& INNOVATION}

Poster: 620

Gamification Supporting Pharmacology Education: Analysis of Purpose and Structure in a Preclinical Curriculum 
Laurel Gorman, Vananh Do

${ }^{1}$ UCF College of Medicine, Orlando, USA

Purpose Previous data from our institution showed preclinical medical students rate gamification highly for active pharmacology learning, but few studies have evaluated how games should be integrated into curricula. To share how gamification was used for pharmacology education in our curricular model, a study was performed to determine the following: (1) sequencing, (2) purpose, (3) structure and complexity, (4) recall mechanisms.

Methods Preclinical pharmacology activities designed as electronic games or gamified sessions were identified. Sessions were defined as gamified if they employed peer competition, engaging methods including an invitation to play, and a large number of interactive questions. For descriptive analysis, games were categorized by purpose, categorical vs mixed topic presentation, curricular location, organizational complexity, and types of recall and feedback.

Results Twenty-seven pharmacology activities met gamification criteria. All contained immediate feedback and some outcome-related reward. The most common classroom games employed Turning Point $($ team competitions while the most common electronic game used a Jeopardy $@$ format. The most common purposes were review (52\%), flipped classroom (48\%), and peer engagement (44\%). Most were integrated into second year modules (56\%), structured categorically (52\%), and employed recognition-retrieval questioning $(63 \%)$. Complexity structure varied with $48 \%$ using interspersed complexity presentation while $44 \%$ employed a linear simple to complex design.

Conclusions Curricular analysis supports that gamification is used throughout the preclinical curriculum to support active pharmacology learning with the main purposes being review, flipped classrooms, and peer engagement. In support of educational literature to maximize learning, the games provide feedback to clarify gaps, dispersed retrieval-based practice to enhance retention, and many use interspersed complexity, a design previously shown to enhance critical thinking $1,2,3$. Sharing this curricular model will inform others on how to integrate preclinical games into curricula for effective active learning.

\section{TECHNOLOGY \& INNOVATION}

\section{Poster: 622}

\section{Now Featuring: Course Trailers to Increase Student Participation in Pre-Clinical UME}

Katherine Henderson

${ }^{1}$ Virginia Commonwealth University, School of Medicine, Richmond, USA

Purpose To increase learner intrigue and participation using course trailers. These short, faculty-produced videos describe course objectives and set learner expectations, while captivating learners through creative expression. Course trailers are four minute videos that condense and creatively express a course syllabus. Use of these videos may have a significant impact on students that are disinterested and not attending classes. Like Coursera and Khan Academy, these trailers use media and techniques to appeal to student interests, and emphasize the relevance, value, and importance of course objectives.

Methods The course trailer was presented to students via an embedded video within a Google Form survey. This poster will present a narrative framework (Truell, 2018) used to create the trailer, and will include an overview, script, and tools used to produce these media. An example of a VCU School of Medicine course trailer will be also provided.

Results Results from surveys that assess student interest, motivation, and communication preferences will be shared. The survey results are currently being collected. This and course evaluation data will be used for comparison. The production of course trailers may lead to increased student participation in UME, and promote deeper faculty engagement with innovative technologies.

Conclusions As videos become more common and easier to create, the production of course trailers is another skill faculty can use to generate intrigue about topics, and clarify ambiguity for complex, pre-clinical courses. This innovation also addresses the millennial generation communication preferences (Kron, et. al, 2010). Faculty must feel comfortable with their voices and have the ability to convey scientific information with metaphors and examples to diverse audiences. The linchpin to this projects success has been the synergy of interest, enthusiasm, and creativity of one psychiatry faculty member to lead this effort and influence her colleagues.

\section{OTHER}

Poster: 700

\section{A Modern Approach to Medical Education: Collaborative Anatomy-} Pathology Project

Jessica Sohn, Bonnie Brenseke, Howard Reisner, Terence Mitchell, Bruce Newton

${ }^{1}$ Campbell University School of Osteopathic Medicine, Lillington, USA

Award Nominee

Purpose The Collaborative Anatomy-Pathology (CAP) Project provides medical students a deeper understanding of anatomy, histology, pathology, and clinical medicine via linking disease seen at gross and microscopic levels with clinical implications of the disease.

Methods First year medical students' document lesions found during gross anatomy dissection. Samples are collected and undergo routine histologic processing. The resulting glass slides are scanned to permit virtual microscopy. After extensive literature review, participating students construct scientific posters, under faculty guidance, for presentation at research symposia. Examples of poster topics have included hypertensive nephropathy, teratoma, prostatic adenocarcinoma, and porcelain gallbladder. After presenting, students complete a 10-question Likert survey to assess the impact of the project on their medical education.

Results The CAP Project has been conducted for the past five years. Twenty-eight students participated in the first year and 49, 77, 63, and 98 participated in subsequent years (315 in total). The majority of surveyed participants agreed or strongly agreed the program increased their interest in anatomy (96\%), pathology (86\%), and research $(78 \%)$. The participants also noted an increased ability to perform literature searches (76\%) and compose a poster suitable for presentation at a scientific session $(94 \%)$.

Conclusions Integration of the basic and clinical sciences is one of the primary goals of modern medical education. Outcomes showed students were more observant during their dissections as they looked for pathological processes; and they learned how to compose and present a scientific poster as well as how to use medical informatics to research various disease states. The CAP Project demonstrates that anatomic dissection can be part of an innovative and integrative teaching strategy in medical education. The low cost $(\sim 8 / \mathrm{H} \& \mathrm{E}$ slide), ease of preparation, and reliance on available material make this an attractive option for many professional programs. Examples will be shown.

\section{OTHER}

Poster: 701 
Evaluating Students Experience in Conceptually Coherent Basic Science and Clinical Learning

\author{
Lee Cheng Jie Irene ${ }^{1}$, Lai Siang Hui ${ }^{2}$ \\ ${ }^{1}$ Duke-NUS Medical School Singapore, Singapore City, Singapore \\ ${ }^{2}$ Duke-NUS Medical School, Sing Health, Singapore City, Singapore
}

Purpose Making learning meaningful and relevant for medical students in preclinical education is critical in providing necessary foundation for the acquisition of clinical knowledge. The minimal contextual learning of basic science knowledge was perceived as a common issue related to preclinical education. Leveraging on conceptual coherence framework which emphasizes the importance in creating relevant mental representation of clinical and basic science information, we underwent purposeful reorganization in one of the preclinical courses (Molecules, Cells and Tissues) to better integrate basic science content with clinical learning. Application questions in Team-Based Learning format were rewritten to accurately reflect the underpinnings of basic science content, particularly its molecular and cellular pathways. The facilitated-discussion that followed in Team-Based Learning revisited the key learning points in a clinically-orientated manner. We aimed to determine if the reorganization of the content and application questions grounded with conceptual coherence framework facilitates better learning experience.

Methods To describe students learning experience with the changes implemented, we used thematic analysis on the qualitative feedback received in the course evaluations from current and previous cohorts. Course rating on specific domain such as the perceived clinical relevance of the course was also collected based on Likert scale that ranged from 1 (Strongly disagree) to 5 (Strongly agree). The rating was compared against the previous cohort.

Results The thematic analysis of the qualitative feedback identified the lack of clinical correlation as the major theme that surfaced in the previous cohort. Importantly, such perception was not identified in the current cohort. Consistent with the well-perceived clinical relevance of the restructured course, rating on the clinical relevance also increased from an average of 3.91 (SD 0.67) to 4.12 (SD 0.57).

Conclusions Corroborating with the framework of conceptual coherence, providing explicit connection between basic science learning and clinical context also improved students learning experience in basic science content.

\section{OTHER}

Poster: 702

The Predictive Value of Affective and Cognitive Empathy on Stability of Osteopathic Medical Student Specialty Choice

\section{Katrina Gerbrand, Godwin Y. Dogbey, Bruce W. Newton}

${ }^{1}$ Campbell University School of Osteopathic Medicine, Lillington, USA

Purpose Physician empathy is essential for building patient trust. Changes in medical student empathy have been studied; but this study evaluates how student empathy may be associated with stability of specialty choice throughout medical school. In other words, if the empathy of a student changes, do they also select a residency which correlates with their empathy scores? Core specialties have higher empathy scores than non-core specialties.

Methods Data was obtained from The Balanced Emotional Empathy Scale (BEES; measuring affective empathy) and Jefferson Scale of Empathy (JSE; measuring cognitive empathy). Surveys were given at the beginning of each academic year (M1-M4) and at the end of year 4 to the graduating classes of 2017 and 2018. Student gender and specialty preference was also gathered. 229 students filled out the surveys; 114 men and 115 women.
Results M1 BEES scores were not significantly associated with core (Family and Internal Medicine, OB/GYN, Pediatrics and Psychology) or non-core specialty choice (e.g., Radiology, Surgery, Emergency Medicine; $p=0.943$ ). Students who selected their specialty during their M1 year, and did a residency in that specialty, had a significantly higher M2 BEES score than those selecting their specialty in their M3 year ( $p=$ 0.037). M1, M3, and M4 BEES scores were not significantly associated with the year students chose their eventual residency. There were no significant associations between any JSE score with the year students picked their specialties.

Conclusions Finding an association between empathy and specialty stability could aid admission committees and students in determining which specialty a medical student would most likely choose, regardless of their initial interest in a certain specialty. However, the data shows specialty stability was not associated with affective or cognitive empathy scores; e.g., students with higher empathy scores who switched from a core to a non-core specialty, did not have a commensurate drop in empathy scores.

\section{OTHER}

Poster: 703

Developing an Interprofessional Education (IPE) Ethics Curriculum Experience Across 5 Programs: Pharmacy, Osteopathic Medicine, Health Information Management, Nursing and Physical Therapy

\section{Elizabeth K McClain}

${ }^{1}$ William Carey College of Osteopathic Medicine, Hattiesburg, USA

Purpose Identify, develop and assess first steps of an Ethics IPE project using a logic model and backward design to meet multiple program accreditation standards.

Methods This longitudinal IPE Ethics curriculum project utilized a logic model approach (inputs, activities and outputs) for IPE program development. Activities included assessment of IPE needs/barriers across programs (1) Accreditation requirements, (2) Training/ Faculty Development, (3) Academic schedules across multiple programs, (4) Common Curriculum and activities. Three levels of outputs were (1) short-term outcomes, (2) intermediate outcomes, and (3) long-term outcomes. Backward design principles (results, evidence and instruction/learning experiences) guided the IPE curriculum development.

Results Inputs included institutional support and engagement (5 of 7) recruited programs. Activities included targeted needs assessment development/delivery, monthly IPE team meetings and proposal request for formal IPE training. Short-term outcomes: 3 of 5 programs identified program accreditation requirements and barriers due to different academic schedules. All programs identified perceived need for faculty development, Ethics as a feasible topic for IPE and a curriculum delivery preference of synchronous, simulation and asynchronous learning activities. Intermediate outcomes included Institution support of IPEC formal offsite training and initial development plan of IPE ethics curriculum proposal. Programs identified a list of common ethics topics extracted from the 5 program syllabi. The team implemented an ethics Homeless Simulation with 175 students across the programs with assessment perception of health, poverty and homelessness. Initial goals for long-term outcomes were identified.

Conclusion The logic model provided an effective structure and guided initial steps of the IPE Ethics project. Backward design directed active communication and engagement of faculty stakeholders in curriculum structure and topics and linked them to results, evidence and activities to meet accreditation across programs. It will be critical to continue use of the logic model to monitor continued development in implementation intermediate and long-term outcomes. 


\section{OTHER}

Poster: 704

\section{Identifying Healthcare Provider Accommodations for Adult Patients with Autism Spectrum Disorder}

\author{
Courtney Todd, Mary O. Dereski \\ ${ }^{1}$ Oakland University William Beaumont School of Medicine, Auburn \\ Hills, MI USA
}

Purpose Each year, 66,000 patients with Autism Spectrum disorder (ASD) turn 18. There is little information about the transition to an adult health care provider for these patients. This pilot project attempts to ascertain healthcare delivery preferences and potential accommodations implemented by physicians who treat adult ASD patients.

Methods Physicians who currently treat adult ASD patients were identified by contacting Family Medicine [FM], Internal Medicine, and Internal medicine/Pediatrics [Med/Ped] providers in Michigan. Participating physicians completed an in-person interview. Responses were qualitatively evaluated for common themes and similarities or differences in responses. Results Three physicians were interviewed (1 FM in a small private practice, $1 \mathrm{FM}$ in a large teaching hospital, and $1 \mathrm{Med} / \mathrm{Ped})$. All participants stated they did not have a current protocol for ASD patients; did not obtain information from the patients previous physician; or instruct their clinical staff to make accommodations for these patients. Participants indicated that identifying an adult ASD patient is difficult, may happen by chance and there is no tool to bring awareness that they are treating an adult ASD patient. The large practice FM physician indicated that they were not taught on how to intervene [when encountering an adult ASD patient]. All expressed interest in viewing a Continuing Medical Education module on the topic.

Conclusion This study identified the lack of a common approach to providing primary healthcare to adult ASD patients. It also indicated that an accepted standard of care is absent for providing primary care to an adult ASD patient. These findings underscore the need for medical education programs presenting best healthcare practices for this growing adult patient population.

\section{OTHER}

Poster: 705

\section{A Quantitative and Qualitative Evaluation of Medial Pharmacology Textbooks}

Brian J Piper

${ }^{1}$ Geisinger Commonwealth School of Medicine, Scranton, USA

Purpose Goodman and Gilmans The Pharmacological Basis of Therapeutics (PBT) has a distinguished history and is a cornerstone textbook in the training of physicians, pharmacists, and pharmacologists. Similarly, Katzungs Basic and Clinical Pharmacology (BCP) is widely employed in biomedical education. As new editions were recently (2018) published, the goal of this project was to conduct an assessment of these influential educational resources. A prior report determined that only $11.2 \%$ of PBT, 12th edition and $17.1 \%$ of BCP, 13th edition authors were female (Piper et al. 2015, 2018). This report examined quantitative (author sex, recency of citations) and qualitative (expert reviews) characteristics of these textbooks.

Methods Qualitative evaluations were conducted by forming a panel of content experts (e.g. pharmacists, pharmacologists, physiologists, microbiologists, bioinformaticists). Experts assessed the strengths and limitations of content (1-4 chapters each) in their area of expertise. Quantitative characteristics obtained included sex of the chapter authors, and the recency of chapter and section citations for PBT $(N=3,576)$ versus BCP $(N=1,777)$. Analyses were completed with Systat with variability expressed as the SEM. RESULTS One-fifth of authors were female (PBT $=20.9 \%, \mathrm{BCP}$ $=17.0 \%)$. PBT citations were over a decade old (11.5 years) which was $12.3 \%$ older than BCP $(10.4, \mathrm{t}(5,321)=4.42, p<.0005)$. Neuropharmacology section citations $(13.8+0.4)$ were significantly less recent than gastrointestinal $(7.2+0.5)$ in PBT. Similarly, Central $(11.7+$ $0.4)$ and Autonomic Nervous System $(11.9+0.8)$ sections were significantly older than Smooth Muscle $(9.4+0.6)$ and Chemotherapeutic $(9.3+0.2)$ references in BCP. Qualitative evaluations noted several improvements in PBT relative to the 12th edition and are ongoing.

Conclusion These textbooks will continue to be key educational resources. Potential improvements for future editions include greater representation of female authors and inclusion of more recent citations.

\section{OTHER}

Poster: 706

\section{Predictors of USMLE Step 2 CK Scores and Residency Choices}

Julia Chong, Hye Young Yoon, Sang Lee, W. Patrick Roche, Jaehwa Choi

\section{${ }^{1}$ Mercer University School of Medicine, Macon, USA}

Purpose It has been suggested that the performance on the United States Medical Licensing Examination (USMLE) Step 1 and Step 2 Clinical Knowledge (CK) greatly influences a medical students choice in specialty. The current study was designed for two purposes: to determine what factors were predictive of Step 2 CK scores and to investigate if there was an association between Step 2 CK scores and specialty choice.

Methods A voluntary survey was sent to the graduating class of 2019 medical students at Mercer University School of Medicine (MUSM) after they had received their Step 2 CK scores. Fifty out of 108 students responded to the survey. Pearson correlation coefficient was determined based on the survey responses. Results The strongest predictor of the Step 2 CK score was the Step 1 score $(r=0.83, p<0.0001)$. There was less correlation between the Step 2 CK score and the number of question bank test questions completed ( $r=$ $0.33, p=0.06)$, the number of days dedicated to studying $(r=0.14, p=$ $0.45)$, and the hours per day dedicated to studying $(r=0.16, p=0.38)$. Prior to our study, we hypothesized that the majority of the high achieving students will plan to become highly competitive specialists. Interestingly, $57 \%$ of the students who scored 250 and above in Step 2 CK hope to match in MUSMs mission compliant primary care while $43 \%$ hope to match in narrower and more competitive sub-specialties.

Conclusions In conclusion, the USMLE Step 1 score is the greatest predictor of the USMLE Step 2 CK score, which further supports and reinforces our previous study finding that a strong foundation in the basic sciences during the first two years of medical school is critical to the future success of a medical student.

\section{OTHER}

Poster: 707

Exploratory Study of Factors Influencing Choice of Medical School in a School Located on the US/Mexico Border

Naomi Lacy ${ }^{1}$, Hector Ramirez ${ }^{2}$

${ }^{1}$ Texas Tech University Health Sciences Center El PasoEl Paso, USA

${ }^{2}$ Baylor Scott \& WhiteDallas, USA

Purpose Potential medical students choose not only where to apply but which offers of admission to accept. Foster summarized the extant 
literature on how students choose which school to attend, based on studies outside the USA, into 5 domains: curriculum, reputation of school, personal contact, location, and facilities. It is unclear how or if these domains are relevant in the United States given the differences in the education systems. We question what domains are of influence in the U.S.A. and our area, particularly as we have a location focused mission. In this study, we explore the underlying factors in data from a school on the USA/Mexico border.

Methods Participants were medical school students (M1 through M4). We collected data using an anonymous survey administered online. Data consist of answers to 17 items about the school and its location, 3 demographic questions, and 1 item on offers of admission. We analyzed the data using principal axis factor analysis with a promax rotation. A post hoc analysis looked at scale reliability for the resulting factors.

Results One hundred thirteen of approximately 400 eligible students chose to participate. Analysis resulted in the extraction of 4 factors. Based on the items in the factors and the results of the post hoc scale reliability analysis, we interpret these 4 factors as (1) educational \& campus climate, (2) social \& interpersonal, (3) culture of location, and (4) curriculum. Items expected to load as location are dispersed among these four factors. Cronbach's alpha for the resulting scales ranges from .63 to .79 .

Conclusion Item loadings are not consistent with the grouping categories identified by Foster. In particular, elements consistent with location are dispersed across our factors. A broader study is needed to determine if these factors hold in a larger sample across multiple US medical schools.

\section{OTHER}

Poster: 708

\section{Assessing Gender Differences in Influential Factors and Career Goals for Fourth-Year Medical Students who Choose Interventional Radiology}

Rebecca F. Y. Minner, Linda Deloney, Clinton Gauss, Mollie Meek

${ }^{1}$ University of Arkansas for Medical Sciences, Little Rock, AR 72205 USA

\section{Award Nominee}

Purpose Women remain underrepresented in interventional radiology (IR) with women comprising only $13.3 \%$ of IR residents and $9.2 \%$ of IR academic faculty. The purpose of this study is to better understand how influential factors differ between men and women who choose IR residencies, as well as gather data on the intended career paths of men and women choosing IR as a career.

Methods The IRB-approved study uses de-identified 2015-2017 Graduation Questionnaire data from the Association of American Medical Colleges national survey of fourth-year medical students. The data were collected during the transitional time from the IR fellowship training model to the IR residency training model. A focused analysis was on women $(n=118)$ and men $(n=483)$ choosing IR. These respondents were compared regarding factors that influence their decision to choose a specialty and regarding intended career path plans using a chi-squared test or Fishers exact test, as appropriate.

Results Women were more likely than men to indicate that mentoring was a strong influence $(p=0.0010)$, whereas men were more likely to indicate that their future family plans were a strong influence $(p=0.0047)$. Women were more likely than men to indicated future participation in research $(p=0.0496)$, teaching $(p=0.0402)$ and public health $(p=$ $0.0335)$ during their career.

Conclusion To combat the IR gender gap, there should be an increased effort by IR physicians to encourage and facilitate mentoring relationships with medical students. Since, women are more likely to want to enter into research, teaching, and public health settings, it is important to utilize this research to better recruit female medical students into IR and guide students to resources that allow them to achieve their desired career goals.

\section{OTHER}

Poster: 709

The Effect of Providing 2P Safety Walk Rally Activity on Benefit and Satisfaction Among Clinical Medical Students

Pornpimon Kasemsook, Thipsumon Tangsriwong, Thipsuda Tangsriwong, Orawan Chaiyamahapruk

${ }^{1}$ Buddhachinaraj Hospital Medical Education Center, Phitsanulok, Thailand

Purpose According to WHO, patient safety is significant and assigned in doctor of medicine program in many countries including Thailand. In our medical school, Patient safety is not only taken seriously but also Personal safety as well (2P), the patient will be safe if a healthcare provider is safe. In order to encourage both patient and personal safety, 2P safety walk rally activity was provided for medical students. The study's aim was to evaluate the medical students benefit and satisfaction of this activity.

Methods 2P safety, for example needle stick injury and blood exposure prevention, infection control and medication safety, were integrated into the walk rally activity, an active and fun team building activity with a mixture of competitive team games in a set format, by multidisciplinary staffs. In extracurricular hours, the 4th-6th year medical students from Buddhachinaraj hospital were divided into small groups, academic year separately. Different topics were used in different academic year. Each group attended five activities with limited time and competed with others. 5-point Likert scale of activity's benefit and satisfaction questionnaires were completed by the students at the end of the session.

Results The questionnaires were completed by 185 students (response rate was 97\%). Hand hygiene procedure, high alert drug usage and effective communication were the most beneficial patient safety activities for 4 th, 5th and 6th year medical students respectively. The most beneficial of 4th and 5th year students' personal safety procedures were educational risk management and needle stick injury prevention. Overall were satisfied of this activity (4.03/5).

Conclusions Most medical students were benefited and satisfied from this activity. As the students learn better in a positive learning environment, the walk rally activity is one of effective learning processes which facilitates direct purposeful experience in our medical students while they are having fun.

\section{OTHER}

Poster: 710

\section{Stressors and Coping Mechanisms of Medical Students}

Mariah Rudd, David Musick, Brock Mutcheson, Aubrey Knight

${ }^{1}$ Virginia Tech Carilion School of Medicine, Roanoke, USA

Purpose Research has documented significant levels of psychological distress and burnout in medical students. Some research has found that medical school can erode the students' natural resilience to stress. The goal of this project is to identify self-reported coping mechanisms, compare them with the stressors and determine how the medical students are caring for themselves and present opportunities for the students, faculty and staff to provide timely and critical interventions. 
Methods A two-part survey was administered to all students during spring semesters of Academic Years 2015-2016, 2016-2017, and 2017-2018. Students were provided with an informed consent form prior to taking. The survey instrument was comprised of two validated instruments, the "COPE Scale" and "Perceived Stress Scale." Data was handled by an honest broker and was de-identified. Qualitative themes were derived from the open ended response question. Item means were compared using the T-test procedure, with a significance level of $p=.05$.

Results A total of 242 responses of Virginia Tech Carilion School of Medicine students were collected using the brief electronic survey. Statistically significant changes were identified for four items. Students were asked to identify three events/experiences that have caused stress, responses themes include research expectations, personal/family, step exams, and residency selection/match process.

Conclusion Using a survey comprised of validated instruments to measure stress and coping, the authors were able to identify medical students key stress experiences and also identify the key positive coping mechanisms they utilize. For this longitudinal study, the same survey tool will again be given electronically to all medical students in 2019 and 2020. As this is a five-year study, future analyses will investigate relationships between stress levels, coping mechanisms, and performance. We will also further investigate the trends across education status and cohort.

\section{OTHER}

Poster: 71

\section{Evaluation of a Gender Gap in the Academic Performance of an Integrated Hybrid Curriculum}

Amy E. Medlock, Amy Baldwin, DeLoris Wenzel Hesse, Lia Bruner, Edwin V. Sperr, Jr., Janette R. Hill

${ }^{1}$ AU/UGA Medical Partnership and University of Georgia, Athens, USA

Purpose Studies have shown that gender differences exist for performance on both the MCAT and the USMLE Step 1 exam. Data show that men outperform women even when controlling for prematriculation factors. In addition, other studies have also documented gender differences in academic performance within specific curricula. This gap has been reported to narrow or even reverse throughout UME with women outperforming men on the USMLE Step 2 CK exam. A proposed cause for the gender gap in basic science content performance is curricular structure, specifically the integration (or lack thereof) of basic and clinical science materials. Thus, we hypothesize that students in an integrated hybrid curriculum may have a gender gap initially, but that this gap would disappear or reverse over the curriculum. Methods To determine if a gender gap exists in academic performance on our campus, we compared summative assessment of groups of men and women for the first two years of our four-year UME curriculum. These formative assessments included anatomy exams and module final exams during the basic science years. Future analysis will include clerkship shelf exams and NBME practice exams.

Results Initial analysis of summative assessment data from anatomy exams and module final exams show a gender gap does exist, with men outperforming women for year 1 of the curriculum. Preliminary analysis suggests that this gap may decrease from year 1 to year 2 . Further analysis of multiple classes, as well as standardized exams, is now underway. Conclusion Preliminary analysis has suggested a gender gap in academic performance early within our curriculum, thus warranting further investigation. We will expand our preliminary data analysis at our campus, compare with other curricula, and begin to investigate causes for gender gaps within UME.

\section{OTHER}

Poster: 712
Factors Associated with Osteopathic Medical Students Academic Perfor`mance

\section{Khalil Eldeeb, Godwin Dogbey}

${ }^{1}$ School of Osteopathic Medicine Campbell University, Lillington, USA

Purpose Selecting the most suitable applicants into medical schools has received much attention in recent times. Over the past decades, considerable research has examined the relationship between pre-admission criteria and academic success. However, these findings have been inconclusive. This study seeks to examine the pre-admission variables that are associated with medical student academic performance.

Methods This IRB approved study is a retrospective review of admission and academic performance records of the first-two graduated student cohorts from the Campbell University Jerry M. Wallace School of Osteopathic Medicine in North Carolina. Summary measures frequencies/percentages for categorical variables and means, standard deviation/range for continuous variables will be generated. Measures of association between the pre-matriculation variables and each set of postmatriculation outcome (performance) variables will be calculated using 2tailed Pearson product-moment correlation coefficients or Fisher exact test. Univariate, multivariable, and multivariate statistical analyses will be performed as relevant and necessary. All statistical inferences will be considered significant whenever, $P 0.05$.

Results (Preliminary) Entering science GPA and MCAT scores (total, biology and physical components) are positively correlated with first and second year medical school GPAs as well as COMLEX-1 total score. The verbal component of the MCAT positively correlated with total COMLEX-1 score only. Entering non-science GPA was positively correlated with year 2 GPA while overall entering GPA was similarly correlated with medical school years 1 and 2 GPAs but not with COMLEX-1. Years 1 and 2 GPAs were highly positively correlated with each and moderately with total COMLEX-1 score.

Conclusion Pre-admission academic factors are associated with matriculation academic and first-level national board examinations performance of osteopathic medical students. Admission processes should consider the importance of these factors in student recruitment to maximize the success of students.

\section{OTHER}

Poster: 713

\section{Adapting to Educational Challenges in Delivering Academic} Programmes in the Gulf

\section{Davinder Sandhu}

${ }^{1}$ Royal College of Surgeons in Ireland, Medical University Bahrain, Adilya, Bahrain

Purpose Bahrain is a small island near Saudi Arabia with a population of $1.2 \mathrm{~m}$ people. A key priority for Royal College of Surgeons in Ireland, Bahrain (founded, 2004) was to develop the School of Postgraduate Studies and Research established in 2009. This involved creating a culture of research in the university. Setting up the research infrastructure and new programmes, and the research strategy. This study looks at learning outcomes from delivering the research strategy in Bahrain/GCC.

Methods There are no external grants, and to overcome this, annual $\$ 100,000$ internal grant funds were provided competitively to faculty and $\$ 100,000$ capital grants. Grant holders must involve and supervise students. Regular research methodology courses for students. Joint research fund of $\$ 50,000$ created with Arabian Gulf University (AGU) to develop collaboration. Masters in Healthcare Management (2007), 
Quality and Safety in Healthcare (2008) and Nursing (2009) created base for postgraduate programmes. In 2015 , five $\mathrm{PhD}$ scholarships $(\$ 250,000$ each) allowed joint research with RCSI Dublin. Online publications supported. Research assistants appointed to cope with capacity to conduct research.

Results The Management and Nursing Masters successful with 411 and 80 graduates conferred since inception. Dissertations built sense of research responsibility and presentations. Research with AGU allowed RCSI to utilize their animal house. Since 2015, thirteen Symposia held. Student summer research projects expanded in 2018, to sixty students and 24 projects. Since 2010, ten faculty have achieved PhD or Doctoral programmes. 48 publications achieved in 2017. Good uptake by basic scientists but difficult to involve hospital and community doctors.

Conclusion Important to understand local Arabic culture, Ministerial regulations and participate in national committees. Establish good relations with neighbouring universities. Hosted programmes can be a vehicle for development of academic programmes. Support the University with grants and $\mathrm{PhD}$ programmes. Difficult to establish Masters programme in other disciplines due to small population. Constant conflict between teaching load and undertaking research. Much rhetoric, repetitive data submission and quality assurance visits and bureaucracy about implementing research.

\section{OTHER}

Poster: 714

\section{Physician Assistant Faculty Advisement During Creation of an Ultrasound Student Interest Group}

Ian M. Smith, Sarah J. Garvick

${ }^{1}$ Wake Forest School of Medicine Dept of PA StudiesWinston-Salem, USA

\section{Award Nominee}

Purpose Use of ultrasound in medicine is becoming a necessary skill set. However, the intensive training required for advanced competency in ultrasound is prohibitive for most medical curricula. One method for students to gain advanced training is an optional student interest group. This abstract gives recommendations for successful advisement during initial implementation of a Physician Assistant (PA) Ultrasound Student Interest Group (USIG) at an institution with dual campuses.

Methods All students are introduced to medical ultrasound early in the curriculum, as they receive instructions on basic utilization of ultrasound and techniques for evaluation. A subset of students advocated for the creation of a USIG desiring additional training. Although the group is student-led, faculty with clinical experience in medical ultrasound have been essential in the development. Faculty involvement included alignment of educational goals, oversight of bylaw creation, guidance for institutional protocols, and preservation of the USIG over time. Furthermore, having dual campuses required close faculty support to guarantee continuity of content through lectures from subject matter experts, workshops, and labs at each program location.

Results Sixty out of 89 students $(67.4 \%)$ participated in the inaugural year. Participants from both campuses $(n=20$ and $n=40)$ demonstrated an interest in increasing their ultrasound skills. Students participated in 8 workshops during their first year of PA education. Topics included a focus on point of care ultrasound (POCUS) skills, among others. Workshops were advertised to other medical learners throughout the institution to encourage interprofessional education and collaboration.

Conclusion Establishment of a USIG is vital for students who want to accelerate their training in medical ultrasound for clinical practice. Faculty advisement is imperative to ensure design of a valuable and successful resource that allows for a relevant, goal oriented experience that is sustainable for PA students.

\section{OTHER}

Poster: 715

\section{A Student Centered Approach to Medical Education Through Learning Communities}

LaToya Sherman, Zsa-Zsa Booker

${ }^{1}$ Wayne State University School of Medicine, Detroit, USA

Purpose On account of the urban medical school having more than 300 students per cohort and 1,200 students per academic year, the ability to provide pervasive enhanced student learning and engagement opportunities was finite. An advisory group was developed in order to create a learning community program that fostered a sense of community service, mentor-ship, scholarship, social engagement, and health and wellness. This presentation describes the evolution of learning communities.

Methods Over the last several years, faculty, professional staff, students, and the Vice-Dean of Medical Education convened to meet bi-weekly to plan, design, and implement specified goals at an advisory group capacity. Themes addressed were why learning communities, learning community structure, and learning community funding. The Advisory Group discovered that learning communities provide positive student learning outcomes due to its overarching principles of providing longitudinal relationships with faculty and students (Osterberg et al, 2016).

Results Eight new learning communities were created with 36-42 students per community. Social - Student engagement has improved by the addition of more than 16 new student led social activities. Community Service - 51 community service projects were completed. Mentorship Learning communities are comprised of students and 2-3 faculty and student mentors per group. Health and Wellness - Each learning communities hosted a health and wellness seminar. Scholarship - Students participated in the Community Impact Day Program that involved research and work with patients who experienced one of eight different health disparities.

Conclusion Students deemed learning communities to be invaluable to the educational experience and are committed to their improvement. Considering that learning communities are student centered, the communities have caused more collaboration and teamwork, and has also created a more diverse learning environment for physicians in training.

\section{OTHER}

Poster: 716

Physicians Perspective on their Preclinical Medical School Curriculum

Judith Binstock $^{1}$, Maria A. Pino ${ }^{2}$

${ }^{1}$ Touro College of Osteopathic Medicine, New York, USA

${ }^{2}$ NYIT College of Osteopathic Medicine, Glen Head, USA

Award Nominee

Purpose This preliminary study is the first to ask physicians how they would change their medical school preclinical curriculum for bestpractice medicine. The purpose of this research project was to answer (1) Did osteopathic physicians consider their preclinical curriculum supportive of best-practice medicine? (2) What suggestions would these physicians have to improve their preclinical curriculum?

Methods A 12-question survey was emailed to the 2011-2018 alumni of Touro College of Osteopathic Medicine, Harlem, NY. Questions were asked on demographics and type of practice, but focused on the assessment of specific basic science courses by asking should have more, 
sufficient or should have less. Additional questions included interest in having clinically relevant basic science presentations and the importance of including other topics, e.g. basic statistics, early patient exposure, clinical cases, etc. A statistical analysis (SPSS) was performed and the relationships between variables was tested using a Pearson chi-square $(a=$ $.05)$.

Results (1) Demographics: The respondents $(N=122)$ were $55 \%$ male and $44 \%$ female; most were practicing or associated with an academic hospital $(57 \%)$ and $48 \%$ were primary care while $52 \%$ were non-primary care physicians (including Emergency Medicine); (2) Basic Science Courses: Pharmacology (41\%), Pathology (39\%) and Physiology (39\%) ranked the highest as Should have More while Histology/Lab (45\%) and Embryology (35.5\%) ranked the highest as Should have Less; (3) 60\% were interested in clinically relevant basic science presentations; (4) Early patient exposure (65\%), increasing clinical cases (70\%) and analysis of journal articles $(70 \%)$ were ranked highest as Should have More. A statistical analysis between variables and questions regarding preclinical courses found little significant differences and no overall patterns.

Conclusion Pharmacology, Pathology and Physiology were identified as should have more as were clinical cases, journal article analysis and early patient exposure. Asking practicing physicians what they feel would help them in best-practice medicine is crucial in designing future medical school curriculums.

\section{OTHER}

Poster: 717

\section{Strength in Numbers: Using Group Peer Review for Grant Review}

Mariah Rudd, David Musick

${ }^{1}$ Virginia Tech Carilion School of Medicine, Roanoke, USA

Purpose The expertise of multiple reviewers is often sought for the review of manuscript, grant and other scholarly submissions for purposes of quality control and assurance. The peer-review process, a commonly independently driven activity, has recently been flipped upside down with the advent of Group Peer Review. Group review relies on the complementary expertise of a small team, requires the active exchange of ideas, and necessitates ongoing collaboration. A team of health professions educators recently deployed the Group Peer Review process for the review of national grant submission.

Methods A study team member was asked to independently review a group of proposals for a national grant. Instead, a group of several experts was recruited to independently provide a score and comments for each grant proposal. The individual scores were averaged and comments from each reviewer were distributed amongst the group. The group came together to summarize, discuss, dissect, and share thoughts on each proposal. The group came to a consensus for a recommendation.

Results Anecdotal feedback from members of the grant review team demonstrated the value of participating in a group peer review exercise. Contributions from all members of the group resulted in an aggregate score for each proposal as well as robust feedback. The process served as a meaningful faculty development exercise for all reviewers who ranged in levels of experience with the peer review process.

Conclusions By bringing together individuals with different levels of experience and unique but complementary areas of expertise, the review process evolves into a less siloed, more holistic, faculty development opportunity with the end result of a more thorough and quality review.

\section{OTHER}

Poster: 718

Continuing Medical Education for Primary Healthcare Providers Treating Young Adults with Autism

Megan McCrohan, Mary Dereski

${ }^{1}$ Oakland University William Beaumont School of MedicineRochester, MI, 48309 USA

Award Nominee

Purpose National data indicates that 66,000 individuals with Autism Spectrum Disorder (ASD) will reach the age of adulthood annually; therefore, many primary care providers will likely treat ASD patients. Previous studies indicate that knowledge of ASD, along with appropriate accommodations, can improve healthcare delivery.

Methods A module was developed describing typical ASD behaviors, including clinical approaches to enhance the healthcare experience. The module was distributed to primary healthcare providers. Efficacy for increasing knowledge and awareness of ASD was assessed through preand post-module surveys.

Results Twenty-one primary care providers chose to enroll in the research study online ( $n=14$ Family Medicine, $n=5$ Internal medicine, with the remaining from Pediatrics, Internal Medicine/Pediatrics, and Preventative Medicine). Participant responses indicated that autism knowledge about the correct number of ASD individuals turning 18 annually, increased by $64 \%$ after taking the module. When asked How familiar are you with the challenges that patients on the Autism spectrum face when transitioning to adult primary care?, respondents increased their familiarity from $38 \%$ in the pre-survey to $100 \%$ in the post-survey. When asked if a patient or their previous physician has not disclosed that your new patient is on the Autism spectrum, would you ask the patient to fill out a form?, premodule responses increased from 50\% indicating definitely or yes to $92 \%$ for post-module responses. In the post-survey, $50 \%$ of participants indicated that they would definitely approach the delivery of healthcare to a patient with ASD differently, with $100 \%$ stating they would make accommodations after completing the module.

Conclusion This study suggests that a module providing ASD information and potential patient-related accommodations may increase healthcare provider awareness regarding how to improve healthcare delivery for this population. It may, therefore, be warranted for primary care providers to have access to this information prior to treating patients with ASD. 